University of Louisville

ThinkIR: The University of Louisville's Institutional Repository

Electronic Theses and Dissertations

8-2009

\title{
Exploring a new theory of successful aging among low-income older adults in an independent and assisted living community.
}

Valerie Lander McCarthy

University of Louisville

Follow this and additional works at: https://ir.library.louisville.edu/etd

\section{Recommended Citation}

McCarthy, Valerie Lander, "Exploring a new theory of successful aging among low-income older adults in an independent and assisted living community." (2009). Electronic Theses and Dissertations. Paper 934. https://doi.org/10.18297/etd/934

This Doctoral Dissertation is brought to you for free and open access by ThinkIR: The University of Louisville's Institutional Repository. It has been accepted for inclusion in Electronic Theses and Dissertations by an authorized administrator of ThinkIR: The University of Louisville's Institutional Repository. This title appears here courtesy of the author, who has retained all other copyrights. For more information, please contact thinkir@louisville.edu. 
EXPLORING A NEW THEORY OF SUCCESSFUL AGING AMONG LOW-INCOME

\title{
OLDER ADULTS IN AN INDEPENDENT AND ASSISTED LIVING COMMUNITY
}

\author{
by \\ Valerie Lander McCarthy \\ BSN Indiana University, 1999 \\ University of Louisville
}

\begin{abstract}
A Dissertation
Submitted to the Faculty of the

Graduate School of the University of Louisville in Partial Fulfillment of the Requirements

for the Degree of
\end{abstract}

Doctor of Philosophy

School of Nursing

University of Louisville

Louisville, Kentucky

August, 2009 



\title{
EXPLORING A NEW THEORY OF SUCCESSFUL AGING AMONG LOW-INCOME OLDER ADULTS IN AN INDEPENDENT AND ASSISTED LIVING COMMUNITY
}

\author{
By \\ Valerie Lander McCarthy \\ BSN Indiana University, 1999
}

\section{A Dissertation Approved on}

July 29,2009

by the following Dissertation Committee:

Disserthatiom Director

$\therefore \operatorname{sic}$ 


\section{DEDICATION}

This dissertation is dedicated to my husband, John, who encouraged me to pursue my dream and gave me unstinting love and support every step of the way.

John, thank you for your confidence in me, all the sacrifices you've made, and your endless patience in listening to me babble on about variables, statistical significance and IRB's, with a sweet smile on your face and only a slightly glazed look in your eyes.

I also dedicate this work to my daughters, Katie and Megan, and to my granddaughters, Anna and Abby, who gave me the constant joy in my life that helped me keep my dream alive through the hard times. 


\section{ACKNOWLEDGEMENTS}

I am grateful to so many people who have helped me make this journey. First and foremost, I want to thank Dr. Barbara Speck, my advisor and dissertation chair, for guiding and challenging me, from the day I applied to the doctoral program through the day I defended my dissertation. I thank you for your rigorous scholarship, your patient guidance, your ability to know when to urge me on and when to rein me in, and most importantly, for the hugs delivered precisely when most needed!

I thank my committee: Dr. Karen Robinson, who opened the world of aging policy to me; Dr. Celeste Shawler, for her collegiality and for remembering what being in dissertation is like; Dr. Joseph Petrosko, for guiding me through statistical analysis without injury to my pride; and Dr. Jane Thibault, for teaching me not only about aging, but about courage, grace, and spirituality in real life.

I owe a debt of gratitude to all of the faculty who helped me through my course work and development as a PhD student, especially Dr. Linda Holbrook Freeman and Dr. Kay T. Roberts. I also thank Sigma Theta Tau, the International Honor Society of Nursing, for dissertation support. Finally, I thank the dedicated staff and generous residents of Chapel House and Friendship House at Christian Care Community for allowing me entry to your very special community, and for sharing your wisdom and loving care with me. 


\begin{abstract}
EXPLORING A NEW THEORY OF SUCCESSFUL AGING AMONG LOW-INCOME OLDER ADULTS IN AN INDEPENDENT AND ASSISTED LIVING COMMUNITY
\end{abstract}

\author{
Valerie Lander McCarthy
}

July 29,2009

BACKGROUND: Current successful aging theory excludes individuals with disease or disability, limits the potential for successful aging in minority and lower socioeconomic populations, focuses on behavioral determinants of health rather than social and environmental determinants, and fails to consider older adults' own criteria for successful aging. A recent mid-range nursing theory (Flood, 2005) proposed that two factors -adaptation and gerotranscendence - are primary contributors to a new view of successful aging that may address these problems.

PURPOSE: The goal of the study was to investigate relationships among adaptation, transcendence, and successful aging to explore the potential of Flood's (2005) theory to serve as a guide to nursing research, practice, and policy.

METHODS: A cross-sectional survey study was conducted among a stratified, random sample $(\mathrm{N}=112)$ of non-demented older adults living independently or receiving assisted living services in an urban low-income continuing care retirement community. 
Investigator administered surveys were conducted with small groups ( $\mathrm{n}=5$ to 7$)$.

Recruitment minimized the number of surveys subsequently excluded due to dementia (6.4\%). Data collection, including consent, dementia screening, and administration of surveys was completed in a single session, while preserving participants' privacy and dignity. Efforts were made to accommodate sensory or literacy limitations.

RESULTS: The study found significant support for Flood's (2005) theory of successful aging. Adaptation and transcendence explained $45.4 \%$ of the variance in successful aging, controlling for age, income, function, and health. Transcendence accounted for two-and-a-half times the effect of adaptation. Ninety-two percent of participants reported they were aging successfully, regardless of age, health, function, income, or objective measures of successful aging. The study also supported the use of the newly developed Successful Aging Inventory (Flood, 2008) as an outcome measure to capture the new definition of successful aging (alpha .82).

CONCLUSIONS: Flood's theory of successful aging appears to merit further study of its potential to address the needs of older adults with disease, disability or socioeconomic disadvantages. Transcendence may be an important contributor to a holistic, positive view of aging that has not been previously reported in the successful aging literature. 
EXPLORING A NEW THEORY OF SUCCESSFUL AGING AMONG LOW-INCOME OLDER ADULTS IN AN INDEPENDENT AND ASSISTED LIVING COMMUNITY

\section{TABLE OF CONTENTS}

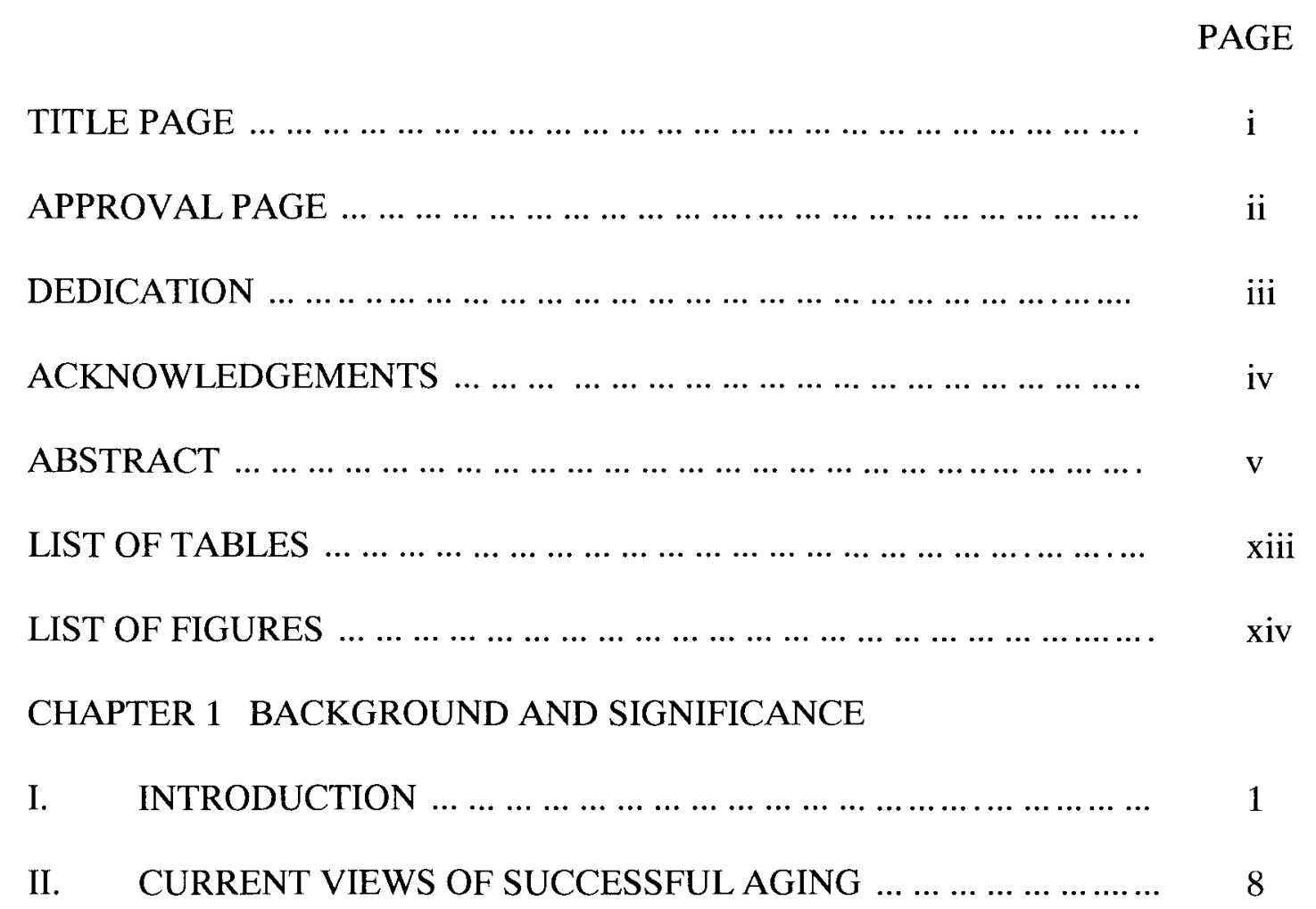

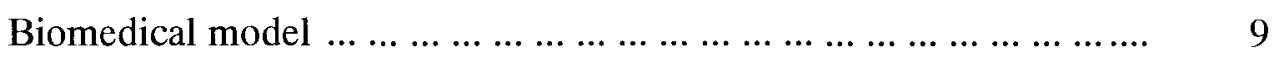

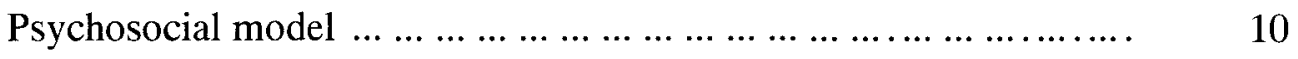

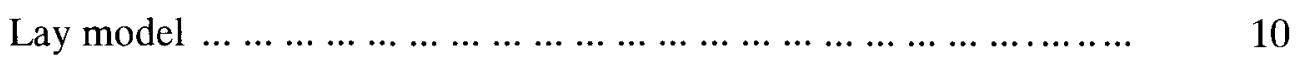

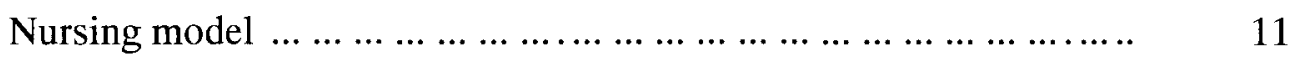




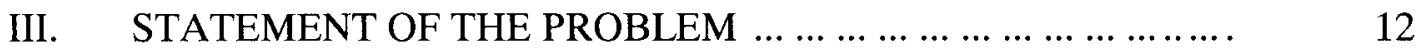

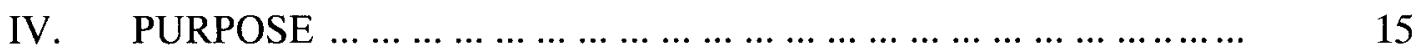

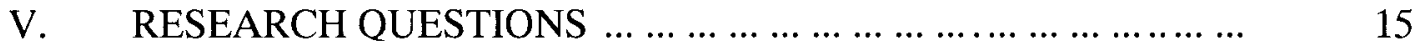

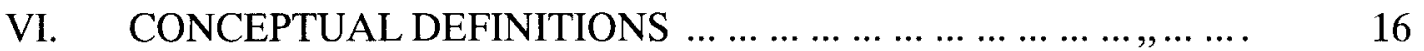

VII. DELIMITATIONS AND ASSUMPTIONS ................... 20

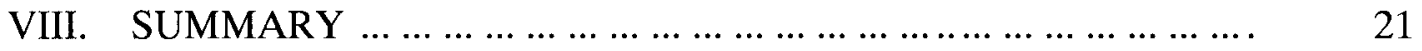

\section{CHAPTER 2 REVIEW OF THE LITERATURE}

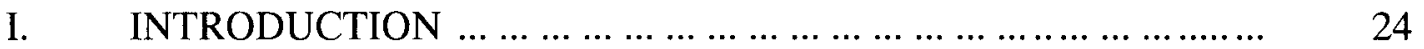

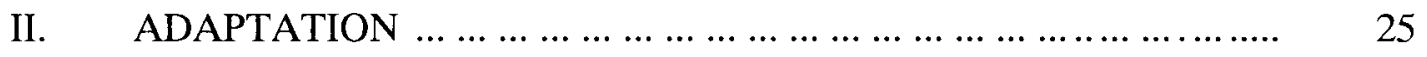

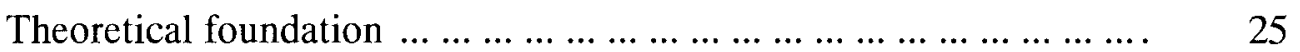

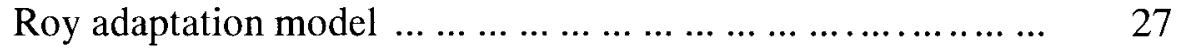

Selection, optimization, and compensation metamodel ........ 28

Proactive adaptation theory $\ldots \ldots \ldots \ldots \ldots \ldots \ldots \ldots \ldots \ldots . . \ldots \ldots \ldots$

Concept of proactive coping $\ldots \ldots \ldots \ldots \ldots \ldots \ldots \ldots \ldots \ldots \ldots \ldots \ldots \ldots \ldots$

Aspinwall and Taylor: Proactive coping theory .............. 33

Schwarzer and Taubert: Proactive coping theory $\ldots \ldots \ldots \ldots \ldots \ldots \quad 34$

Comparison of proactive coping conceptualizations ........... 36

Operational definition of adaptation: Proactive coping ............... 39

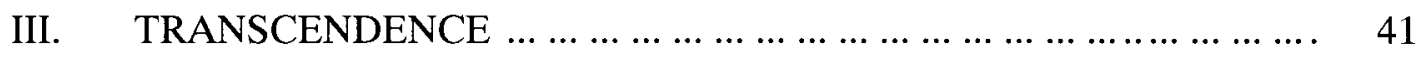

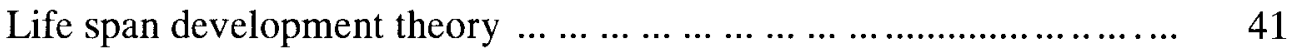

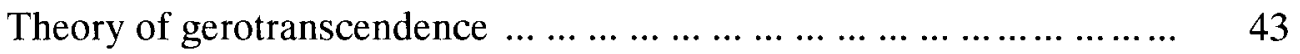

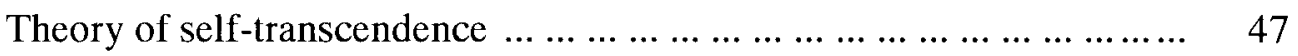

Operationalized definition of transcendence: Self-transcendence ....... 51 
PAGE

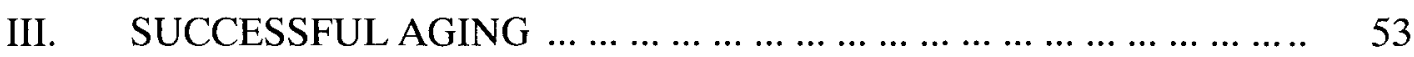

Biomedical perspective on successful aging $\ldots \ldots \ldots \ldots \ldots \ldots \ldots \ldots \ldots \quad 53$

Psychosocial perspective on successful aging $\ldots \ldots \ldots \ldots \ldots \ldots \ldots \ldots . \ldots \ldots$

Lay perspective on successful aging $\ldots \ldots \ldots \ldots \ldots \ldots \ldots \ldots \ldots \ldots \ldots . \ldots \ldots$

Summary of criteria for successful aging $\ldots \ldots \ldots \ldots \ldots \ldots \ldots \ldots \ldots \ldots \ldots . \ldots \ldots$

IV. MID-RANGE NURSING THEORY OF SUCCESSFUL AGING ..... 61

Description of the successful aging theory $\ldots \ldots \ldots \ldots \ldots \ldots \ldots \ldots \ldots \ldots . \ldots \ldots$

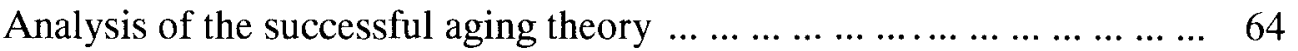

Evaluation of the successful aging theory $\ldots \ldots \ldots \ldots \ldots \ldots \ldots \ldots \ldots \ldots \ldots$

Operational definition of successful aging $\ldots \ldots \ldots \ldots \ldots \ldots \ldots \ldots \ldots \ldots \ldots$

V. $\quad$ FEASIBILITY STUDY $\ldots \ldots \ldots \ldots \ldots \ldots \ldots$

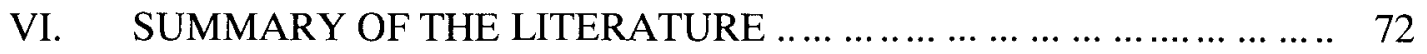

CHAPTER 3 METHODS

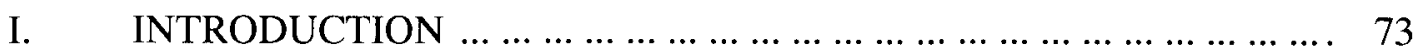

II. STUDY DESIGN AND PURPOSE $\ldots \ldots \ldots \ldots \ldots \ldots \ldots \ldots \ldots \ldots \ldots \ldots \ldots$

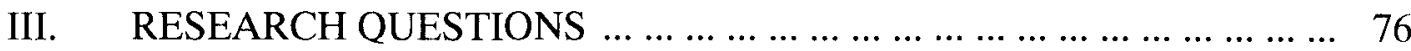

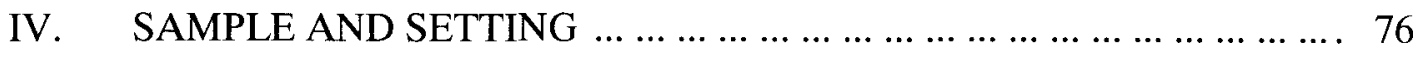

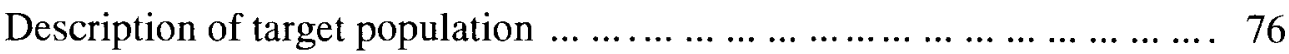

Setting $\ldots \ldots \ldots \ldots \ldots \ldots \ldots n$

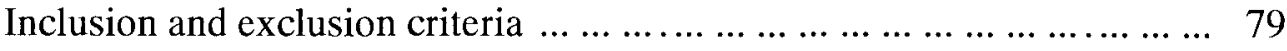

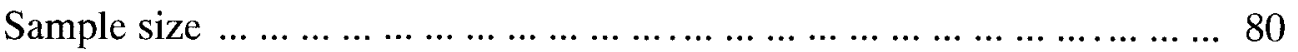




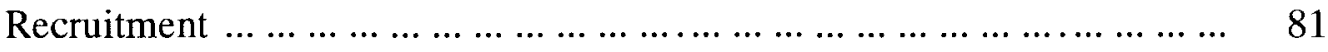

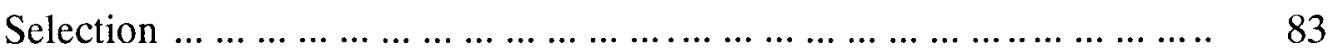

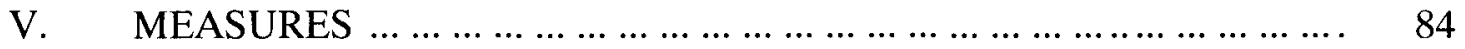

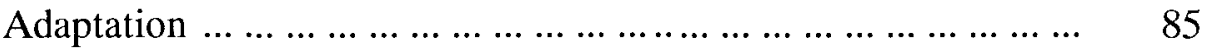

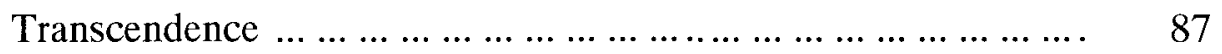

Successful aging $\ldots \ldots \ldots \ldots \ldots \ldots \ldots \ldots$

Investigator-developed questionnaire $\ldots \ldots \ldots \ldots \ldots \ldots \ldots \ldots \ldots . \ldots 1$

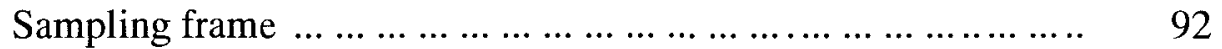

VI. DATA COLLECTION $\ldots \ldots \ldots \ldots \ldots \ldots$

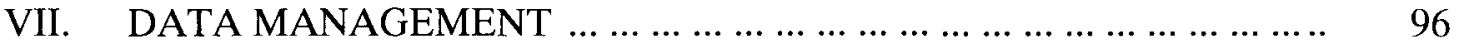

VIII. DATA ANALYSIS $\ldots \ldots \ldots \ldots \ldots \ldots$

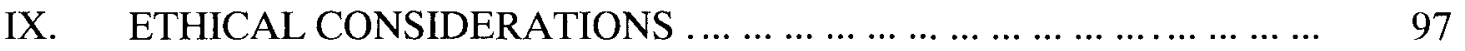

X. $\quad$ LIMITATIONS $\ldots \ldots \ldots \ldots \ldots \ldots$

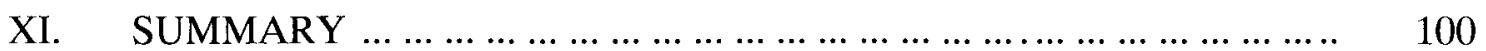

\section{CHAPTER 4 RESULTS}

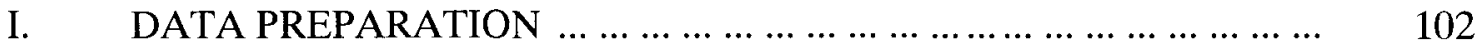

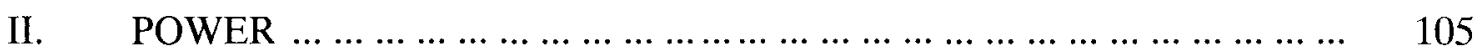

III. STATISTICAL TESTS AND ASSUMPTIONS $\ldots \ldots \ldots \ldots \ldots \ldots \ldots \ldots \ldots \ldots$

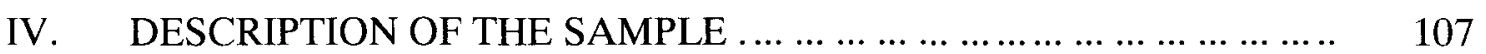

V. PAIRWISE CORRELATIONS AMONG RELATED VARIABLES ..... 109

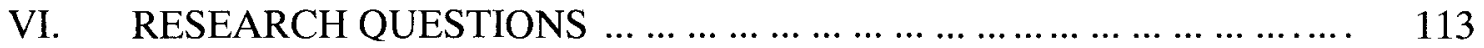




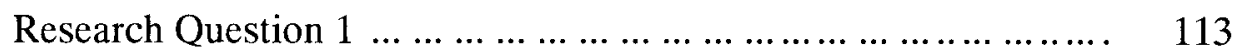

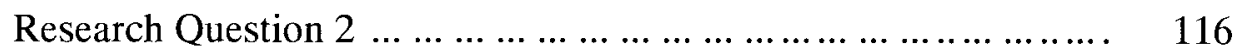

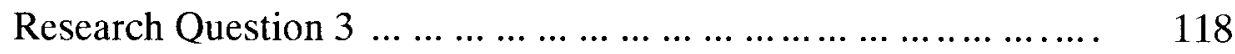

Research Question $4 \ldots \ldots \ldots \ldots \ldots \ldots \ldots \ldots \ldots \ldots \ldots \ldots \ldots \ldots . \ldots \ldots$

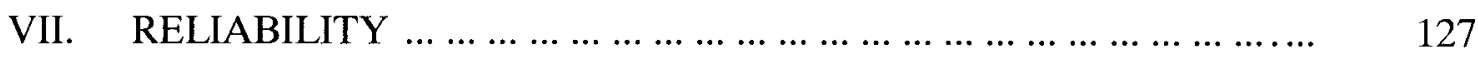

\section{CHAPTER 5 DISCUSSION}

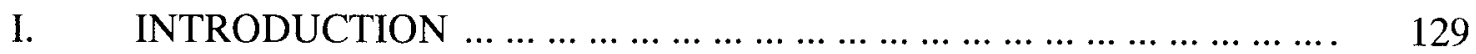

II. OVERVIEW OF THE STUDY $\ldots \ldots \ldots \ldots \ldots \ldots \ldots$

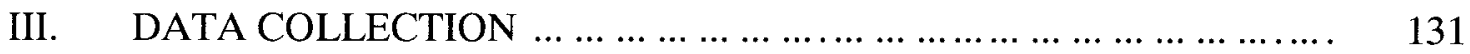

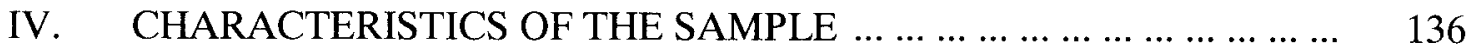

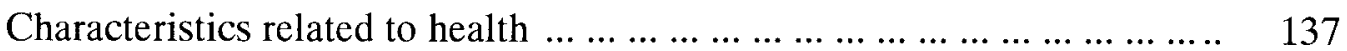

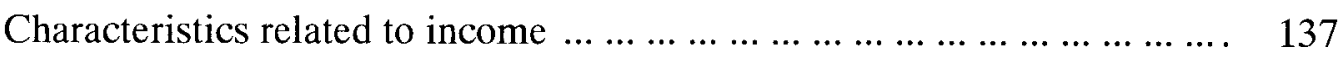

Characteristics related to function $\ldots \ldots \ldots \ldots \ldots \ldots$

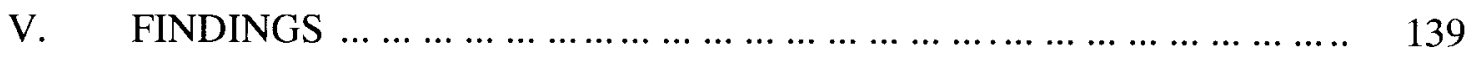

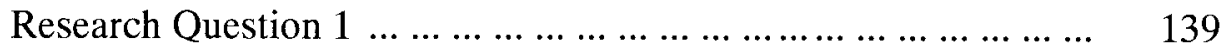

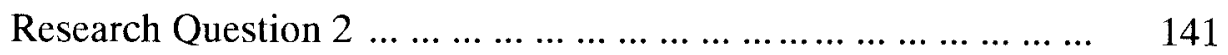

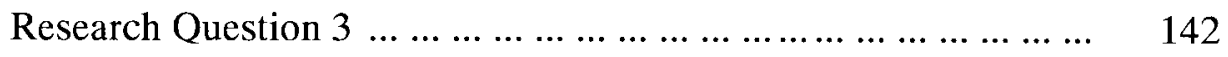

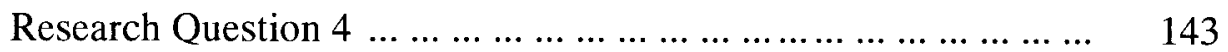

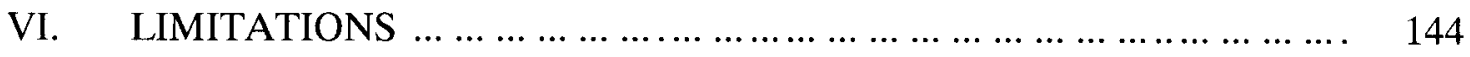

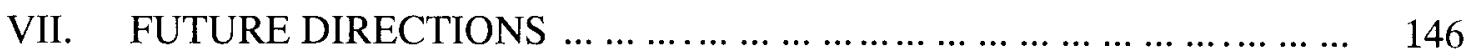

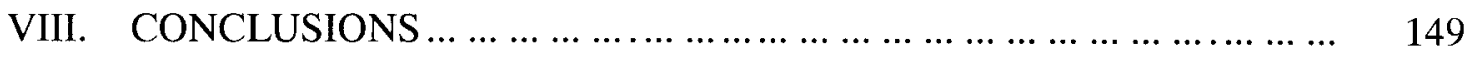

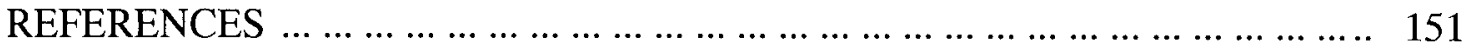


A. $\quad$ Mini-Cog dementia assessment test $\ldots \ldots \ldots \ldots \ldots \ldots \ldots \ldots \ldots \ldots \ldots \ldots \ldots$

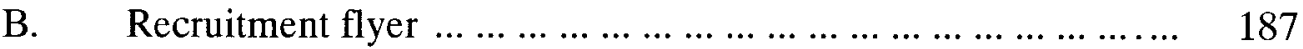

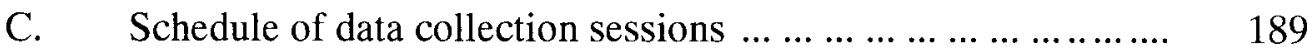

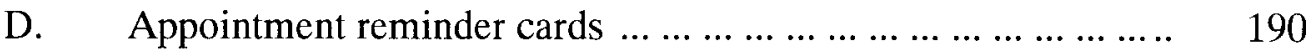

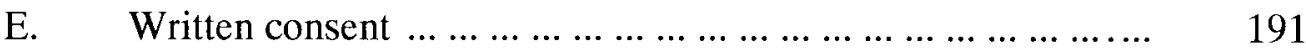

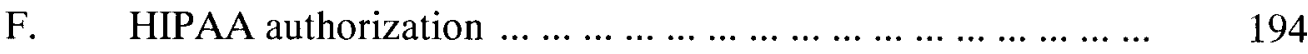

G. Proactive coping subscale of the PCI $\ldots \ldots \ldots \ldots \ldots \ldots \ldots \ldots \ldots \ldots \ldots$

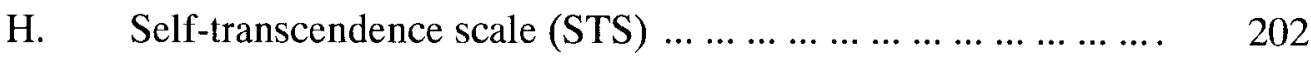

I. Successful aging inventory (SAI) $\ldots \ldots \ldots \ldots \ldots \ldots \ldots \ldots \ldots \ldots \ldots \ldots$

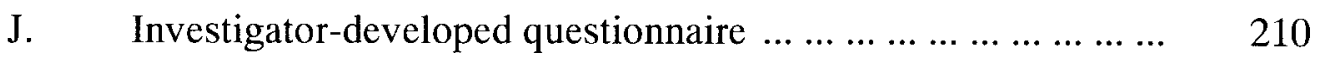

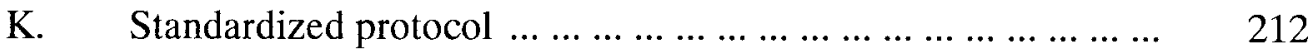

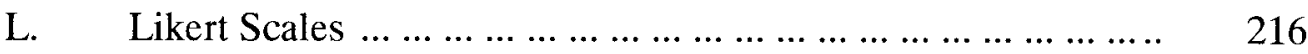

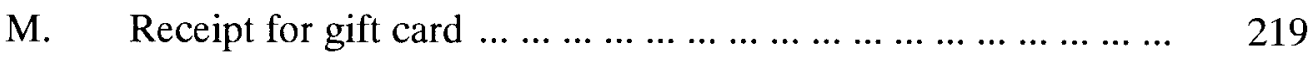

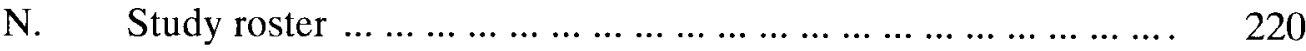

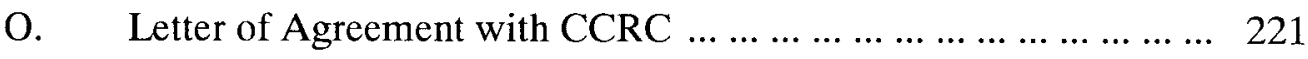

P. Institutional Review Board Approvals ........................ 222

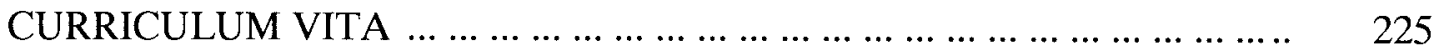




\section{EXPLORING A NEW THEORY OF SUCCESSFUL AGING AMONG LOW-INCOME OLDER ADULTS IN AN INDEPENDENT AND ASSISTED LIVING COMMUNITY}

\section{LIST OF TABLES}

TABLE

PAGE

Table 1 Conceptual and Operational Definitions for Study Variables .......... 17

Table 2 Characteristics of the Target Population $\ldots \ldots \ldots \ldots \ldots \ldots \ldots \ldots \ldots \ldots \ldots$

Table 3 Descriptive Statistics for Primary Variables ... ... ..................... 104

Table 4 Characteristics of the Sample Compared with the Population .......... 108

Table 5 Pairwise Correlations of Related Variables ....................... 110

Table 6 Bivariate Correlations among SAI, Sociodemographic

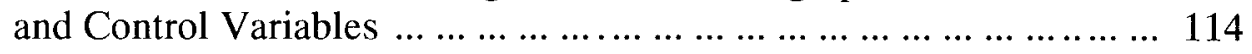

Table 7 Differences in Mean SAI for Sociodemographic

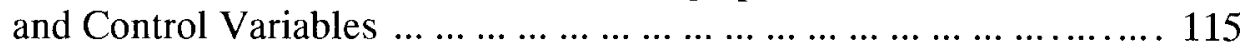

Table 8 Bivariate Correlations among SAI and Two Predictors $\ldots \ldots \ldots \ldots \ldots \ldots 117$

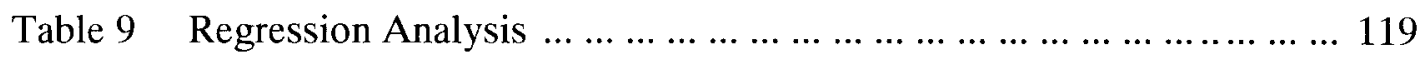

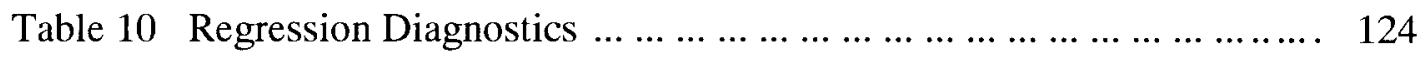

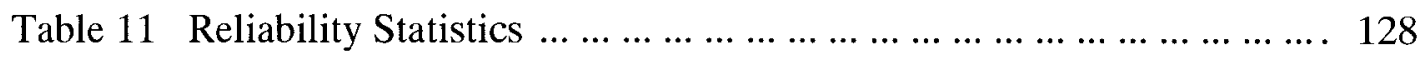


EXPLORING A NEW THEORY OF SUCCESSFUL AGING AMONG LOW-INCOME OLDER ADULTS IN AN INDEPENDENT AND ASSISTED LIVING COMMUNITY

\section{LIST OF FIGURES}

FIGURE

PAGE

Figure 1: Flood Model of Successful Aging Theory … .................. 13

Figure 2: Model of Relationships among Study Variables ................. 74

Figure 3: Plots Related to Regression Assumptions .................... 1.25 


\section{EXPLORING A NEW THEORY OF SUCCESSFUL AGING AMONG LOW-INCOME OLDER ADULTS IN AN INDEPENDENT AND ASSISTED LIVING COMMUNITY CHAPTER I \\ BACKGROUND AND SIGNIFICANCE}

Beginning in 2010, 78 million members of the "baby boom" birth cohort will enter their senior years, doubling the older adult population by 2030 (U.S. Census Bureau, 2006). Simultaneously, advances in public health and medical research have markedly increased longevity. At the turn of the $20^{\text {th }}$ century, life expectancy in the United States was about 45 years; 100 years later, at the turn of the $21^{\text {st }}$ century, it was 78 years (President's Council on Bioethics, 2002). The result is an unprecedented increase in the number of older adults in the U.S. population, with rapidly lengthening lifespans.

There is great variability among the older adult population; characteristics of a 65 year old are likely to be quite different from those of an 85-year old. A common categorization that provides greater clarity is young-old (age 60 - 69), old-old (70 - 79) and oldest-old (age 80 and older) (Garfein \& Herzog, 1995). The fastest growing segment of the current older adult population is among the oldest-old (Hobbs \& Stoops, 2002). While the young-old may remain relatively healthy, active, and independent, the significance of the increase in the numbers of oldest-old lies in the escalating frailty, 
increased incidence of chronic disease, risk of decreased cognitive function, and increased prevalence of disability in very late life (Baltes \& Smith, 2003).

The results are intensified needs for care and support at the same time that available resources may become exhausted. Especially among older adults with lowincome, racial and ethnic minorities, and among women - who outnumber men two to one in the oldest-old age group (U.S. Census, 2006) - there is a greater likelihood of outliving financial and social resources, subsequently living alone and in poverty in very old age (Hobbs \& Stoops, 2002). The arrival of the baby boom birth cohort will magnify the demands of the older adult population exponentially. By the year 2030, it is projected that $25 \%$ of the U.S. population will live with multiple chronic conditions at a cost expected to reach more than $\$ 1$ trillion (Anderson \& DeTurk, 2002). The rate of disability in very late life is projected to increase to more than $50 \%$ (Baltes \& Smith, 2003).

It has been suggested that projections extrapolated from current sociodemographics may not hold true for the baby boom birth cohort, who are expected to be healthier, wealthier, and better educated than today's older adults, although baby boomer's health behaviors are mixed; for instance, smoking has decreased, physical activity has remained unchanged, and obesity has increased (Knickman \& Snell, 2002). Nevertheless, the baby boom generation has redefined every life stage through which it has passed; it has flooded maternity wards, overcrowded classrooms, created a sexual revolution, and challenged the economy to absorb massive numbers of new workers (Whitbourne \& Willis, 2006). If the pattern holds, baby boomers may also redefine aging. 
Potential differences between the current older adult population and the upcoming baby boom generation are uncertain (Moody, 2008). What is certain is that there will be unprecedented numbers of older adults passing through late life within a relatively brief period of time, lending urgency to the need to address both the challenges and the opportunities of aging. To do so, two social factors that shape these challenges and opportunities must be considered: ageism and disparities among vulnerable populations.

As opposed to other "isms" based on race, gender, and class, ageism is the only stereotypical prejudice that is still socially acceptable (Butler, 1969; Nelson, 2004). Ageism is defined as discrimination based on age, especially prejudice against the elderly (American Heritage Dictionary, 2003). An examination of a display of greeting cards is one way to demonstrate this reality. The image of older adults as senescent, unattractive, and dependent is not only prevalent among younger people, causing elders to be stigmatized and marginalized, but has been internalized by elders themselves, decreasing self-worth and creating fear and apprehension about aging (Nelson, 2004).

The second social factor affecting the experience of aging involves disparities that exist among vulnerable populations, specifically women, racial or ethnic minorities, individuals with chronic disease and disabilities, or those with low socioeconomic status. Comparing the present view of aging to the Victorian era, which defined good health as the deserved result of living a life of virtue, while defining poor health as the just deserts of vice and self-indulgence, Holstein and Minkler (2003) made a cogent argument that gender, race, and socioeconomic status must be considered when assessing accountability for health and subsequent successful aging. Not only an individual's responsibility for health behaviors and lifestyle choices must be considered, but also an individual's 
"response-ability" (p. 792), that is the ability of an individual to make healthy choices.

Life experience, social conditions, and economic resources shape an individual's choices and behaviors, by influencing awareness of options that may exist, the individual's selfefficacy to achieve change, and the availability of resources.

Holstein and Minkler (2003) called attention to the current focus of aging research on behavioral determinants of health, e.g., lifestyle choices and behaviors made earlier in life that affect health and quality of life in old age, while noting there was not an equal focus on social and environmental determinants of health. The attitude that individuals control their own successful aging through lifestyle choices and behaviors is evidenced by a statement made by Rowe and Kahn (1998), stating the goal of the 10-year, $\$ 10$ million MacArthur Successful Aging Study was to identify the factors that "put one octogenarian on cross-country skis and another in a wheelchair" (p. xii). However, as Holstein and Minkler (p. 96) point out:

Rowe and Kahn's view doesn't ask if the 80-year-old skier had country club privileges and a winter home in Colorado, or the 80-year-old in the wheelchair had cleaned houses for a living while holding down a second job as a nurse's aide on the graveyard shift in a nursing home.

Schulz and Northridge (2004) examined interactions among social inequalities, the environments in which people live, and population health. The interaction of social determinants of health (income, education, cultural identity, discrimination, access to health care) with environmental determinants (crime, housing, nutrition, pollution, community services), according to Schulz and Northridge, directly affect the health and well-being of individuals and populations. Knickman and Snell (2002) argue that 
changing social, economic, and political policy is vital to improve health and thus decrease demands placed by aging baby boomers on society, especially by providing innovative community resources and services targeted to maintain functional ability and independence as long as possible.

A growing trend in the United States, combining a focus on social and environmental determinants of health with innovative community-based resources and services, is the increase in retirement housing and services. Although there is no consensus on terminology for the wide array of housing and service options for older adults (Eldercare.gov), for the purposes of this study an independent and assisted living retirement community refers to housing and services that accommodate elders' unique needs, encourage independence, avoid or delay placement in nursing homes, and improve quality of life (Hawes \& Phillips, 2007; Kane \& Wilson, 2007; Wilson, 2007; Zimmerman \& Sloane, 2007). While elders are reluctant to leave their family homes (AARP Public Policy Institute, 2008), research has strongly supported the importance of avoiding social isolation, lack of cognitive stimulation, and threats to safety inherent in remaining alone in a home as frailty and multiple chronic disease progress over time (Aud \& Conn, 2003; Cannuscio, Block, \& Kawachi, 2003; Cutler, 2007; Spitzer, Neuman, \& Holden, 2004; Stevens-Ratchford \& Diaz, 2003). Especially among lowincome elders living alone, physical and financial limitations make home maintenance an increasing problem, leading to the risk of substandard housing and an increased threat of falls and accidents (Hebeler, 2007).

Retirement housing and services, such as independent and assisted living retirement communities, when economically feasible, provide a safe and secure 
environment, improve nutrition, increase walkability and physical activity, and offer assistance with daily activities, thereby promoting health and encouraging independence (Nelson, 2008). Assistance with transportation enables maintenance of long-time friendships, church attendance, and volunteer activity (Fried, Freedman, Endres, \& Wasik, 1997). Planned social activities promote development of peer relationships, increasing functional and emotional social support, and providing opportunities for cognitive and sensory stimulation (Shu, Huang, \& Chen, 2003; Spitzer, Neumann, \& Holden, 2004; Stone \& Reinhard, 2007; Wilson, 2007; Zimmerman \& Sloane, 2007). When assisted living services are added to independent living, assistance with personal care, supervision of medications, and monitoring by trained staff may aid earlier identification of changes in function or behavior that might indicate new or worsening health conditions, delaying or preventing hospitalization or nursing home placement (Marek, Popejoy, Petroski, Mehr, Rantz, \& Lin, 2005). Independent and assisted living communities may be either freestanding, offering one or both options, or part of a continuing care retirement community (CCRC). A CCRC combines independent and assisted living with a skilled nursing facility, and sometimes other services such as an Alzheimer's care unit, within a single building or campus (Centers for Medicare \& Medicaid, 2007).

There are several sources of funding for retirement housing and independent living services, but funding for assisted living is less available. Funding sources include: (a) government-sponsored low-income housing for the aged and disabled; (b) non-profit retirement communities, supported by some combination of personal income, public support, and charitable organizations; and (c) proprietary for-profit retirement 
communities entirely funded by residents from private income, assets, family assistance, or long-term care insurance (SeniorHousing.Net, 2008). The third option, for-profit retirement communities, accounts for the fastest growing segment of the market, while funding for low-income independent and assisted living has remained flat or decreased (Heumann, 2004).

Limiting retirement housing and services to those older adults with sufficient financial resources exemplifies the need for increased attention to social and environmental determinants of health for all older adults. This need arises from concerns about the potential implications of demands - both current and anticipated - that will be made by an unprecedented number of older adults. Failure to effectively address the opportunities and challenges of aging for this changing demographic may diminish quality of life for older adults, their families, and caregivers. The well-being of society as a whole may also be impaired by overburdening health and social services, draining economic and intellectual resources, and limiting opportunities to pursue other priorities (Baltes \& Smith, 2003; Knickman \& Snell, 2002).

Knickman \& Snell (2002) suggested that the impact of the increasing number of elders might be moderated by promoting a more positive view of aging, addressing a full range of factors - behavioral, social, and environmental - that shape old age. A primary U.S. goal is to eliminate health disparities among low-income and minority populations (National Institute of Health (NIH), 2008). Another goal, both national and international, calls for adding life to years, not just years to life (Healthy People 2010, 2000; The United Nations, 2000; World Health Organization (WHO), 1999). A more comprehensive successful aging theory may help achieve these goals. 


\section{Current Views of Successful Aging}

A variety of theoretical viewpoints envision an old age that is positive, productive, and hopeful, including robust aging, (Garfein \& Herzog, 1995); healthy aging, (Angel \& Angel, 2006; Hansen-Kyle, 2005; Peel, McClure, \& Bartlett, 2005); productive aging (Butler \& Gleason, 1985); quality aging (Allardt, 1993; Haas, 1999; Lawton, 1991; Register \& Herman, 2006; Sarvimäki, \& Stenbock-Hult, 2000), and successful aging (Rowe \& Kahn, 1987). Among these, by far the most commonly identified construct in the literature is successful aging (Peel, Bartlett, \& McClure, 2004). The prevalence of successful aging is perhaps due to the use of the term in several large aging studies, e.g., the MacArthur Successful Aging Studies (Rowe \& Kahn, 1999; Seeman, Unger, McAvay, \& Mendes de Leon, 1999), the Harvard Study of Adult Development (Vaillant \& Mukamal, 1990), the Alameda County Study (Strawbridge, Cohen, Shema, \& Kaplan, 1996) and the Berlin Aging Studies (Baltes \& Carstensen, 1996; Freund \& Baltes, 1998). The notion of successful aging was also introduced to the lay public in the late 1990s (Bearon, 1996; Brody, 1996), with publication of the book, Successful Aging (Rowe \& Kahn, 1998).

Successful aging has largely focused on population-based epidemiological studies aimed at understanding genetic and biochemical causes of aging and on behavioral determinants of health with the potential to decrease chronic disease and to prevent or delay the onset of cognitive and physical disability (Rowe \& Kahn, 1987, 1997; Vaillant \& Mukamal, 1990). Other successful aging research has focused on ways elders perceive and adapt to changes of aging through self-regulation and coping strategies (Aspinwall \& 
Taylor, 1997; Baltes \& Baltes, 1990; Kahana \& Kahana, 1996; Schwarzer \& Taubert, 2002).

However, the potential of successful aging theory to help meet the goals of improving quality of life among older adults (Healthy People 2010, 2000; U.N., 2000; WHO, 1999) and eliminating health disparities (NIH, 2008) is limited by a lack of consensus among disciplines on definitions of successful aging (Bearon, 1996; Phelan \& Larson, 2002; Strawbridge, et al., 1996). Additionally, older adults' own criteria for successful aging have not been fully considered, especially in the spiritual or existential domain (Sadler \& Biggs, 2006). Unidimensional, normative criteria limit the potential for successful aging for individuals with chronic disease, disabilities, or low socioeconomic status (Bowling \& Dieppe, 2005; Bowling \& Iliffe, 2006; Depp \& Jeste, 2006). Finally, there is scant nursing theory focusing specifically on older adults' unique developmental perspectives and abilities to cope with changes associated with aging. This lack of theory limits nursing's ability to understand older adults' personal goals and priorities and to effectively support multidimensional coping efforts that older adults use to achieve successful aging (Flood, 2005; Wadensten \& Carlsson, 2003).

\section{Biomedical model.}

Theoretical foundations for the majority of successful aging studies are currently divided between two major models of successful aging, the biomedical and the psychosocial model (Glass, 2003; von Faber, 2001). The biomedical model, which tends to focus on disease rather than health, sets criteria for successful aging as: absence of disease and disability, independent physical and cognitive function, and active engagement (Rowe \& Kahn, 1997). These criteria exclude older adults with any degree 
of chronic disease or disability (Bowling \& Iliffe, 2006; Strawbridge, Wallhagen, \& Cohen, 2002; von Faber, 2001); limit the potential for success among those disadvantaged by socioeconomic, gender, racial, or cultural factors (Holstein \& Minkler, 2003); and impose normative criteria that do not reflect the full range of older adults' attitudes and values (Phelan, Anderson, \& LaCroix, 2004; Strawbridge, et al., 2002; von Faber, 2001).

\section{Psychosocial model.}

The second model of successful aging, the psychosocial model, defines successful aging as maximizing the potential to attain positive or desired outcomes while minimizing or avoiding, negative or undesirable outcomes (Freund \& Baltes, 1998). The psychosocial model emphasizes life satisfaction, social functioning, and psychological resources such as personal growth and social support (Bowling \& Dieppe, 2005) and focuses on factors such as self-regulation, self-efficacy, coping, and motivation to adapt to old age. While this model more fully captures the heterogeneity of the aging experience among older adults, it does not fully consider health and physical functioning. It is also highly individualized and subjective, applying criteria to measure success based on social norms and researchers' subjective biases, rather than measuring outcomes valued by elders (Schulz \& Heckhausen, 1996).

\section{Lay model.}

An additional model has been suggested, which Bowling and Dieppe (2005) term a lay model of successful aging. The lay model adds the unique perspectives of older adults themselves, incorporating personal values and attitudes toward criteria that constitute success. A scant number of research studies examine successful aging from the 
perspective of older adults, identifying criteria for success that have not been detected in either biomedical or psychosocial studies (Baltes \& Carstensen, 1996; Bowling \& Dieppe, 2005; Bowling \& Iliffe, 2006; Phelan, et al., 2004; Strawbridge, et al., 1996). While health and independent functioning are universally valued criteria among older adults, older adults also reported successful aging even in the presence of disease or disability. Additional criteria identified by older adults may be grouped into two categories: a) personally valued goals and existential beliefs, and b) social or environmental factors that influence health. A lay model, it was suggested, is needed to move beyond criteria for successful aging set by researchers, to add an existential or spiritual dimension not identified in other models, and to define non-normative criteria that will extend the potential for successful aging to older adults with chronic disease, disability, low socioeconomic status, and racial or ethnic minorities (Bowling \& Iliffe, 2006).

\section{Nursing model.}

Flood (2005) argued that although existing nursing theory may be extended to the care of older adults, there is a lack of theory specifically targeting older adults' unique developmental perspectives and the challenges of adapting to the changes of aging. Wadensten \& Carlsson (2003) conducted a systematic review of the literature which revealed that among 17 nursing theories examined, none described human aging or offered practical guidance for nurses caring for older adults. Only five theorists dealt with life span development, extended to late life - Benner and Wrubel, King, Roy, Travelbee, and Watson - and none of these theories considered interventions aimed specifically at older adults (Wadensten \& Carlsson, 2003). As a result, Flood argued, a model is not 
available to guide nursing research, practice, and policy development for older adults. Given the focus of the nursing profession on multidimensional health for individuals of all racial, ethnic, cultural, or socioeconomic groups, it appears particularly appropriate for nursing to propose a mid-range theory that may provide a theoretical foundation for successful aging that extends current theory to include a holistic, comprehensive view of successful aging available to all older adults.

\section{Statement of the Problem}

Competing perspectives on successful aging were identified in the academic literature, differing on definitions of successful aging and criteria for success, on factors that foster the potential for success, and on outcomes measured to determine when successful aging is achieved (Glass, 2003; von Faber, 2001). Older adults' unique perspectives on life and on aging have not been adequately considered (Bowling \& Dieppe, 2005; Phelan \& Larson, 2002; Phelan, et al., 2004), while older adults with disadvantages such as chronic disease, disabilities, minority status, or low-income are excluded by the focus on behavioral determinants of health rather than social and environmental factors (Holstein \& Minkler, 2003; Knickman \& Snell, 2002 ). Nursing lacks theory targeting the unique challenges and opportunities of late life (Flood, 2005;

Wadensten \& Carlsson, 2003). The result is skewed aging research, inadequate clinical practice standards, and poorly targeted social and health policy and program development (Bowling \& Iliffe, 2006). A different view of successful aging - one that is multidimensional and accessible, regardless of chronic disease, functional ability, minority status or socioeconomic level - is needed to extend the full potential for positive and successful aging to all older adults. 
A new mid-range nursing theory, the Theory of Successful Aging (Flood, 2005), appears to address this need. Evolved from the framework of life span development theory and a foundational nursing model, Flood's theory of successful aging offers a new nursing model, addressing the existential or spiritual domain, while responding to concerns about socioeconomic, cultural, racial, and gender bias, defining success through older adults' personal goals and values. A model of the theory in Figure 1 illustrates the relationships among the concepts in Flood's theory.

Figure 1

Flood's (2005) Model of Successful Aging

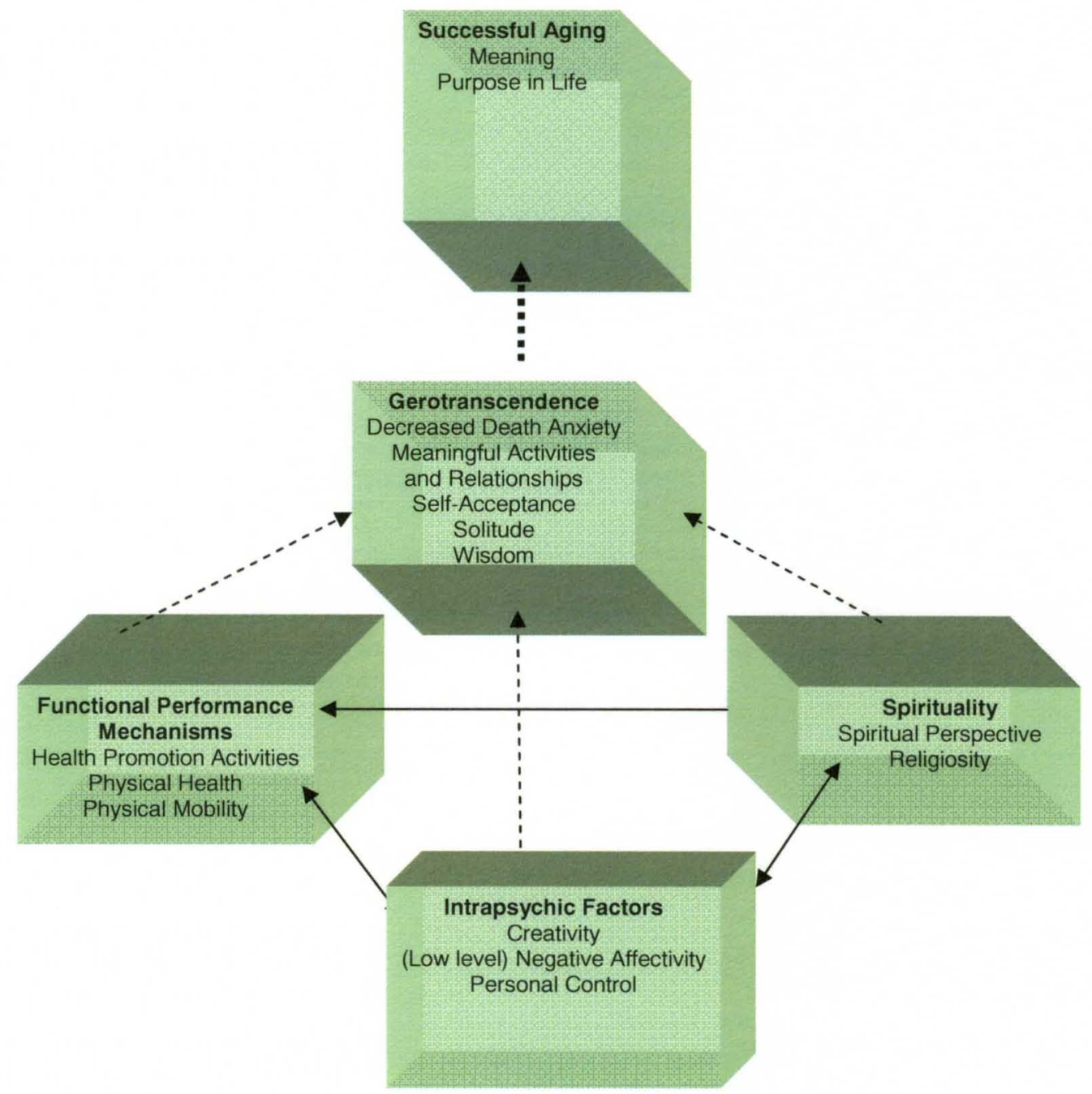


(Source: Flood, 2005)

Flood's (2005) theory proposes that two primary factors contribute to successful aging - adaptation and gerotranscendence. Adaptation, based on a foundational nursing theory (Roy, 1984; Roy \& Andrews, 1999), incorporates functional, intrapsychic, and spiritual coping. Only when adaptation is achieved through these three coping mechanisms is gerotranscendence a possibility, which in turn may lead to successful aging. Gerotranscendence, a late-life developmental stage, entails a shift in worldview from a materialistic and rationalistic perspective to a more mature and existential one (Flood, 2005; Tornstam, 1989, 2005). Together, according to Flood, adaptation and transcendence result in a holistic view of successful aging defined by the individual's perceived satisfaction in adapting to physical and functional changes of aging, while sensing spiritual connectedness and a feeling of meaning or purpose in life (Flood, 2003, 2005).

Rather than using externally-imposed criteria to judge success, Flood's (2003, 2005) definition is based on the individual older adult's assessment of his or her own success in achieving personally valued goals. These non-normative criteria extend the potential for success to older adults with chronic disease, disabilities, and to those disadvantaged by minority or low socioeconomic status. Inclusion of existential criteria identified by older adults, such as meaning in life and spirituality, shape a more multidimensional and comprehensive successful aging theory than those in either the biomedical or psychosocial models.

The Theory of Successful Aging (Flood, 2005) may offer direction to nursing and serve as a guide to research, practice, and policy to bring successful aging within reach of all older adults, but it is a new theory that has not yet been used in empirical studies. 
Purpose of the Study

The purpose of this research study is to explore Flood's (2005) Theory of Successful Aging, examining the relative contributions of adaptation and transcendence to successful aging among a sample of non-demented, low-income, racially diverse older adults, aged 65 and older, living independently or receiving assisted living services in a 390-unit non-profit continuing care retirement community (CCRC) operated by a charitable organization in an urban county of a Southern state, to examine the potential usefulness of the theory as a multidimensional guide to nursing research, clinical practice, and policy development.

To explore the propositions of Flood's (2005) theory, four research questions were explicated. Specific hypotheses were not stated, due to the early stage of the investigation and limited prior knowledge on which to base hypotheses.

\section{Research Questions}

1. What are the differences in successful aging, if any, between groups based on age, gender, race, marital status, education, income, function, perceived health, or number of chronic diseases?

2. What are the relationships among adaptation, transcendence and successful aging? Are both adaptation and transcendence predictors of successful aging?

3. To what degree do adaptation and transcendence explain any observed variance in successful aging among participants in the target population?

4. What are the differences in adaptation, transcendence, and successful aging between participants who report they are aging successfully and those who do not? 


\section{Conceptual Definitions}

Independent variables used in the study are adaptation and transcendence, with successful aging as the single outcome variable. Control variables are age, income level, functional status, perceived health, and number of chronic diseases. Conceptual and operational definitions for all variables are displayed in Table 1. For the purposes of this study, Flood's multidimensional $(2003,2005)$ definition of successful aging is used, that is, an individual's perceived favorable outcome in adapting to the accumulated physical and functional changes of aging, while experiencing a spiritual or existential sense of connectedness and meaning or purpose in life (Flood, 2005).

Adaptation, in Flood's (2005) theory, is a broad construct that reflects multiple strategies individuals use to cope and adjust to constraints or opportunities associated with aging, within functional, intrapsychic, and spiritual domains (Roy, 1984). Proactive coping (Schwarzer \& Taubert, 2002), is used for the study as an indicator of adaptation. Proactive coping integrates self-regulatory behaviors such as resource accumulation and realistic goal setting, with quality of life management strategies such as positivelyfocused striving for goals and personal growth, with a sense of well-being resulting from the ability to meet future challenges and opportunities (Greenglass, 2002). The propositions of proactive coping theory are congruent with Roy's theory.

Flood (2005) used Tornstam's $(1989,2005)$ definition of gerotranscendence, i.e., a major shift in an individual's perspective or worldview, involving a reassessment of one's place in the world and in relation to others, as the theoretical basis for transcendence. For the purposes of this study gerotranscendence is defined as selftranscendence (Reed, 2003), an inherent late life shift in perspective, with self-boundaries 
Table 1

Conceptual and Operational Definitions of Study Variables

\begin{tabular}{|c|c|c|}
\hline Variable & $\begin{array}{l}\text { Conceptual } \\
\text { definitions }\end{array}$ & $\begin{array}{c}\text { Operationalized } \\
\text { definitions }\end{array}$ \\
\hline $\begin{array}{l}\text { Predictor } \\
\text { variables: } \\
\text { Transcendence }\end{array}$ & $\begin{array}{l}\text { Self-transcendence is the expansion of } \\
\text { self-boundaries in multi-dimensional } \\
\text { ways: } \\
\text { (a) Intrapersonally - inwardly in } \\
\text { introspective activities } \\
\text { (b) Interpersonally - outwardly through } \\
\text { concerns about others' welfare } \\
\text { (c) Temporally - whereby the } \\
\text { perceptions of one's past and future } \\
\text { enhance the present, and } \\
\text { (d) Transpersonally -through } \\
\text { connections with a higher or greater } \\
\text { dimension (Reed, 2003) }\end{array}$ & $\begin{array}{l}\text { Self-transcendence Scale } \\
\text { (STS): } \\
\text { 15-item unidimensional } \\
\text { self-report questionnaire } \\
\text { with summative scoring on } \\
\text { a 4-point Likert-type scale. } \\
\text { May be administered as an } \\
\text { interview or questionnaire } \\
\text { (Reed, 1989b). }\end{array}$ \\
\hline $\begin{array}{l}\text { Adaptation, } \\
\text { Operationalized } \\
\text { as Proactive } \\
\text { Coping. }\end{array}$ & $\begin{array}{l}\text { Proactive coping integrates } \\
\text { (a) Self-regulatory behaviors such as } \\
\text { resource accumulation and realistic } \\
\text { goal setting, } \\
\text { (b) Quality of life management } \\
\text { strategies such as positively-focused } \\
\text { striving for goals and personal growth, } \\
\text { (c) Well-being and a sense of meaning } \\
\text { resulting from the ability to meet future } \\
\text { challenges and opportunities } \\
\text { (Schwarzer and Taubert, 2002). }\end{array}$ & $\begin{array}{l}\text { Proactive coping subscale } \\
\text { of the Proactive Coping } \\
\text { Inventory (PCI): } \\
\text { 14-item unidimensional } \\
\text { self-report questionnaire } \\
\text { with summative scoring on } \\
\text { a 4-point Likert-type scale. } \\
\text { May be administered as an } \\
\text { interview or questionnaire } \\
\text { (Greenglass, 2002) }\end{array}$ \\
\hline $\begin{array}{l}\text { Outcome } \\
\text { variable: } \\
\text { Successful } \\
\text { Aging }\end{array}$ & $\begin{array}{l}\text { Successful aging is "An individual's } \\
\text { perception of a favorable outcome in } \\
\text { adapting to the cumulative physiologic } \\
\text { and functional alterations associated } \\
\text { with the passage of time, while } \\
\text { experiencing spiritual connectedness, } \\
\text { and a sense of meaning and purpose in } \\
\text { life" (Flood, 2005) }\end{array}$ & $\begin{array}{l}\text { Successful Aging } \\
\text { Inventory (SAI): } \\
\text { 20-item scale with } 2 \\
\text { dimensions; summative } \\
\text { scoring on a 5-point Likert } \\
\text { (Flood, 2008). }\end{array}$ \\
\hline
\end{tabular}


Table 1, continued

\begin{tabular}{|c|c|c|}
\hline \multicolumn{3}{|l|}{$\begin{array}{l}\text { Other Variables: } \\
\text { Self-reported: }\end{array}$} \\
\hline Gender & Male / Female & \\
\hline Age & In Years & \\
\hline Race & Caucasian / African American / Other & $\begin{array}{l}\text { Investigator- } \\
\text { developed }\end{array}$ \\
\hline Marital status & $\begin{array}{l}\text { Married/ Never married/ } \\
\text { Divorced/separated/Widowed }\end{array}$ & instrument \\
\hline Educational level & $<$ HS / HS / > HS / College grad or $>$ & \\
\hline Perceived income & $\begin{array}{l}\text { Barely enough to get by/ enough to } \\
\text { get by/ enough to live in some luxury }\end{array}$ & \\
\hline Perceived health & Very good / Good / Fair / Poor & \\
\hline $\begin{array}{l}\text { Number of chronic } \\
\text { conditions }\end{array}$ & $\begin{array}{l}\text { Self-report of one or more of the } \\
\text { following: hypertension or high } \\
\text { blood pressure, arthritis, heart } \\
\text { disease, respiratory disease such as } \\
\text { chronic bronchitis, asthma, or } \\
\text { emphysema, cancer, diabetes, stroke } \\
\text { (CDC, 2008), and Other (CDC, n.d.). }\end{array}$ & \\
\hline Health Index & $\begin{array}{l}\text { Computed variables summing } \\
\text { perceived health and chronic disease }\end{array}$ & \\
\hline $\begin{array}{l}\text { Response to question: Are } \\
\text { you aging successfully? }\end{array}$ & Yes/No & \\
\hline $\begin{array}{l}\text { CCRC-Reported: } \\
\text { Income - rent levels }\end{array}$ & $\begin{array}{l}\text { Level 1) Section } 8,<\$ 18,000 \\
\text { Level 2) Section 236, } \$ 18 \text { to } 23,000 \\
\text { Level 3) Fair Market, } \$ 23 \text { to } 33,000^{*} \\
\text { (HUD, 2008a,b) }\end{array}$ & Sampling frame \\
\hline Functional status & Independent living / Assisted living & \\
\hline
\end{tabular}

* Below HUD (2008b) low-income level for County and State \& 300\% of federal poverty level. 
that are expanded (a) intrapersonally (introspection focused on one's philosophy, values, and dreams), (b) interpersonally (consideration of one's relationships to others and with the environment), (c) temporally (merging the past and future in a way that gives meaning to the present), and (d) transpersonally (a sense of existential connection with a greater power or the entire universe) (Reed, 1991a). Tornstam's and Reed's views of transcendence are largely congruent; both are based in life span development theory and involve a shift in world view in late life.

Control variables used in the study are age, income level, functional status, perceived health, and number of chronic diseases. Age is defined in years and categorically based on Garfein \& Herzog's (1995) classification of older adults as youngold (age 60-69), old-old (age 70-79, and oldest-old (age 80 and older). As self-reported income was found to be unreliable in a recent feasibility study (McCarthy, 2009) and participants receiving income-based services may be reluctant to report income, CCRC rent categories based on income, defined by HUD, are used as indicators of income. Perceived income is also measured, as one of three categories defined by Flood (2006, 2008). Health is measured as self-reported perceived health, defined as the participant's subjective assessment of his or her own overall physical and mental health, and as number of common chronic diseases conditions (Centers for Disease Control and Prevention, 2008). A composite index encompassing both perceived health and chronic disease is also computed.

Functional status is defined for the study as receipt of either independent living or assisted living services, based on functional criteria for each category established by CCRC policies and procedures. Independent living is defined by the CCRC as residence 
in an apartment (studio or one-bedroom) with a door that may be locked, private bath and kitchen, and services limited to assistance with instrumental activities of daily living (IADLs) such as shopping, meals, housekeeping, and laundry. Assisted living, as defined by the CCRC, includes all independent living services, with the addition of personal care, including one or more of the following: (a) more than incidental assistance with activities of daily living (ADLs) such as bathing and hygiene, dressing, transfer among bed, chair or wheelchair, ambulation, feeding, or toileting; (b) supervision of self-administration of medications; and/or (c) reminder or escort service to meals, activities, or other locations within the retirement community.

Other study variables include sociodemographic characteristics of self-reported gender, age, race, marital status, and education level, and perceived income, function, and health. The final study variable was a dichotomous response to the question, "Do you consider yourself to be successfully aging?"

\section{Delimitations and Assumptions}

Participation in the study is limited to non-demented older adults, aged 65 and above, living independently or receiving assisted living services at an urban, low-income, 390-unit continuing care retirement community. The CCRC was selected because it is the only identified retirement facility in the state that offers both independent housing and assisted living services for low-income older adults.

Assumptions of the study include a stratified random sample selected from a sampling frame consisting of all residents of the CCRC, aged 65 or older, living independently or receiving assisted services, unless the resident prefers not to be included in the sampling frame. Thus it is anticipated the sample will be somewhat representative 
of the target population, but the potential for self-selection bias exists, related to residents who opt out of the sampling frame or decline to participate when contacted.

It is also assumed participants in the study will respond to items on the survey questionnaires openly and honestly and that individuals with moderate to severe dementia would be unable to complete the survey process. The study assumes an accurate representation by the $\mathrm{CCRC}$ of policies and eligibility requirements, a correct report of residents' status regarding independent or assisted living services, and a complete list of names, telephone numbers, apartment numbers, and rent categories to establish the sampling frame.

Summary

With rapidly increasing lifespans and the impending arrival of the baby boom birth cohort, demands of the growing numbers of frail older adults will significantly increase the burden on economic, social, and health care systems. Adults in very late life, particularly low-income or racial minorities, are more likely to experience chronic disease and disability requiring maximal assistance at a point in their lives when financial and social resources may be diminished. Current aging theory excludes older adults with any degree of chronic disease or disability. Social conditions such as ageism and health disparities based on income and race limit the potential for successful aging, as currently defined, to older adults with sufficient economic, educational, and social resources to implement behavioral changes to improve health in late life, while social and environmental determinants that might improve health among low-income and racial minorities are not adequately explored. The lack of research on older adults' own view of 
successful aging misses important criteria for successful aging, such as a sense of purpose in life and a spiritual or existential perspective that add richness and meaning to life.

Comprehensive multidimensional theory on successful aging is needed to guide nursing research, practice, and policy. The Theory of Successful Aging (Flood, 2005) may meet this need. The theory merges biomedical and psychosocial models of successful aging. Flood's non-normative criteria for success allows for behavioral and environmental factors affecting health, and for existential or spiritual factors such as a sense of meaning or purpose in life. It is important to determine the usefulness of Flood's (2005) theory and its potential to provide a holistic, inclusive view of successful aging. The theory may provide a road map to guide planning and implementation of efficacious and cost-effective ways to cope with increased demands associated with the rapidly aging population. New aging theory is necessary to guide nursing research, practice, and policy, and to enable nursing to contribute to national and international goals such as increasing not only years of life, but also the quality of those years, and to eliminating health disparities. Achievement of these goals is important, not only for older adults and their caregivers, but for society as a whole.

Flood's successful aging theory proposed that adaptation and transcendence contributed to successful aging, as defined by the individual older adult. The review of the literature in the next chapter focuses on each of these major constructs (Flood, 2005). The theoretical background of each construct - first adaptation, then transcendence, and finally successful aging - was explored by considering related theories, to clarify the development of the construct of successful aging and the contexts in which it might 
occur. Subsequently, literature was examined to identify operationalized definitions of adaptation, transcendence, and successful aging for the study. 


\section{CHAPTER II}

\section{REVIEW OF THE LITERATURE}

From earliest times, old age has been valued and seen as rich with accumulated experience, learning, and wisdom. Writings by the ancient philosopher, Plato (360B.C.a,b, 399 B.C.), presaged questions about old age that are still debated today (McKee \& Barber, 2001). More recent aging theory has viewed aging alternately as: (a) an inevitable withdrawal or disengagement from active life, mutually beneficial for both the older adult and society as a whole (Cumming and Henry, 1961); (b) as persistence of mid-life activities and relationships throughout late-life, substituting new activities, but maintaining the same patterns and levels of activity from middle-age to old age (Havighurst, 1957, 1963); or (c) as an individual predisposition to view life and adapt to change in a consistent way throughout life, i.e., people who are active, socially-engaged, and adapt readily to change in mid-life retain those behaviors into old age; individuals who prefer a more contemplative, introspective lifestyle continue to do so; and those who approach life passively, with a negative outlook when young remain passive and negative in old age (Atchley, 1989).

To explore the theory of successful aging, and the relationships among its constructs, it is important to consider the theoretical development of adaptation, 
transcendence, and successful aging in literature from a number of disciplines and perspectives. To fully understand the meaning of each construct, related theories are examined, including major concepts and propositions, operationalized definitions, and the contexts in which theories have been applied among older adults. From this background, theoretical bases have been selected to support operationalized definitions for adaptation, transcendence, and successful aging for the study. Selection of theoretical foundations for each construct was based on congruence with Flood's theoretical definitions, evidence of theory testing, empirical studies among older adults, and the availability of appropriate, validated measurement instruments.

\section{Adaptation}

The first major construct in Flood's (2005) Theory of Successful Aging to be examined was adaptation. Theoretical development of the construct involved three adaptation theories - a foundational nursing adaptation model (Roy, 1984; Roy \& Andrews, 1999), a model of adaptation based on goal selection, optimization, and compensation (Baltes \& Baltes, 1990), and a theory of proactive adaptation (Kahana \& Kahana, 1996; Roy, 1984) - and two distinct perspectives on proactive coping (Aspinwall \& Taylor, 1997; Schwarzer \& Taubert, 2002). Schwarzer and Taubert's proactive coping subscale of the Proactive Coping Inventory (Greenglass, Schwarzer, Jakubiec, Fiksenbaum, \& Taubert, 1999) was selected to operationalize the construct of adaptation for the study.

\section{Theoretical Foundation}

Adaptation originated as a term used to describe an organism's ability to change 
physiologically to better suit its environment (Darwin, 1859). The concept of adaptation was later applied to a human's ability to find ways to adjust to a specific physical and social environment. Adaptation was an evolutionary or developmental process and a positive outcome of coping strategies used to adjust to or alter the environment in response to stress, enhancing the ability to survive or thrive. The outcome of adaptation was, by definition, always positive; adaptation only occurred if effective adjustments to or alterations in the environment were achieved (Smit, Burton, Klein, \& Street, 1999). Adaptive behaviors allowed an individual to balance internal needs and external demands; to monitor and evaluate goals, to observe and learn from observation and experience, and to adopt behaviors to change either the environment or personal attitudes and behaviors (Poon, Gueldner, \& Sprouse, 2003).

Adaptation was defined specifically for older adults as physical or behavioral modification used by an older adult in response to changes, stressors, or risks in the environment (Rimkus \& Melinchock, 2005, p. 37). Adaptation was a positive outcome of self-regulatory and coping behaviors that allowed older adults to cope and adjust effectively to the unique constraints and opportunities associated with aging (Schwarzer \& Taubert, 2002).

Coping was the mechanism through which adaptation occurred. Coping affected change in internal and/or external environments, allowing older adults to adapt to the inevitable losses, challenges and opportunities associated with aging (Baltes \& Baltes, 1990). Cognitive factors that shaped an individual's perception of stress and coping behaviors included self-regulation, self-efficacy, motivation, control, and appraisal of demands and resources, as well as temporal factors and the effects of uncertainty 
(Schwarzer \& Taubert, 2002). Proactive coping referred to coping not only in response to current stress, but coping behaviors aimed at avoiding or minimizing future stress or achieving future goals.

\section{Roy's adaptation model.}

The theory used as a conceptual basis for Flood's (2005) theory of successful aging was the Roy Adaptation Model (RAM) (Rice, 2000). Classified as a grand nursing theory or conceptual model (Fawcett, 2002; Polit \& Beck, 2003), the RAM had been used extensively to provide conceptual support for nursing intervention studies, educational curricula, and as a framework from which research hypotheses were generated and tested (Frederickson, Jackson, Strauman, \& Strauman, 1991). Roy drew on theory from physiology, psychology, sociology, and nursing, to develop the RAM, (Marriner-Tomey \& Alligood, 2006).

The RAM proposed that the person, a biopsychosocial being, was in constant interaction with the environment, continually adapting to environmental stimuli. The goal of adaptation was to attain and maintain integrity, or wholeness, through use of two coping mechanisms which might be innate or acquired: regulator and cognator. The multidimensional theory encompassed both physiological responses to stimuli (the regulator mechanism) and psychological adaptations which influenced interpretation of stimuli (cognator mechanism). Adaptation occurred within four modes or domains: physiologic/physical, role function, interdependence, and self-concept/group identity. Adaptive behaviors were termed integrated, compensatory, and compromised, reflecting three levels of effectiveness. Integrated adaptation was effective in allowing the individual to maintain integrity within the environment on a day-to-day basis, while 
compensatory adaptation reflected threats or challenges in the environment requiring active use of adaptation to maintain integrity. Finally, compromised adaptation indicated the inability of the person to adapt sufficiently to maintain health, necessitating nursing intervention to restore the adaptive balance between person and environment to reachieve integrity.

The RAM served as a conceptual framework for multiple studies and generated numerous testable hypotheses, but operationalization of the RAM was less common. A systematic review examining instruments designed to measure concepts of the RAM identified 123 instruments used in 231 studies over 30 years. Among 20 instruments which met study criteria, only 14 were judged as highly useful, three were moderately useful and two not recommended for use with the RAM (Barone, Roy, \& Frederickson, 2008). No measure of the RAM as a measure of adaptation among older adults for the dissertation study was identified. A limitation of the RAM was that the model did not take a life span development perspective, focusing on the human's potential for change over time (Wadensten \& Carlsson, 2003). Arguably, the RAM was not congruent with current humanistic nursing values (Reed, Shearer, \& Nicoll, 2004); the nurse-role was viewed as the arbiter of decisions on an individual's needs, while the patient was seen as the recipient of nursing intervention, rather than a partner in pursuit of health.

\section{Selection, optimization, and compensation metamodel.}

Another adaptation theory identified in the literature which was congruent with the study's conceptual definition of adaptation was Baltes and Baltes' (1990) Selection, Optimization, and Compensation (SOC) Model of Successful Aging. Baltes and Baltes (1993) argued human development continued throughout the life span, with 
psychological and behavioral processes used to maintain and achieve goals resulting in successful aging. The SOC model defined success as goal attainment and successful aging as minimization of losses and maximization of gains (Baltes \& Carstensen, 1996). Adaptation was defined as the process through which older adults coped with the inevitable challenges and losses of aging - as well as gains arising from wisdom and experience - to achieve successful aging (Freund \& Baltes, 1998).

The multidimensional SOC metamodel proposed three processes of selfregulation which individuals used throughout life to adapt to, and effect change in, their environment: selection, optimization, and compensation. Selection referred to adaptations made when energy and stamina decreased with age, thus older adults became more selective about activities or people worth the investment of limited resources such as time and energy. Optimization involved tenaciously maintaining established goals, finding new ways to accomplish goals in the face of change. Finally, compensation reflected the necessity for choosing new activities, making new social connections, and substituting alternative methods when old methods were no longer possible or effective (Baltes \& Baltes, 1990).

The SOC model was developed in conjunction with the Berlin Aging Study (BASE), a multidisciplinary, longitudinal study which followed a core sample of older adults, aged 70 to over 100 years, $(\mathrm{N}=516)$ from $1990-1993$, with surviving participants examined six times to the present (Freund \& Baltes, 1998). The theory was relatively concrete, concepts were well-defined, and the model was parsimonious, with only three major concepts, and had been extensively tested in a series of studies which confirmed all 
major propositions (Abraham \& Hansson, 1995; Baltes, M., Wahl, \& Reichert, 1991;

Duke, Leventhal, Brownlee, \& Brownlee, 2002; Lang, Rieckmann, \& Baltes, M., 2002).

A well-validated German-language measurement instrument was developed for the SOC metamodel specifically for older adults and used with good reliability (Baltes, P., Baltes, M., Freund, \& Lang, 1999) in multiple empirical studies in Europe among middle-aged and older adults (Freund \& Baltes, 1998, 2000, 2002a, 2002b). In the United States, an English-language, computer-based version of the SOC scale was used in studies among children, adolescents and working age adults (B. Baltes \&Heydens-Gahir, 2003; Gestdottir \& Lerner, 2007; Young, Baltes \& Pratt, 2007; Zimmerman, Phelps, \& Lerner, 2007), but not among older adults. A recent feasibility study (McCarthy, 2009) considered the use of the SOC for the study but found that older adults were confused by the instrument's formatting, which included both dichotomous and Likert-type responses to the same set of items. The instrument was not significantly correlated with successful aging and regression models using the SOC scale, with measures of transcendence, did not predict successful aging.

\section{Proactive adaptation.}

A second theory identified in the adaptation literature, which was also related to successful aging, was Kahana and Kahana's (1996) Proactive Adaptation Model. The proactive adaptation model was one of few adaptation models outside nursing to focus on physical and mental health, as well as psychological adaptation mechanisms. The model was developed specifically for older adults, by an interdisciplinary team of social gerontologists, including sociologists, psychologists, nurses, and other social science disciplines (Kahana, E., n.d.). The proactive adaptation model argued that older adults 
could exercise control and effect positive outcomes even in the face of increasing physical frailty and social losses associated with aging (Kahana \& Kahana, 1996).

Chronic illness and functional disability in late life were viewed as normative stressors of aging.

Adaptation was not viewed as a passive response to current risks and losses but as a future-oriented, dynamic, ongoing process of goal-setting and proactive efforts to attain those goals to achieve successful aging, making sense of life and maintaining identity (Kahana, Kahana, \& Kercher, 2003). Kahana and Kahana (1996) proposed that older adults not only adjusted in response to an actual imbalance between needs and resources (corrective adaptations) but also exercised strategies to build resources for anticipated future needs (preventive adaptations). Specific corrective adaptations included strategies to access formal and informal social support; substitute alternative social roles when losses such as widowhood or illness occurred; and modifying activities or environmental conditions to enhance comfort and safety (Kahana, et al., 2002). Preventive adaptations included health promotion behaviors; planning ahead for potential financial and care needs; and helping others, thereby building future social support. A later revision of the theory proposed a third classification, emergent adaptations, defined as technology use, health care consumerism, and striving for self-improvement (Kahana, Kahana, \& Kercher, 2003). Proactive coping efforts were facilitated by both internal and external resources. Internal resources included hopefulness, self-esteem, and altruism, while external resources, which might be generated by internal resources or by proactive adaptations, were defined as financial resources and available social supports (Kahana, Kahana, \& Kelley-Moore, 2008). 
Theory development (Kahana \& Kahana, 1996) was conducted over 10 years in a longitudinal study among healthy old-old adults living in a Florida retirement community $(\mathrm{N}=1000)$, and was extended to urban and minority elderly, frail, institutionalized oldold adults, and young-old adults relocating to retirement communities (Kahana, n.d.). Research centered on environmental and social influences on well-being of older adults, responded to social trends (Kahana, Kahana, \& Kercher, 2003), and was validated in a number of empirical studies (Kahana, Chirayath, Wisniewski, \& Kercher, 2003; Kahana, Kahana, and Kelley-Moore, 2005; Kelley-Moore, Schumacher, Kahana, \& Kahana, 2006).

Proactive adaptation was operationalized using a battery of instruments to measure health, function, and psychological processes (E. Kahana, electronic communication, June 7, 2008). Operationalized concepts included physical stressors (functional measures for ADL's, IADL, number of chronic illnesses, and dichotomous response to a question on serious illness experienced in the past year), social stressors (count of the number of recent social losses experienced by the respondent), internal resources (eight items that asked about active coping strategies, three about religious coping, and five items focused on life satisfaction), external resources, both financial (income) and social (number of living children, widowed), and proactive adaptations, operationalized using three questions asked about the past year (how easy it was to ask for support; if future plans had been made, such as a trip, purchase, new project; and if plans for future disability had been discussed) and a fourth item on the number of hours of physical activity in the past week (Kahana \& Kahana, 1996). 


\section{Concept of proactive coping.}

Proactive coping, a relatively new conceptual perspective on coping, was futureoriented, focusing on appraisal of goals to be met or stresses to be averted (Sohl, 2008a,b). As with proactive adaptation, proactive coping occurred before a stressor was present, rather than only in reaction to existing stress (Greenglass, 2002). The concept of proactive coping focused on a set of coping strategies used to achieve adaptation, as did Roy's adaptation model (1984), and shared a focus on goal achievement with the model of selection, optimization, and compensation (Baltes \& Baltes, 1990). Two distinct conceptualizations of proactive coping (Aspinwall \& Taylor, 1997; Greenglass, 2002;

Schwarzer \& Taubert, 2002) were identified in the literature, encompassing slightly different approaches. Both focused on older adults' active management of internal and external environments to achieve positive outcomes, by integrating processes of personal quality-of-life management with self-regulatory goal attainment strategies. Building and conserving resources was a primary focus of both views of proactive coping.

Aspinwall and Taylor proactive coping theory.

Aspinwall and Taylor $(1997,2005)$ described proactive coping and self-regulation strategies used to avoid or delay the effects of future stresses associated with aging, focusing on identifying specific components of the coping process. Based on social cognitive theory (Bandura, 1977, 2004), Aspinwall (2005) described five steps in the process of proactive coping: a) first individuals worked to accumulate resources (social, financial, educational) and build coping skills over time; b) next, the environment was continually screened for danger; and c) potential stressors were recognized and appraised as either threat or opportunity; then d) initial coping responses were activated, including 
information gathering and cognitive activities such as planning ways to minimize or prevent future stress; and finally, e) after initial coping efforts, feedback was elicited which shaped persistence of coping efforts and selection of future coping strategies.

Aspinwall and Taylor $(1997,2005)$ proposed that proactive coping strategies varied, according to the goals selected, the nature of potential stressors, and the perceived threat of a specific stressor. The certainty of a threatening event and how far in the future the threat might occur influenced appraisal of a threat, choice of initial coping strategies, and degree of persistence in coping. While all individuals proceeded along the same steps in the process of proactive coping, cognition and behavioral effort also varied within and between individuals, across time, and across types of stressors (Ouwehand, de Ridder, \& Bensing, 2007). An advantage of Aspinwall and Taylor's proactive coping theory was its ability to account for differences in skills and resources among individuals at each stage of the process (Aspinwall \& Taylor, 1997), thus accommodating cultural, racial, and socioeconomic differences.

It should be noted that the perspective on aging taken in Aspinwall \& Taylor's (1997) proactive coping theory was essentially negative. The theory dealt only with proactive coping aimed at preventing or minimizing the effects of losses commonly experienced in late life, such as loss of health, self-image, autonomy, or valued relationships. The theory did not focus on proactive coping used to achieve goals or promote positive aging experiences.

Schwarzer and Taubert proactive coping theory.

Schwarzer and Taubert (2002) shared Aspinwall and Taylor's (1997) focus on future orientation, incorporating temporal and certainty factors, but argued that the 
selection of coping behaviors varied depended on the nature of stressors. Schwarzer and Taubert's proactive coping theory distinguished between four types of coping behaviors related to the nature of stressors: reactive (past harm/loss), anticipatory (imminent threat), preventive (uncertain future threat), and proactive coping (upcoming, potentially positive challenges). Reactive coping, according to Schwarzer and Taubert, aimed at ongoing coping efforts to alleviate or compensate for a past experience of loss or harm, by adjusting goals, finding benefit, or searching for meaning in past experiences. Anticipatory coping involved short-term stressors that were perceived as threatening and were expected to occur with reasonable certainty in the near future; coping behaviors focused on risk-management of known risks. Resources might be expended to prevent or limit a threat or to re-define the meaning of the threat.

Preventive coping dealt with uncertain threats in the future. Preventive coping strategies aimed at minimizing the impact or consequences of a threat, or decreasing the likelihood of occurrence of a threat (Schwarzer \& Taylor, 2002). Preventive coping involved risk-management of unknown risks in the far distant future. Proactive coping, on the other hand, focused on goal achievement. Proactive coping concerned building up resources to maximize the potential for a positive outcome; it allowed for the possibility of achieving challenging goals in old age and continuing lifespan development into the future. Schwarzer and Taylor's view of proactive coping was unique; it differed from previously considered coping theories by viewing stress as a potential for positive gain rather than only an avoidance of a negative threat. Using proactive coping, future risks might be appraised as opportunities for personal growth, finding meaning, and a sense of purpose in life. Accumulating resources, developing skills, and long-term planning were 
key strategies for proactive coping, and both ambitious goal setting and tenacious goal pursuit were important self-regulatory tasks.

Greenglass, Schwarzer, Jakubiec, Fiksenbaum, \& Taubert (1999) described three antecedents of a proactive belief system. First, the fully proactive individual was intrinsically motivated to take responsibility to make things happen. Second, the proactive individual had a positive disposition toward life in general; the proverbial glass was always half full rather than half empty. Third, the proactive individual was oriented toward active coping rather than passive coping, actively building up resources, consciously conserving available resources, and skillfully mobilizing resources when needed. Proactive coping reflected a trend in psychology away from pathology and dysfunction toward a more positive, optimistic approach that emphasizes health and the promotion of well-being (Folkman, 1997). Proactive coping, according to Schwarzer and Taubert (2002), was a holistic concept that allowed for strategies aimed at coping with both losses and achieving potential gains in late life.

\section{Comparison of proactive coping theories.}

The two proactive coping theories under discussion (Aspinwall and Taylor, 1997; Schwarzer and Taubert, 2002) differed significantly on the potential outcomes of proactive coping (Sohl, 2008b). Aspinwall and Taylor's view of proactive coping appeared to be equivalent to Schwarzer and Taubert's definition of preventative coping, which perceived stressors as threats rather than potential opportunities. The purpose of proactive coping, in Aspinwall and Taylor's theory, was to avoid a potential negative outcome in the future. On the other hand, Schwarzer and Taubert's (2002) view of proactive coping focused on the potential for positive outcomes. 
A study among college students $(\mathrm{N}=300)$ compared the two conceptualizations, using exploratory and confirmatory factor analysis and structural equation modeling to examine the ability of each conceptualization of proactive coping (Aspinwall and Taylor, 1997; Schwarzer \& Taylor, 2002) to predict well-being and to identify specific coping and self-regulatory mechanisms used in proactive coping (Sohl, 2008). Two subscales of the Proactive Coping Inventory (PCI) (Greenglass, et al., 1999) were used to measure the two conceptualizations of proactive coping. Schwarzer and Taubert's conceptualization of proactive coping was measured using the 14-item proactive coping subscale of the PCI, whereas Aspinwall and Taylor's (1997) view of proactive coping was measured using the 10 -item preventive coping subscale of the PCI. The study found that proactive coping, measured by the proactive coping subscale of the PCI, was significantly positively associated with well-being, supporting Schwarzer and Taubert's (2002) positively focused definition of proactive coping as tenacious goal pursuit and striving for personal growth and suggesting the proactive coping subscale was most likely to measure adaptation related to the positive construct of successful aging. Aspinwall and Taylor's (1997) negatively focused definition of proactive coping through prevention of negative outcomes was not significantly related to well-being.

Additionally, the Proactive Competence Scale (PCS), a 21-item instrument, developed among older adults 50 and 75 years of age, designed to measure the five steps of Aspinwall and Taylor's (1997) proactive coping process (Bode, de Ridder, Kuijer, \& Bensing, 2007) was used to identify specific coping and self-regulatory strategies used in proactive coping. Aspinwall and Taylor's conceptualization of proactive coping and the Proactive Competence Scale (PCS) best explained specific self-regulation processes and 
quantified proactive coping competencies. Among specific coping and self-regulatory strategies measured on the PCS, resource accumulation and realistic goal setting were particularly successful in explaining how proactive coping affected well-being when facing an upcoming stressor (Sohl, electronic communication, June 6, 2008).

In summary, the comparison of the two conceptualizations of proactive coping (Aspinwall \& Taylor, 1997; Schwarzer and Taubert, 2002) found that Schwarzer and Taubert's view of proactive coping focused on the possibility of achieving positive outcomes, rather than avoiding negative outcomes (Aspinwall \& Taylor, 1997), and appeared to be most closely aligned with successful aging (Flood, 2005). The PCI was significantly correlated with well-being, tenacious goal pursuit, and striving for personal growth and meaning in life (Sohl, 2000), concepts closely related to Flood's theory. The proactive coping subscale of the PCI was congruent with the coping processes in Roy's adaptation model (1984) used as the theoretical basis for the construct in Flood's (2005) successful aging theory, measuring functional coping strategies of cognitive goal recognition, resource accumulation, and goal-seeking behavior, was positively associated with intrapsychic factors such as a positive outlook and a disposition toward both assimilative and accommodative coping, and with a focus on spiritual factors such as personal growth, well-being, and finding meaning in life. Finally, the PCI proactive coping subscale (Schwarzer and Taubert, 2002) best detected the overall effects of adaptive coping, while the PCS (Aspinwall \& Taylor, 1997), identified specific coping strategies (Sohl, 2008b). Given the purpose of the proposed study, which is to measure the presence of proactive coping rather than the nature of the concept, Schwarzer and 
Taubert's (2002) proactive coping subscale of the PCI was considered for a measure of adaptation for the study.

Operational Definition of Adaptation: Proactive Coping

The 55-item Proactive Coping Inventory (PCI) was composed of 7 subscales, designed to be used singly or together. The PCI was comparable to measures of proactive attitude (Schwarzer, 1999), perceived self-efficacy (Schwarzer, 1998), and to the Brief COPE scale (Carver, 1997). The seven subscales, designed to be used singly or in combination, included: proactive coping, reflective coping, strategic planning, preventive coping, instrumental support seeking, emotional support seeking, and avoidance (Greenglass, et al., 1999). The 14-item proactive coping subscale combined autonomous goal setting with self-regulatory goal attainment cognitions and behaviors. The 11-item reflective coping subscale included brainstorming, analyzing problems, evaluating resources, and comparing possible courses of action. The 4-item strategic planning involved breaking down tasks into manageable parts, then setting a schedule to accomplish a goal. The 10 -item preventive coping subscale was threat-based, dealing with anticipating future stressors and initiating advance planning to avoid or minimize a threat. Instrumental support seeking used 8 items to identify methods of gaining support from others, such as obtaining advice, information and feedback from people in one's social network. The 5-item emotional support seeking subscale dealt with short-term emotional distress by talking things over with others, attempting to gain sympathy, and seeking companionship. Finally, the 3-item avoidance subscale used delay or withdrawal from a situation to evade taking action (Greenglass, et al., 1999). 
The proactive coping subscale of the PCI (Greenglass, et al., 1999) selected to operationalize adaptation for the study was positively associated with planning, positive reframing and a degree of acceptance, and was negatively related to depression, selfblame, denial, and behavioral disengagement. The proactive coping subscale was the only one of the seven subscales that was consistently and negatively related to depression. Higher scores on the proactive coping subscale were positively associated with intrapsychic factors such as a positive outlook and a disposition toward both assimilative and accommodative coping (Greenglass, et al., 1999).

The PCI was used with a number of older adult samples (Greenglass, et al., 1999; Greenglass, Fiksenbaum, \& Eaton, 2006; Simons, 2002). In a study that examined the predictive effect of locus of control, proactive coping ability, and self-efficacy on life satisfaction of young, middle-aged, and older adults $(\mathrm{N}=240)$, the $\mathrm{PCI}$ found younger adults were most proactive in coping with stress, and older adults were least proactive, although older adults were most satisfied with life (Simons, 2002). A study among 224 community-dwelling older adults attending a community center (mean age 75) used the Proactive Coping subscale of the PCI to examine proactive coping, social support, daily hassles, functional disabilities, and living arrangements (Fiksenbaum, et al., 2006). Proactive coping was significantly negatively related to hassles and functional disabilities. Proactive coping and living arrangements were positively related to social support. Test/re-test stability of the proactive subscale of the PCI was also demonstrated $(.80-.85)$.

A recent feasibility study (McCarthy, 2009) found statistical significance for a model regressing proactive coping, operationalized using the proactive coping subscale, 
and self-transcendence on SAI $(F=4.592, p=.027)$, explaining $36.5 \%$ of the variance. While the bivariate correlation of PCI with SAI $(r=+.340)$ was not statistically significant, the correlation was markedly larger than other instruments for adaptation tested in the feasibility study (two versions of a SOC scale) and might be significant with a larger sample and greater power. Cronbach's alpha was .62 for the internal reliability of the proactive coping subscale in the feasibility study. The instrument was also brief and well-received by participants, supporting the selection of the Proactive Coping Subscale of the PCI to operationalize adaptation in the study.

Having considered the theoretical background for the construct of adaptation, examined empirical literature on its use among older adults, and operationalized adaptation for the study as the proactive coping subscale of the PCI (Greenglass, et al., 1999), the next section of the dissertation considered the second predictor of successful aging in Flood's (2005) theory, that is, transcendence. As with adaptation, the theoretical background of the construct in life span development theory was briefly explored, followed by examination of two theories of transcendence, Tornstam's $(1989,2005)$ theory of gerotranscendence and Reed's (1991a, 2003) theory of self-transcendence. Literature supporting an operational definition of transcendence for the study, its testing and use in empirical studies among older adults was then considered.

\section{Transcendence}

\section{Life Span Development Theory}

Life span development theory described stages in a process of growth and development, beginning in infancy and progressing throughout life. Individuals' progress through stages of development varied; some progressed farther than others, and not all 
achieved full potential. Erikson's $(1963,1980)$ stages of personality development proposed wisdom was the final stage in life span development, the positive outcome of an inherent conflict between ego-integrity and despair. A later revision of Erikson's theory proposed an additional stage of development (Erikson \& Erikson, 1997), moving beyond wisdom to transcendence, marked by a broader perspective of self, a sense of cosmic union with the universe, where time existed only as now, or maybe the near future, space was reduced to the radius of an one's mobility, and death was the way of all living things. Maslow's $(1943,1968)$ hierarchy of needs described self- actualization, that is, self-fulfillment or the ability to reach one's full potential, as the highest level of development in late life. Like Erikson, Maslow's (1971) later revised the hierarchy to suggest transcendence was a natural extension of self-actualization. Maslow stated older adults experienced transcendence as an inner spiritual dimension, co-existing with the everyday world. Maslow (1969) noted the meaning of transcendence was founded in the verb transcend, that is, to rise above or move beyond time, culture, self, and others. Transcendent behaviors included: moving beyond one's own weaknesses, fears, and dependency; to transcend the opinions or expectations of others, such as social conventions, family roles, or a focus on others to the exclusion of self; and to achieve a sense of unity with one' self, the universe, others - especially the next generation - and a higher power as perceived by the individual.

Having explored the nature and development of the construct of transcendence in life span development theory, the focus of the review of the literature moved to two theories of transcendence in late life. The first theory considered was a Theory of Gerotranscendence (Tornstam, 1989, 2005), which provided the theoretical basis for the 
construct of transcendence in Flood's Theory of Successful Aging (2005). The second transcendence theory, the Theory of Self-transcendence (Reed, 1991a, 2003), was a midrange nursing theory which fused life span development theory and another foundational nursing theory (Rogers, 1970) with a specific focus on health in a multidimensional view of transcendence near the end of life.

\section{Theory of Gerotranscendence}

Tornstam $(1989,2005)$ proposed a theory of gerotranscendence to describe the potential for psychological development in late life. Tornstam defined gerotranscendence as a shift in one's perspective, from a materialistic and rational view of the world to a more existential and transcendent one. Tornstam (1997a) argued that gerotranscendence usually led to an increase in life satisfaction. Gerotranscendence was a final stage in a natural progression towards maturation and wisdom marked by a shift to an existential viewpoint; greater acceptance of self and others; increased selectivity of relationships and activities based on personal choice rather than the expectations of others; a greater need for solitude; decreased fear of death; and accumulated wisdom gained from life experience (Tornstam, 2005). An amalgamation of disengagement theory and life span development theory, the theory of gerotranscendence (Tornstam, 1989, 2005) differed from other theories of aging. Rather than viewing transcendence as a trait gradually developed throughout the life span, gerotranscendence was envisioned as a sudden, distinct shift in reality in late life to a more cosmic or existential state (Wadensten $\&$ Carlsson, 2005). Gerotranscendence theory proposed older adults' view of reality was qualitatively different from mid-life. 
Gerotranscendence was a multidimensional construct with three dimensions (Tornstam, 1997a): the self, the social and the cosmic. The self or individual level was marked by decreased interest in material matters, less self-centeredness, and a need for contemplation. The social level involved a desire for solitude and decreased concern for superficial social contacts or expectations, while the cosmic dimension involved a blurring of past and future, an affinity for the next generation, and a sense of connectedness with one's inner self, nature and the universe (Tornstam, 1989). The need for contemplation, solitude, and a focus on the inner self specified in gerotranscendence was congruent with disengagement theory, but gerotranscendence differed because the impulse to disengage, to dispense with external demands, and to turn inward, was countered by expanded concern for the next generation (generativity) and a strong desire to retain a sense of purpose in life (Tornstam, 2000, 2005). McKee and Barber (2001) noted that when the shift to gerotranscendence occurred, older adults experienced an interested detachment from everyday concerns, but the impulse to disengage was buffered by concern for the next generation and a need to continue contributing.

Tornstam's $(1989,2005)$ theory was tested in a relatively small number of empirical studies. An early iteration of gerotranscendence theory with only two dimensions, the self and the cosmic, was tested using a 10-item investigator-developed questionnaire in a mail survey in 1986 and repeated in 1990 among 912 noninstitutionalized Danish men and women, aged $74-100$. The longitudinal study supported the hypothesis that gerotranscendence was associated with social activity, coping and life satisfaction (Tornstam 1994), but the data did not support the hypothesis that gerotranscendence was associated with age. In 1991, Tornstam (2005) conducted a 
second study among 2,200 Swedish men and women, aged 20 to 85 , who completed the same mail surveys, using the gerotranscendence scale. Results were not consistent with the theory or the previous studies; perception of time, fear of death, and material needs did not vary in the direction anticipated.

A phenomenological qualitative study used semi-structured interviews among a sample of older adults, aged 52-97, who had attended a lecture on gerotranscendence one month previously, to examine self-attitudes and developmental changes in the participants' views of the world. Participants in the qualitative sample reported awareness of a more cosmic dimension to reality and a less narcissistic view of self, related to gerotranscendence. Findings were contrary to those expected, and prompted a revision of the theory, adding a social dimension to the original cosmic and self dimensions (Tornstam, 2005). Tornstam (1996) also tested the theory clinically (1996); the theory of gerotranscendence was introduced to a sample of 90 staff members in a Swedish nursing home, followed six months later by a mail survey. Results supported a correlation between staff interpretations of the theory and their perceived reality in caring for older adults.

Uses of the gerotranscendence theory by other researchers were identified. Wadensten and Carlsson (2005), in a qualitative study, introduced gerotranscendence to a sample of older women who reported that the idea of gerotranscendence introduced them to a more positive view of ageing that increased self-acceptance. A second study applied the theory of gerotranscendence to gerontological nursing practice, concluding that nursing staff often confused the natural tendency toward solitude that was part of the developmental stage of gerotranscendence with withdrawal, isolation, and depression. As 
a result, increased activity was often inappropriately and unnecessarily encouraged (Wadensten \& Carlsson, 2003).

A study among 928 older Dutch adults examined the relationship of the cosmic transcendence subscale of Tornstam's Gerotranscendence scale (Braam, Bramsen, van Tilburg, Ploeg, \& Deeg, 2006). Findings substantiated a positive relationship between cosmic transcendence and meaning in life among participants who were less involved in religion, who were women, who were aged 75 or older, or who were widowed. The study concluded that the personal relevance of cosmic transcendence depended on cultural factors, such as secularization and was of particular importance in understanding the life view of women, the older old, and the widowed. An English-language abstract reported a German study which examined Tornstam's gerotranscendence scale, using data from a sample of adults aged 56-76 years $(\mathrm{N}=556)$. Two subscales were identified, congruent with the concepts of cosmic transcendence and ego-transcendence proposed in Tornstam's theory $(1996,2005)$. Scores on both subscales were higher for the older old, unmarried; divorced or widowed participants, for those with physical limitations, or with depressive complaints. Scores on the cosmic subscale varied by type of religion, and the ego-transcendence subscale by education and number of social contacts. The strength of the associations was modest and only a small amount of the variance was explained (Braam, Deeg, van Tilburg, Beekman, \& van Tilburg, 1998). Also, study of 1,845 older parents participating in the 1995/1996 cycle of the Amsterdam Longitudinal Aging Study supported a positive association between emotional support exchanged with children, feelings of loneliness later in life, and higher levels of cosmic transcendence (Sadler, Braam, Broese van Groenou, Deeg, \& van der Geest, 2006). 
Jönson and Magnusson (2001) severely criticized Tornstam's (1989) gerotranscendence theory, comparing the theory to the New Age movement and romantic Orientalism, and stating that Tornstam was attempting to make aging an enchanted fantasy rather than recognizing it as a practical reality. Jönson and Magnusson stated that Tornstam's theory was also empirically weak. A critical review of empirical studies of gerotranscendence found that in Tornstam's 1994 study, while some support was found for social activity, coping and life satisfaction, contrary to expectations, transcendence scores were not correlated with age. In another study (Tornstam, 1997b), results again were inconsistent and contradictory on a number of important hypotheses.

In summary, gerotranscendence was a well-developed theory, closely related to both disengagement theory and life span development theory. Gerotranscendence defined three domains, the self, social and cosmic and proposed a shift in perspective in old age to a cosmic perspective, qualitatively different from mid-life. From a theoretical viewpoint, the specifications of the theory appeared to be congruent with aging theory and nursing practice. However, while conceptually attractive, empirical evidence for the theory was limited, with inconsistent results. While gerotranscendence theory provided a conceptual definition for transcendence in the theory of successful aging (Flood, 2005), a second theory on transcendence was identified, which was also congruent with Flood's conceptual definition of transcendence.

\section{Theory of Self-transcendence}

A second perspective on transcendence was identified in the mid-range nursing Theory of Self-transcendence (Reed, 1991a, 2003). Reed described self-transcendence as a developmental imperative, an innate human characteristic much like other 
developmental processes. Self-transcendence, according to Reed, was expressed within five contexts: a) creativity; b) spirituality; c) generativity; d) a sense of oneness with nature; and e) mystical experiences. Unlike the theory of gerotranscendence, selftranscendence was a gradual, nonlinear process of development throughout middle- and old-age, rather than an abrupt shift in perspective. Reed's (2003) mid-range nursing theory compared the theory of self-transcendence to continuity theory, but argued people were, "more contextual, more pragmatic, more spiritual, and more tolerant of ambiguity and the paradoxes inherent in living and dying" in maturity than in mid-life (p. 147-8).

The theory of self-transcendence proposed a deeper awareness of self and environment, with a broader perspective, near the end of life. Reed's (2003) selftranscendence theory was multidimensional, defining self-transcendence as an expansion of self-boundaries within four domains: intrapersonally (toward greater awareness of one's philosophy, values, and dreams), interpersonally (to relate to others and one's environment), temporally (to integrate one's past and future in a way that has meaning for the present), and transpersonally (to connect with dimensions beyond the typically discernable world).

The theory of self-transcendence (Reed, 1991a, 2003) was developed by merging life span development theory with Rogers' (1970) conceptual model. According to Reed (1991a), whereas life span theories saw development as unidirectional, moving from the egocentric and everyday toward the detached and existential, Rogers' model viewed human development as a continuing interaction between humans and their environments, constantly open to innovative change, moving in a nonlinear process toward integration. 
In Rogers' model, an individual's life experiences were processed and organized into conceptual or self-boundaries that made sense to individuals and shaped their worldview.

Reed (1991a) added elements of Rogers' (1970) conceptual system to existing life span development theory, resulting in a theory of self-transcendence that was less complex and more concrete than gerotranscendence, and particularly relevant to nursing. Reed (2003) stated self-transcendence helped a person make sense of living, aging, and dying by providing meaning and sustaining well-being and ego-integrity. The theory of self-transcendence involved interactions among three major concepts: vulnerability, wellbeing, and self-transcendence. Self-transcendence was not only the result of life experience and patterns of behavior accumulated over the lifespan but more importantly might be evoked by an acute loss or illness, creating a sense of vulnerability and an acute awareness of mortality. Self-transcendence then functioned by influencing the ways individuals perceived challenges, choices, and their own abilities and priorities, with the potential for well-being as the outcome. Well-being was marked by life satisfaction, positive self-concept, hopefulness, and a sense of meaning in life.

Development of the theory of self-transcendence (Reed, 1991a, 2003) included not only a theoretical basis, but was also based on nursing practice and multiple concurrent empirical testing of two propositions (Reed, 1991a): a) self-transcendence was greater when end-of-life issues, i.e., vulnerability, were confronted; and b) selftranscendence was positively related to well-being. The notion of a spiritual perspective, as an indicator of self-transcendence, as one way of expanding self-boundaries was examined in two studies of adults facing end-of-life issues, to test the first proposition (Reed, 1986a, 1987). The first was among 114 ambulatory adults, half with incurable 
cancer (1986a), and the second (1987) among 300 adults in three groups (100 terminally ill; 100 non- terminally ill hospitalized adults; 100 well non- hospitalized adults). Spirituality, an indicator of self-transcendence, was defined as prayer, meditation, forgiveness, and belief in God or a higher power and measured using the Spirituality Transcendence Scale. Both studies supported the proposition of the self-transcendence theory, that nearness of the end of life increased spirituality, while controlling for age, gender, education, religion, or hospitalization. A significant positive relationship was also found between spirituality and well-being in the terminally ill groups.

Another set of correlational and longitudinal studies tested the second proposition among middle-old and oldest-old adults, aged 60 to 100 (Reed, 1986b; 1989a; 1991b). Using the Self-transcendence Scale (STS) (1989b) to measure psychosocial indicators of self-transcendence, such as sharing wisdom with others, helping others in some way, and finding meaning in past experiences. Reed found a negative correlation between depression and self-transcendence, and self-transcendence was found to be a significant predictor of future depression. A qualitative study used content analysis to identify self transcendence as a developmental factor participants felt led to subsequent hospitalization for depression. A study focused on the oldest-old, aged 80 to 100, also found a negative correlation between self-transcendence and depression, with qualitative data suggesting four self-transcendent patterns: inner directedness, generativity, temporal integration, and body transcendence (Reed, 1991b).

These empirical studies validated Reed's theory (Reed, 1986b; 1989a; 1991b). Other studies extended the theory of self-transcendence to different samples, such as individuals facing serious illness (AIDS) or end-of-life issues (Coward, 1990, 1991, 
1995, 1996) and healthy adults aged 19 to 85 (1996), and 107 health older adults

(Walton, Shultz, Beck, \& Walls, 1991). Of particular interest to the present study, Upchurch (1999) found a significant positive relationship between self-transcendence and daily functional ability (ADLs) in 88 chronically ill older adults. Upchurch suggested the relationship might help explain why some older adults were able to adapt to aging and maintained their independence while others did not, even while controlling for health status.

Operationalized Definition of Transcendence

Similar to Tornstam's (1989) theory of gerotranscendence, Reed (1991a, 2003) proposed that the impulse toward self-transcendence was a developmental necessity, inherent in being human, that demanded expression, although Tornstam saw gerotranscendence as an abrupt shift to a qualitatively new reality, while Reed viewed self-transcendence as a gradual, nonlinear process of development much like other developmental stages. Tornstam based the gerotranscendence theory, developed specifically for older adults, in disengagement theory, although arguing that the withdrawal associated with gerotranscendence was not a passive process, but a turn toward an active inner life. Tornstam's theory placed greater emphasis on a need for solitude than self-transcendence theory. Reed's self-transcendence theory, developed among end-of-life populations such as older adults and the terminally ill, was also based in life span development theory, but added ideas from Rogers' (1970) model of conceptual boundaries.

The gerotranscendence theory (Tornstam, 1989) had limited empirical support with inconsistent findings, while the theory of self-transcendence was empirically 
validated and extended to a number of other end-of-life populations. Reed's theory (1991a, 2003) appeared to be somewhat more holistic than Tornstam's, more concrete and rooted in reality, and it specifically focused on the dimension of health and illness. Life satisfaction and well-being were the primary outcomes of successful aging achieved through both transcendence theories. A significant practical difference in the theories was in their level of abstractness and testability. Reed's theory, as a mid-range nursing theory, was concrete, parsimonious, and easily operationalized, while the theory of gerotranscendence was abstract and complex.

Given the congruence of self-transcendence (Reed, 1991a, 2003) with the concept of gerotranscendence used by Flood (2005) in the theory of successful aging, the lack of a validated English-translation of the gerotranscendence scale, and the well-established validity of Reed's self-transcendence theory with a well-tested instrument, transcendence was operationalized for the study as self-transcendence.

The theoretical background for the final major construct of Flood's theory, i.e., successful aging, was identified and organized within three major perspectives, i.e., the biomedical perspective, the psychosocial perspective, and the perspective of older adults themselves. The new mid-range nursing theory of successful aging (Flood, 2005) was described and analyzed, evaluating the adequacy of the Flood's theory to generate research, guide clinical practice, and support effective policy development to increase positive aging and quality of life for older adults. Finally, results of a feasibility study which explored methods and methodology for future exploration of Flood's theory were summarized. 


\section{Successful Aging}

The final construct in Flood's theory (2005) was successful aging. Review of the literature revealed there was no consensus on definitions of successful aging, the conditions that fostered success, or outcomes of the phenomenon (Bowling, 2007; Bowling \& Dieppe, 2005; Bowling \& Iliffe, 2006; Phelan, et al., 2004). Few studies examined the meanings given to successful aging by older adults themselves (Phelan \& Larson, 2002; Strawbridge, et al., 1996), nor was a spiritual or transcendent dimension considered (Sadler \& Biggs, 2006). An examination of successful aging in multiple contexts was conducted to clarify its meaning and to explore its potential as a guide to improving quality of life among older adults. To gain a comprehensive understanding of the origins, uses and meanings of the construct of successful aging, the literature was organized into three categories, representing the primary philosophical perspectives on successful aging: biomedical views of successful aging, psychosocial views, and finally the views of older adults. Theoretical and empirical literature within each category was examined. Subsequently, a new theory of successful aging (Flood, 2005) which merged all three perspectives, was described and analyzed.

\section{Biomedical perspectives on successful aging.}

In public health and epidemiology, decreased morbidity and mortality were common outcomes; success was defined as being alive (Rowe \& Kahn, 1987, 1997; von Faber, et al., 2001). Successful aging studies focused on long term benefits of research in behavioral determinants of health and genetic/biochemical influences on aging, in populations from childhood to late adulthood. In medicine, congruent with the tendency to focus on disease rather than health, success meant the absence of disease and 
disability, independent cognitive and physical function, and productive activity (Rowe \& Kahn, 1987, 1997). The presence of disease excluded success, despite the World Health Organization (WHO) definition of health as a state of complete physical, mental, and social well-being and not merely the absence of disease (WHO website, 1946).

A review of empirical studies determined that the most common definition of successful aging (Depp, Glatt, \& Jeste, 2007; Depp \& Jeste, 2006; Peel, Bartlett, \& McClure, 2004) was Rowe and Kahn's, “The avoidance of disease or disability, the maintenance of high physical and cognitive function, and sustained engagement in productive activities" (1997, p. 433). Rowe and Kahn's theory of successful aging was developed in conjunction with the MacArthur Foundation Study of Successful Aging, a ten year, \$20 million multi-site longitudinal study that investigated physical and cognitive function among a volunteer sample $(n=4,030)$ of healthy, high-functioning older adults (National Institute on Aging, n.d.).

Results of the MacArthur aging studies led to a new attitude toward aging, labeled the New Gerontology (Holstein \& Minkler, 2003). This paradigm change shifted the view of old age from inevitable senescence and decline to the potential for extended health and activity well into old age, drawing attention to the fact that only a small number of people ended their lives in nursing homes and many older adults remained healthy and active in late life (Gordon, 1998). The shift led to numerous intervention studies designed to identify, prevent, and reverse functional losses associated with usual (non-pathological) aging (Scheidt, Humphreys, \& Yorgason, 1999; National Institute on Aging, n.d.).

Major findings of the MacArthur studies identified protective factors associated with physical exercise, participation in social activities or groups, and a positive mental 
attitude (National Institute on Aging, n.d.). Results included that both strenuous and moderate physical activity were equally effective in maintaining function in older adults, due to increased muscle strength and confidence, and resulted in decreased depression. The MacArthur studies clarified that social support could be either emotional (positive benefit) or instrumental (potentially negative when independences was lost). Studies also identified strategies that maintained mental acuity to delay or avoid age-related dementia (Gordon, 1998). Even older adults who already had some level of decline or mild chronic disease experienced beneficial effects from increasing physical activity and socialization (National Institute on Aging, n.d.).

Criticism of the MacArthur studies centered on a number of concerns: a) people were made accountable for their own success, regardless of physical and mental disabilities or socioeconomic, cultural, or environmental limitations; b) the study ignored the heterogeneity in the population, and the lack of generalizability from a group of exceptionally healthy older adults to the total older adult population; c) the study suggested a causal rather than a correlational relationship, despite the epidemiological, longitudinal nature of the study; d) use of a volunteer sample made results subject to serious self-selection bias; and e) other positive factors in older adults with chronic disease or limited function which contributed to quality of life were not considered (Scheidt, et al., 1999).

Another criticism, related to the negative connotation of the term success, was related to the perception that success was fully within the control of individuals. The fundamental injustice of failing to consider gender, racial, and economic factors unfairly excluded older adults with existing chronic disease or disability of any degree, as well as 
groups with limited opportunities for success, such as women, racial and cultural minorities, and the lower socioeconomic group. Common attitudes toward success and failure in the U.S. culture prompted a "blame the victim" mentality for older adults who failed to succeed, suggesting they therefore somehow merited the negative consequence (Holstein \& Minkler, 2003; Schulz, \& Heckhausen, 1996). Kahn (2002) addressed these concerns about the materialistic, competitive connotations of successful aging. In a guest editorial in The Gerontologist; Kahn stated, "Successful aging means just what it says aging well, which is very different from not aging at all" (p. 725). Kahn shared the concern about the interpretation of the term but attributed the responsibility to the current American culture rather than something intrinsic to the concept.

Beyond a concern for justice and quality of life among older adults, Knickman and Snell (2002) pointed out the economic consequences to society at large of failure to implement structural changes necessary to accommodate the population bulge of the baby boom birth cohort. Effective actions proposed by Knickman and Snell included focusing research on not only behavioral but also social and environmental determinants of health, addressing problems with the health care system, developing innovative community programs and services, and reforming social and economic policies at the local, state and national levels. The potential magnitude of the problem might be understood by realizing that over 60 percent of current adults, ages 50 to 64 , have been diagnosed with at least one chronic health condition, such as arthritis, cancer, diabetes, heart disease, high cholesterol, or hypertension (Collins, Davis, Schoen, Doty, \& Kriss, 2006). Excluding large numbers of older adults from the potential for a positive old age, as defined in the biomedical model, did nothing to identify other potentially effective methods of 
improving quality of life for older adults, including those with chronic disease, while protecting society from an overwhelming burden.

Despite methodological concerns and disparities in opportunities for success, the benefits derived from biomedical studies of successful aging appeared indisputable. As a result of the redefinition of aging, attitudes toward aging greatly shifted from a decremental perspective on aging (Reed, Shearer, \& Nicoll, 2004) to a more positive, and realistic view, identifying effective changes in behavioral risk factors which generated a significant increase in aging research and were of unarguable benefit.

Psychosocial perspectives on successful aging.

In sociology, successful aging was viewed as a transaction between society and the individual, related to environmental, social, and cultural factors (Fentleman, 1990); optimal overall functioning and well-being (von Faber, et al., 2001); or satisfaction with reaching individual goals (Havighurst, 1963; Keith, Fry, \& Ikels, 1990). In psychology, successful aging was considered both a process of continual adaptation (Baltes \& Baltes, 1990; von Faber, et al., 2001), and a state, i.e., a better than normal state of being old (von Faber, 2001).

The majority of psychosocial definitions of successful aging focused on successful aging as a process of adaptation to age-related changes, based on goal management (Baltes \& Baltes, 1990), development and maintenance of control-related processes (Schulz \& Heckhausen, 1996), and proactive adaptation (Kahana \& Kahana, 1996). An example of a well-established theory of successful aging from the psychosocial perspective, which was supported by multiple empirical studies, was offered by Vaillant and Mukamal (1990, 2001; Vaillant, 2002). Vaillant and Mukamal (1990) 
studied successful aging in the Harvard Study of Adult Development ( $N=569)$, a prospective, longitudinal study that followed two socially diverse cohorts of adolescent men (a college cohort and a core-city cohort) over 60 years, offering a heuristic model of successful aging. Based on the results of the study, Vaillant and Mukamal (1990) classified aging along a continuum extending from the happy-well to the sad-sick. Three dimensions of aging were reported: decline, change, and development. Decline was explained by Vaillant and Mukamal, as fundamentally opposite of success, inherently negative. Change was described as a relatively neutral dimension of aging, exemplified by changes in hair color, waistlines, crow's feet, and the frequency of lovemaking, emphasizing that the ability to love and be loved did not diminish in old age. Development, the last dimension of aging compared late life maturity to a fine wine. Older adults were still capable of development in late life, and were more patient, tolerant, and accepting of their own feelings as well as others' feelings. Moreover, older adults were more likely to accept paradox, appreciate relativity, and understand that how the present is related to both the past and the future. Within these three dimensions, six domains of function placed participants along the continuum from happy-well to the sadsick: a) objective physical health; b) subjective functional ability; c) length of active life; d) objective mental health; e) subjective life satisfaction; and f) social support.

Lay perspective on successful aging.

Major problems among successful aging studies in all disciplines were pointed out by Vaillant and Mukamal (2001). The problems contributing to confusion on the meanings, predictors, and criteria for successful aging were: researcher bias in selecting criteria and indicators of the construct; the number and complexity of relationships 
among predictors; confusion about causal direction; change over time in the relative importance of predictors; and the limits of self-report measures.

An issue involved in both biomedical and psychosocial models of successful aging was who should determine criteria for success. While definitions and normative criteria developed by researchers provided concrete, measurable standards that could be evaluated and tracked over time and among populations, external norms missed the heterogeneity between and within older adults, did not account for social contexts in which aging occurs, and ignored the potential for multiple outcomes and diverse standards of success (Baltes \& Carstensen, 1996). Many more people considered themselves as successful-agers than were identified in empirical studies, evidencing the discrepancy in criteria (Phelan, et al., 2004).

Strawbridge and colleagues (2002) reported results of a study that compared criteria for successful aging identified by 867 Alameda County Study participants, aged 65-99 years with the Rowe and Kahn definition $(1987,1997)$. While $81.2 \%$ of older adults were excluded according to the Rowe and Kahn definition, $50.3 \%$ of participants considered themselves successful agers, despite the presence of chronic diseases and functional limitations. Poon, Gueldner, and Sprouse (2003) argued that successful aging should be defined not by the absence of disease but rather the way people cope with disease.

This perspective was reflected in a study on successful aging that considered freedom from disease and disability important, but not essential, components of successful aging, and focused on how older adults actually coped with aging and disease. According to Bowling and Iliffe (2006), in a cross-sectional study among 999 adults aged 
65 and older, criteria for successful aging were identified for three models of aging. Biomedical models almost exclusively used Rowe and Kahn's $(1987,1997)$ definition of successful aging. Psychological models of successful aging produced criteria for successful aging such as self-worth, a sense of control, self-efficacy, effective coping strategies, adaptation and goal achievement. While these criteria allowed for success, regardless of physical health and functioning, additional criteria were identified by older adults, in what Bowling and Iliffe termed the lay model. Previously unidentified social and behavioral determinants of health that contributed to successful aging were income, perceived social resources, and ratings of the physical environment (e.g. transport, closeness to shops, services), and environmental conditions (e.g. crime, vandalism, graffiti, traffic, air quality), and walkability, that is a pleasant and safe destination.

Summary of criteria and indicators of successful aging.

A number of other studies identified indicators or outcome criteria of successful aging, including: a) health, functional status, and activity (Rowe \& Kahn, 1987, 1997); b) life satisfaction (Bowling, 2007; Bowling \& Dieppe, 2005; Neugarten, Havighurst, \& Tobin, 1961); c) emotional well-being (Freund \& Baltes, 1998, 2002a; Lawton, 1975, 2003; Ryff, 1989; von Faber et al., 2001); d) quality of life (Power, Quinn, Schmidt, \& the WHOQOL-OLD Group, 2005; Saxena, Orley, \& the WHOQOL Group, 1997; WHOQOL-BREF Group, 1998); e) purpose in life (Adler, 1997; Fisher \& Specht, 1999); f) autonomy or control (Schulz \& Heckhausen, 1996); and g) spirituality (Sadler \& Biggs, 2006).

There was also debate about what conditions fostered or predicted successful aging (Bowling \& Dieppe, 2005; Phelan, et al., 2004). Factors associated with successful 
aging included health behaviors, e.g., physical activity, smoking cessation, and avoidance of obesity (Rowe \& Kahn, 1987, 1997; Seeman, Unger, McAvay, \& Mendes de Leon, 1999; Vaillant \& Mukamal, 1990); personal and environmental factors, e.g., culture, race, age, education, marital status, and social network (Holstein \& Minkler, 2003; Sadler \& Biggs, 2006; Vaillant \& Mukamal, 1990); social support ( Fry, 2003; Gavan, 2003; Strawbridge, et al., 1996); adaptation or coping (Aspinwall \& Taylor, 1997; Baltes \& Baltes, 1990, Baltes \& Carstensen, 2003; Greenglass, 2002; Institute of Medicine, 2006; Kahana, et al., 2002; Ouwehand, de Ridder, \& Bensing, 2007; Roy \& Andrews, 1999; Schwarzer \& Taubert, 2002); self-efficacy (Bandura, 1997; Pearlin, 1999; Lang, Featherman, \& Nesselroade, 1997; Seeman, et al., 1999); motivation or control (Schulz \& Heckhausen, 1996); perceived health (Bowling, Fleissig, \& Gabriel, 2003); spirituality ( Kirby, Coleman, \& Daley, 2004; Sadler \& Biggs, 2006); and transcendence (Erikson \& Erikson, 1997; Flood, 2005; Maslow, 1968, 1971; Reed, 2003; Tornstam, 1989). A midrange nursing theory of successful aging argued that adaptation and transcendence together significantly contributed to success (Flood, 2005).

While there was confusion about definitions and measurement, successful aging was a widely used construct that provided a potential meeting ground for multidisciplinary efforts to improve quality of life for older adults. Glass (2003, p. 382) argued that a synthesis of both the biomedical and the psychosocial perspectives was badly needed.

\section{Mid-range Nursing Theory of Successful Aging}

A mid-range nursing theory of successful aging (Flood, 2005) was proposed which bridged the gap between the biomedical and psychosocial domains. The theory 
also added the perspectives of lay older adults, allowing for the importance of social and behavioral determinants of health as well as existential or spiritual factors identified in the literature. The definition of success in the theory addressed concerns regarding exclusion of older adults with disease or disability, as well as disparities due to gender, race, culture, or socioeconomic status. Finally, Flood's Theory of Successful Aging was described, analyzed, and evaluated.

\section{Description of the Successful Aging Theory}

Flood (2003, 2005) defined successful aging as an individual's accomplishment of goals that were perceived as effective in adapting to the physical and functional changes accumulated over time, while experiencing spiritual connectedness and a sense of meaning or purpose in life. The successful aging theory (SAT) (Flood, 2005) proposed a set of coping processes, used to achieve adaptation, which in turn resulted in successful aging. Flood derived three coping mechanisms from Roy's theory (1984; Roy \& Andrews, 1999): functional performance; intrapsychic factors; and spirituality. A fourth coping mechanism, derived from Tornstam's theory (1997a), was activated only when adaptive coping through Roy's coping mechanisms had successfully fulfilled basic needs for survival, growth, and mastery. This final coping mechanism was gerotranscendence, a major shift in the person's worldview, where a person examines one's place within the world and in relation to others. A model of the SAT in Figure 1 serves to clarify the relationships among these constructs.

The SAT (Flood, 2005) was multidimensional, operating in the physical, functional, spiritual, and psychological domains, which were reflected in theoretical definitions for the coping processes (p. 38): Functional performance coping mechanisms 
involved consciously selecting goals to adapt to accumulated physical and functional losses associated with aging. Intrapsychic factors or psychological coping mechanisms involved innate and enduring features of an individual's character that could either support or inhibit the individual's ability to adapt and problem solve. Spirituality incorporated personal views and behaviors arising from connectedness to a higher power, a search for the sacred in feelings, thoughts, experiences, and behaviors. Finally, gerotranscendence was a major shift in worldview from a materialistic and rationalistic perspective to a more mature and existential one, that was an inherent part of late life development. Flood's (2005) theory merged multidimensional coping strategies from Roy's (1984) adaptation model with Tornstam's (1989) view of transcendence as a shift in perspective, resulting in a new perspective on successful aging.

According to (Flood, 2005), examples of input stimuli which would trigger functional performance coping mechanisms were a diagnosis of a new disease or a change in functional ability while potential outputs might be reflected in health promotion activities, physical health, or physical mobility. Output responses of intrapsychic coping processes, such as creativity, low levels of negativity, and a sense of personal control, might be stimulated by the same diagnosis of disease or by growing feelings of loss. Output responses to spiritual coping included increased religiosity and/or a deepening awareness of the role of spirituality in one's life. Adaptive coping skills in these first three domains then triggered a transcendent shift in reality. Subsequently, outputs of gerotranscendent coping were decreased death anxiety, increased engagement in meaningful activities that were focused on personally valued activities and relationships, while devoting less energy to superficial relationship or activities; 
increased self-acceptance, decreased concern for the judgments of others; and increased wisdom. Flood noted coping processes might act directly or could serve as stimuli for responses in other coping mechanisms.

Analysis of the Theory of Successful Aging

Several features of Flood's (2005) theory differed from other successful aging theories. The major contrast between Rowe and Kahn's $(1987,1997)$ perspective on successful aging and that of Flood centered on the definition of success. Flood's definition allowed for assessment of success judged by the individuals own satisfaction with accomplishment of goals, based on personal values and priorities. This was important because use of externally-imposed criteria for success and normative standards may not identify issues of importance to large numbers of older adults, resulting in an incomplete image of successful aging. Flood's definition also added a multidimensional and holistic perspective not identified in other theories identified in the review of the literature. This perspective appeared uniquely suited to nursing's holistic values (HansenKyle, 2005), addressing concerns of traditional importance to the profession. Five specific factors were recognized which related to issues identified in the literature.

First, by basing the SAT in Roy's (1984) adaptation model (RAM), Flood (2005) merged issues of mortality and morbidity from the biomedical/public health view of successful aging (Rowe \& Kahn, 1987, 1997) with psychosocial perspectives on coping and adaptation (Aspinwall \& Taylor, 1997; Baltes and Baltes, 1990; Schwarzer \& Taubert, 2002). The RAM specified adaptive behaviors within physiologic/physical, role function, interdependence, and self-concept/group identity domains. 
Second, the addition of the construct of transcendence (Flood, 2005) complemented and completed Roy's (1984) spiritual domain, adding a life span development framework. The optimal outcome of adaptation, according to Roy, was integrity, congruent with the construct of transcendence. Transcendence incorporated existential and transcendent values that were a product of developmental maturity, such as self acceptance, concern for others, especially the next generation, a sense of meaning and purpose in life, and ego-integrity or oneness with the self, other, and a higher power (Maslow, 1969, 1971; McKee \& Barber, 2001; McFadden, 1996; Reed, 2003; Sadler \& Biggs, 2006; Thibault, Ellor, \& Netting, 1991; Thomas, 2004; Tornstam, 1989, 1996, 2005).

Third, the addition of this dimension brought the SAT in line with values and priorities expressed by older adults in studies that investigated successful aging by asking older adults what they considered success, instead of imposing external norms (Bowling, 2007; Bowling, \& Dieppe, 2005; Bowling, \& Iliffe, 2006; Fisher, \& Specht, 1992; 1995, 1999; Moody, 2002; Phelan, et al., 2004; Runquits, \& Reed, 2007; Strawbridge, et al., 2002; Strawbridge, et al., 1996; Wadensten, \& Carlsson, 2005; Wong, 1998).

Fourth, the criteria for success in the SAT (Flood, 2005) were non-normative, varying both between and within individuals and changing over time and context, which accounted for the observed heterogeneity among older adults. Unlike normative criteria, the older adult's own perspective demanded criteria defined by the individual's personal goals, values, and resources. In the SAT, "success" was based on the individual's perceived satisfaction with reaching their own goals, in domains of importance to themselves. As Baltes and Carstensen (1996, p. 399) explained, 
"We cannot predict what any given individual's successful aging will look like until we know the domains of functioning and the goals that that individual considers important, personally meaningful, and in which he or she feels competent."

Finally, concerns about issues of social justice, cultural sensitivity, and an individual's right to autonomy and self-determination (Angel \& Angel, 2006; Austin, 1991; Bearon, 1996; Gilhooly, 2007; Holstein, \& Minkler, 2003; Knickman, \& Snell, 2002; McInnis-Dittrich, 2005) were considered. Flood's (2005) theory allowed for a favorable outcome to aging, even in the presence of chronic disease, limited physical or cognitive function, age, gender, or restricted cultural or socioeconomic resources.

\section{Evaluation of the Theory of Successful Aging}

Flood (2005) identified a need for a holistic nursing theory to guide nursing practice (Wadensten \& Carlsson, 2003) and to generate propositions for research to further refine the theory and expand it to larger samples and additional populations (Flood, personal communication, February 22, 2008). The theory of successful aging (SAT) was a mid-range nursing theory (Flood, 2005), developed through the process of deductive reformulation (Reed, 1991a), a process integrating existing knowledge from a non-nursing theory (Tornstam, 1996) with established nursing theory (Roy, 1984). As a mid-range theory, the purpose of the SAT was prescriptive, with an appropriate level of abstraction and scope, and a limited number (5) of relatively concrete concepts and propositions (McEwen \& Wills, 2002; Meleis, 2007). Concept analysis was used to develop conceptual definitions which were congruent with literature and theory. Propositions specified causal relationships among the concepts, based on explicitly stated 
assumptions. A well-designed model linked the concepts, which were operationally defined, appropriate for empirical testing, and used well-validated instruments. Terms for concepts, assumptions and propositions were used consistently and supported a logically organized purpose which was significantly related to nursing practice.

The SAT (Flood, 2005) addressed a phenomenon congruent with nursing paradigms and values, i.e., an increased older adult population with a different set of challenges that required a different pattern of nursing response. The theory had the potential to guide nursing practice by facilitating development of clinically significant, feasible, low cost interventions, was relevant to current economic and political issues, and was socioculturally sensitive. Internal validity of the theory appeared adequate and appropriate to a mid-range theory, on the basis of clarity, consistency, and level of theory development. The SAT proceeded logically from an existing theoretical basis; concepts and propositions were linked to the theoretical foundation, as demonstrated in a model of the relationships among the concepts. See Figure 1.

It was noted that both foundational theories selected by Flood (2005) were quite abstract and not easily accessible due to complexity and vocabulary. The adaptation theory (Roy, 1984) was not based in a life span development perspective, did not consider the unique needs of older adults, and lacked a measurement instrument validated in an older adult population. The conceptual validity of the gerotranscendence theory (Tornstam, 1989, 1996, 2005) was unclear. The theory had not been used outside of a narrow range of Scandinavian countries, and no examples of use of an English-language version of the investigator-developed instrument were identified (Jönson \& Magnusson, 2001). Conceptual validity might be strengthened if the successful aging theory were 
founded on adaptation and transcendence theories that were more concrete, with validated instruments, and evidence of empirical testing in a population of older adults. Operational Definition of Successful Aging: Successful Aging Inventory

While conceptual validity for successful aging theory (Flood, 2005) was supported by theoretical and empirical literature, as well as by previous concept analysis (Flood, 2003), a subsequent study conducted by Flood (2008) to operationalize successful aging, increased internal validity. Flood used principal component analysis to develop the Successful Aging Inventory (SAI) which identified two factors that accounted for $47.78 \%$ of the variance: coping/adapting (36.4\%), and existential (11.38\%), which were congruent with the theory. Convergent validity was established by correlation of the SAI with the Purpose in Life Test (PIL) (Crumbaugh \& Maholick, 1969) $(p<0.000, r$ $=0.512)$; and Pearlin and Schooler's (1978) Mastery Scale $(p<0.000, r=0.395)$. Divergent validity was demonstrated by an inverse correlation with the Center for Epidemiologic Studies Depression Scale (CES-D) (Radloff 1977) $(p<0.001, r=-0.338)$. A concern with Flood's (2008) SAI was the failure of the Likert version of the SAI to correlate significantly with the Life Satisfaction Inventory-A (LSI-A) (Neugarten, Havighurst, \& Tobin, 1961). While life satisfaction was a frequently used indicator of successful aging in other studies, a wide variety of scales were used to measure life satisfaction, thus it was possible convergent validity might be found using another instrument to measure life satisfaction. Flood reported it was possible the relationship between the SAI and the LSI-A lacked significance due to the wording of at least two items on the LSI-A (electronic communication, February 24, 2009). For example, a negative response to, "I feel my age but it does not bother me," might indicate someone 
feels younger than their age; they would disagree with the statement. Scoring on the LSIA would interpret the response as a negative response, which would be scored a 0 for the item. Another item referred to, "Man's lot in life," which could be interpreted literally as referring to men but not women. It was also possible, according to Flood, that failure to find significance for the LIS-A was related to sample size and lack of power. The sample size used by Flood for instrument development was minimally acceptable for the 20 itemSAI, but with the addition of the other scales, a larger sample was required for adequate the power. Considering the sample size, the significant relationships with the PIL, Mastery Scale and CES-D that were observed reflected positively on the strength of the correlations and the validity of the scale.

External reliability and generalizability of the SAI were also examined. While reliability of the SAI was evidenced by a Chronbach's alpha of .88 , a limitation of Flood's (2008) instrument development study was the lack of a test/re-test statistic supporting stability of the SAI.. Flood's instrument development study used a nonrepresentative convenience sample, introducing the potential for self-selection bias and limiting generalizability. The volunteer sample was recruited at a local senior citizens center among a population of community-dwelling Southern older adults. Age ranged from 52-100 with a mean of 75.01 (S.D. 8.24). Eighty-nine percent of participants were white, two-thirds were women, and $45.5 \%$ were married, with only $34.5 \%$ widowed. Higher age groups of older adults typically consisted of more women than men, and a larger proportion of widowed participants, but the relationship of age with marital status could not be assessed without knowing the ages of the participants in the sample. Mean 
education was 12.87 years and $66.5 \%$ reported having an income that allowed them to "live comfortably," while $20.5 \%$ felt they "barely got by" (Flood, 2008).

The potential for generalizability of the instrument to other samples from the same populations and to slightly varying populations was also a limitation of the SAI. While generalizable results should be sufficiently robust to be used in slightly varying formats, a dichotomous version of the SAI failed to find significance and Cronbach's alpha was only .56 (Flood, 2008).

Feasibility Study

A feasibility study based in Flood's (2005) successful aging theory was recently completed by the investigator (McCarthy, 2009), in preparation for the present dissertation study. The feasibility study was a descriptive, cross-sectional mixed-method study $(\mathrm{N}=20)$ conducted to examine potential methods, including data collection and measurement instruments, that might be used to explore Flood's (2005) Theory of Successful Aging. The sample was a convenience sample of non-demented older adults, aged 65 and older, residing in a middle-income independent and assisted living continuing care retirement community (CCRC). Research questions focused on the feasibility of administering survey instruments to participants in small groups $(n=5)$, the usefulness of the Mini-Cog Test to screen for dementia, potential rationales to select survey instruments to measure the two independent variables, transcendence and adaptation, evidence of the validity and reliability of a new instrument, the SAI, developed by Flood (2008) to measure the single dependent variable, successful aging, and the usefulness of an investigator-developed instrument to obtain a) demographic data; b) dichotomous responses (yes/no) to a question on whether the respondent feels he 
or she is aging successfully; and c) qualitative data from responses to the single openended question, "What does successful aging mean to you?"

Results of the study (McCarthy, 2009) supported the feasibility of using investigator-administered survey questionnaires for small groups, the Mini-Cog test functioned as intended, and instruments were selected for each major variable: the $15-$ item Self-transcendence Scale (STS) (Reed, 2003), a 14-item proactive coping subscale (PCS) (Greenglass et al., 1999), and the 20-item Successful Aging Inventory (SAI) (Flood, 2008) as an outcome measure. A model regressing successful aging (SAI) on self-transcendence (STS) and proactive coping (PCS) was statistically significant $(R=.60$, $p=.03$ ) and explained $35.5 \% \%$ of the variance. The investigator-developed instrument was useful to obtain sociodemographic data. All responses to the question, "Do you feel you are aging successfully?" were positive. The open-ended question, "What does successful aging mean to you?" produced rich descriptive data, related to five themes: a) activities, b) people, c) resources, d) limitations, and e) attitudes.

In general, the feasibility study (McCarthy, 2009) supported the methodology proposed to explore the theory of successful aging (Flood, 2005). Administration of surveys to small groups appeared to be a feasible option, with some adjustments. Selected questionnaires required a limited number of items, thus decreasing respondent burden, with good potential to find significance in the study. The quantitative question on participants' perceived success in aging was retained, despite dichotomous responses that were all positive, as a larger sample in a different setting may demonstrate more variance. The open-ended question was eliminated, as the depth and scope of the qualitative data 
suggested that this method merits further investigation beyond the scope of the proposed study.

\section{Summary of the Review of the Literature}

The review of the literature explored theoretical support and empirical uses of three constructs: transcendence, adaptation, and successful aging. The Theory of Successful Aging (Flood, 2005) could make a unique contribution to aging theory by merging the biomedical and psychosocial models with the perspectives of older adults, using non-normative criteria. The new successful aging theory incorporated a spiritual and transcendent dimension not considered in other aging theory. Finally, successful aging, as defined by Flood, addressed important issues of social justice, cultural sensitivity, and an individual's right to autonomy and self-determination. The dissertation study aimed to explore the potential usefulness Flood's (2005) Theory of Successful Aging as a guide to nursing practice, research, and policy.

The next section of the dissertation discusses the purpose of the study, specific research questions, and methods used to conduct the study. 


\section{CHAPTER III}

\section{METHODS}

This chapter describes the methods used to explore the Theory of Successful Aging (Flood, 2005), including the purpose of the dissertation study, specific research questions, and the study design selected to address the questions. Conceptual and operational definitions of study variables are provided, with a table of variables displayed in Table 1 on page 17. A model of the relationships among the variables is presented in Figure 2. The sample and setting for the dissertation study; measurement instruments used; and methods for data collection, management, and analysis are detailed. Ethical considerations and anticipated limitations of the study are also described.

\section{Study Design and Purpose}

A descriptive cross-sectional, correlational study (Shadish, Cook, \& Campbell, 2002) explored Flood's (2005) Theory of Successful Aging. The study examined relationships among two independent variables, transcendence and adaptation, with a single dependent variable, successful aging, controlling for age, income, health, and functional status. The stratified random sample $(\mathrm{N}=112)$ consisted of non-demented, low-income, racially diverse older adults, aged 65 and above, living independently or receiving assisted living 
services in a 390-unit, non-profit continuing care retirement community (CCRC). The CCRC used as the setting for the study provided both Figure 2

Figure 2

Adapted Successful Aging Model

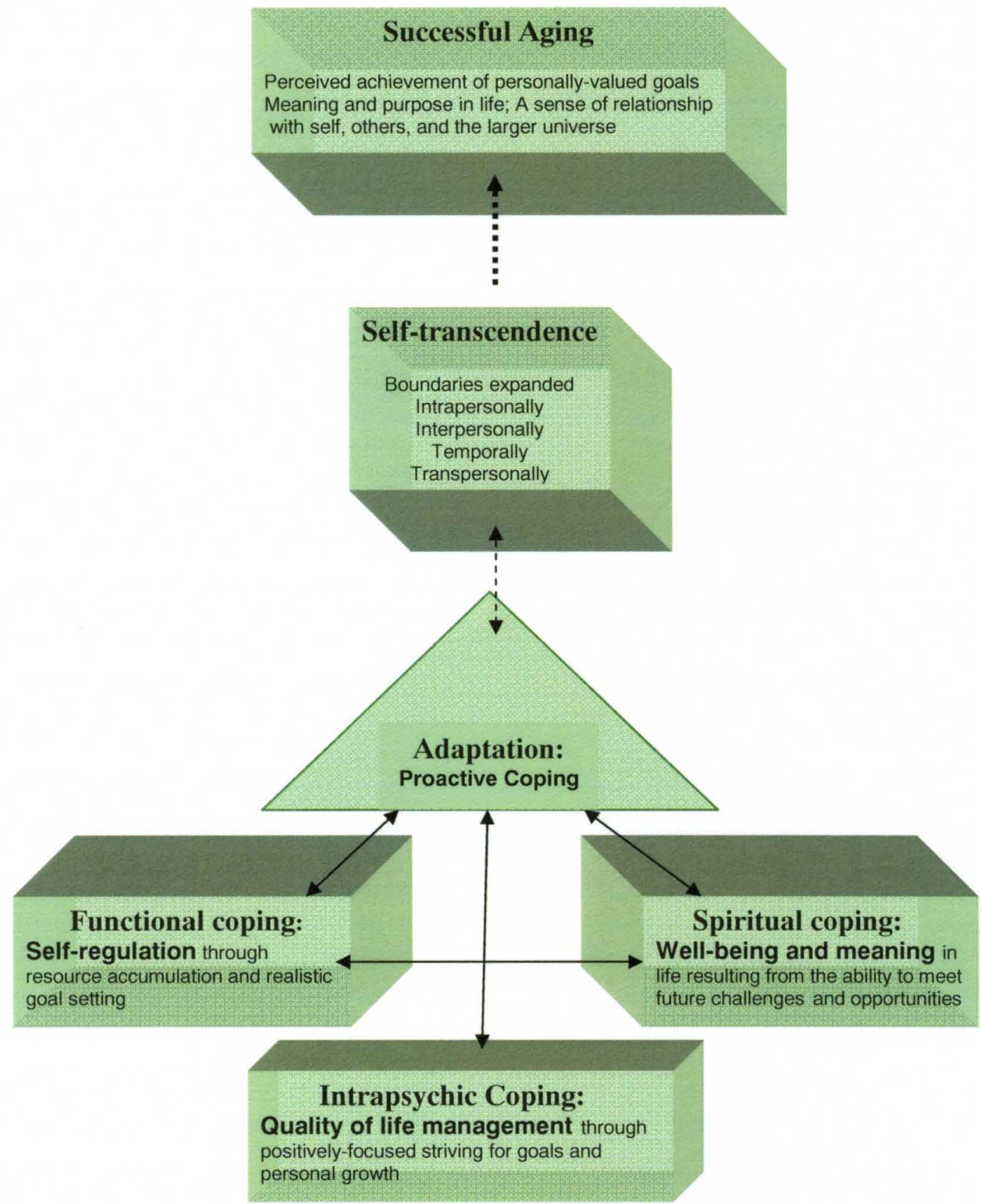


independent living housing and assisted living services, was operated by a charitable religious organization and was located in an urban county of a Southern city. Quantitative data was obtained using an investigator-administered set of validated questionnaires with small groups (5 to 7 participants). An investigator-developed questionnaire was also used to obtain: (a) socio-demographic data (age, gender, race, marital status, education; (b) perceived income level, (c) independent or assisted living status, (d) self-reported perceived health, (e) number of chronic diseases; and (f) a dichotomous response (Yes/No) to a single question, "Do you consider yourself to be successfully aging?" Data were also obtained from a sampling frame provided by the CCRC, specifically income, indicated by one of three HUD rent levels associated with income, and functional status, indicated by either independent or assisted living status. A model of the relationships among variables in the study, adapted from Flood's (2005) model, is displayed in Figure 2. It should be noted that specific components of the Proactive Coping Scale (Greenglass, 1999), used to operationalize adaptation for the present study and incorporated in the adapted model, are congruent with the coping strategies used by Flood, based on the RAM (Roy, 1984), to describe the concept of adaptation. Similarly, propositions of the Self-transcendence theory (Reed, 2003) used in the adapted model are congruent with the propositions of gerotranscendence theory (Tornstam, 1986, 2005) used by Flood in the original model. 


\section{Research Questions}

1. What are the differences in successful aging, if any, between groups based on age, gender, race, marital status, education, income, function, perceived health, or number of chronic diseases?

2. What are the relationships among adaptation, transcendence and successful aging? Are both adaptation and transcendence predictors of successful aging?

3. To what degree do adaptation and transcendence explain any observed variance in successful aging among participants in the target population?

4. What are the differences in adaptation, transcendence, and successful aging between participants who report they are aging successfully and those who do not?

Sample and Setting

Description of the population and the sample.

The target population of interest was non-demented older adults, aged 65 and older, living independently or receiving assisted living services in a 392-unit, low-income continuing care retirement community (CCRC) in an urban county of a mid-sized Southern city. Table 2 displays national, state, and local demographics for older adults, compared with the sample. In the United States, as a whole, older adults comprised $16.5 \%$ of the population aged 18 or above, with a mean age of almost 75 years. Older women $(58 \%)$ outnumbered men $(42 \%)$, with $53.3 \%$ of the older adult population married and $31.6 \%$ widowed. Unlike the increasingly diverse general population, more than $85 \%$ of the older adult population was white. Median income for older adults nationally was $\$ 32,158$. Almost $41 \%$ of older adults in the U.S. reported one or more 
Table 2

\section{Characteristics of the Population Aged 65 and Older}

\begin{tabular}{|c|c|c|c|}
\hline & US & State & County \\
\hline$\%$ of Population & 16.5 & 12.8 & 14.6 \\
\hline Mean age in years & 74.8 & 74.2 & 75.1 \\
\hline \multicolumn{4}{|l|}{$\%$ Gender } \\
\hline Male & 42 & 41.4 & 39 \\
\hline Female & 58 & 58.6 & 61 \\
\hline \multicolumn{4}{|l|}{$\%$ Race } \\
\hline White & 85.2 & 93.8 & 86 \\
\hline African American & 8.4 & 5.1 & 12.5 \\
\hline Other & 6.4 & 1.1 & 1.5 \\
\hline \multicolumn{4}{|l|}{$\%$ Marital status } \\
\hline Married & 53.3 & 51.8 & 47.6 \\
\hline Never married & 4.6 & 4.0 & 4.7 \\
\hline Divorced/separated & 10.6 & 9.9 & 11.6 \\
\hline Widowed & 31.6 & 34.3 & 36.2 \\
\hline \multicolumn{4}{|l|}{$\%$ Education } \\
\hline$<$ High school & 27.2 & 40.7 & 29.1 \\
\hline HS/GED & 34.4 & 31.7 & 34.0 \\
\hline Some college & 19.7 & 15.4 & 19.9 \\
\hline College degree or $>$ & 18.7 & 12.2 & 16.9 \\
\hline \multicolumn{4}{|l|}{ \% Disability } \\
\hline With & 40.9 & 48.8 & 43.4 \\
\hline Without & 59.1 & 51.2 & 56.6 \\
\hline \multicolumn{4}{|l|}{$\%$ Poverty } \\
\hline$<100 \%$ & 9.9 & 13.4 & 9.2 \\
\hline $100-149 \%$ & 11.9 & 15.7 & 11.2 \\
\hline $150 \%$ or $>$ & 78.2 & 70.9 & 79.6 \\
\hline $\begin{array}{l}\text { Median income } \\
\text { among adults } \geq 65\end{array}$ & $\$ 25,210$ & $\$ 25,834$ & $\$ 26,633^{*}$ \\
\hline
\end{tabular}


disabilities and almost a quarter lived in poverty, with income under $150 \%$ of the federal poverty level (U.S. Census, 2007).

The older adult population in the largely rural Southern state was somewhat smaller (12.8\%), less diverse (93.8\% white), and with a higher disability level (48.8\%) than in the nation as a whole. Median income was about $\$ 6000$ less than the national median, with $29 \%$ of older adults living at or below $150 \%$ of the poverty level. In the urban area of the state in which the retirement community was located the older population was more diverse ( $14 \%$ non-white) than in the state as a whole. Fewer older adults lived at or below $150 \%$ of the poverty level (20.4\%) and about $5 \%$ fewer older adults lived with disabilities (43.4\%) (U.S. Census, 2007). Despite these facts, the median income $(\$ 26,633)$ was only slightly higher than the state median (Kentucky State Data Center, 2002).

The average age of residents in the target population was estimated by the CCRC to be similar to the national average, i.e., 82 years for independent living and 85 years in assisted living (Harris-Kojetin, Joseph, Zimring, \& Keifer, 2005) with women outnumbering men and an estimated minority population of about 10 to $12 \%$. The CCRC was the only assisted living facility in the state serving low-income older adults. Federally subsidized rent levels for the CCRC were based on U.S. Department of Housing and Urban Development (HUD) income levels: a) Section $8,<\$ 18,000$; b) Section 236, $\$ 18$ to $<\$ 23,250$; and c) Fair Market, $\$ 23,250$ to $\$ 33,000$. HUD considered $\$ 33,250$ or below to be low-income, although in the county where the CCRC was located assistance was available only for incomes under \$23,500 (HUD, 2008b). 


\section{Setting.}

The CCRC was a church-affiliated, non-profit continuing care residential community, open to individuals regardless of religious denomination. The CCRC consisted of two high-rise apartment buildings with a mixture of independent and assisted living apartments in each building, from which the sample was selected. The CCRC also included a rehabilitation unit providing physical, occupational, and speech therapy; a dedicated dementia unit; and a Medicare-certified, state-licensed skilled nursing facility on a single campus. Residents of these units were not included in the study; the stratified random sample was selected only from among residents of independent and assisted living apartments in the two apartment buildings. The CCRC was selected because it was the only identified residential retirement facility in the state that offered assisted living services for low-income older adults. The community employed licensed social workers, certified activity staff, and nursing assistants, while health care services were provided by outside home health agencies and other licensed providers.

\section{Inclusion and exclusion criteria.}

Inclusion criteria for participation in the study were: age 65 or higher, residence in either independent living or assisted living apartments in the CCRC, normal score on the Mini-Cog Dementia Test (Borson, 2000), ability to understand English, and sensory, physical, and cognitive ability to respond to questionnaires, given accommodation for hearing, vision, and literacy deficits. Participants completed the Mini-Cog Dementia Test after signed consent and before administration of the set of questionnaires. All participants, including those with abnormal Mini-Cog scores, were allowed to complete the survey process, unless they became disruptive or distracting, in which case CCRC 
staff were immediately available to discreetly re-locate the participant, according to CCRC policies and procedures. The Mini-Cog Test was a simple screening test for cognitive impairment (memory and executive function) which combined a brief memory test and a clock-drawing test to assess executive function. The Mini-Cog allowed rapid screening for short term memory defects and cognitive abilities that might be impaired in dementia patients. Data from individuals with an abnormal score $(0$ on recall, or 1 - 2 on recall with abnormal clock score) were excluded from the analysis. The Mini-Cog could be administered in as little as 3 minutes and was reported to be as effective in detecting dementia as longer screening tests, with inter-rater reliability of .90 . Sensitivity, specificity, and diagnostic value of the Mini-Cog were comparable to the Mini-Mental State Exam (MMSE) (Folstein, Folstein, \& McHugh, 1975) and Cognitive Abilities Screening Instrument (CASI) (McCurry, et a., 1999), demonstrating higher sensitivity (99\%) and correctly classifies the greatest percentage (96\%) of subjects (Borson, Scanlan, Brush, Vitaliano, \& Dokmak, 2000). Appendix A includes the Mini-Cog Test and instructions for administration and scoring the test.

Sample size.

While power analysis is frequently conducted to determine a priori the number of subjects needed for sufficient power to detect an effect of a desired magnitude, Stevens (2002) pointed out that in multiple regression the focus was on generalizability of the regression equation more than on significance. Sample size must be sufficient for accurate replication, allowing for a certain amount of shrinkage in multiple correlation coefficients $\left(\mathrm{R}^{2}\right)$. While a common rule of thumb for sample size is 5 to 10 participants per predictor, Stevens stated that with 15 participants per predictor, the amount of 
shrinkage would be small $(<.05)$, with a high probability $(.90)$, given a squared population multiple correlation $\left(p^{2}\right)$ of .50 . According to Stevens, in social science research a $p^{2}$ of .50 was considered a reasonable estimate, unless data from the literature supported an alternate estimate of the squared population multiple correlation.

In the present case, an estimated value for $p^{2}$ was available from the feasibility study $(\mathrm{N}=19)$ conducted in preparation for the dissertation study (McCarthy, 2009). The feasibility study found a significant bivariate correlation (.53) between self-transcendence and successful aging. Squaring the correlation coefficient resulted in a $p^{2}=.28$. Given the small sample size of the feasibility study and its limited power, it appeared reasonable to assume the $p^{2}$ of the dissertation study would more closely approach .50 . Thus sample size was chosen to be mid-way between a $p^{2}$ of .25 and .50 . Extrapolating from a table provided by Stevens (2002, p. 146-7), for six predictor variables, an error rate of .05 error and $95 \%$ probability (increased from .90 ), a conservative approach was taken and a desired sample size of 135 was selected for the study to maximize the potential for finding significance and minimizing Type I error. Based on the feasibility study it was estimated $10 \%$ of participants who completed surveys would subsequently be excluded due to abnormal dementia screening. Thus the study would over-sample if necessary, enrolling approximately 150 participants to obtain data on 135 non-demented participants, with selection continuing until 135 non-demented participants completed surveys.

\section{Recruitment.}

To facilitate recruitment, the investigator was introduced at regular monthly resident meetings in each of the two high-rise buildings, and briefly described the study, 
congruent with information on the recruitment flyer, displayed in Appendix B. At both meetings, residents were told that names, telephone numbers, apartment numbers and rent levels would be provided so that the investigator could contact a random sample by telephone. Residents were told if they preferred not to be telephoned they should tell the investigator or the Director of Social Services. The study was also featured in an item in the monthly resident newsletter. Additionally, recruitment flyers were placed in mailboxes (not postal boxes) at each resident's apartment door and at key locations throughout the two apartment buildings.

Residents were told the research study would explore how older adults see life and adjust to aging and that they would be asked to answer questions about growing older. Residents were also told that while the results of the study might be used in future to help plan health and social policies or programs and to provide aging services based on the information provided by the participants, immediate benefits to participants would be limited to satisfaction with knowing their knowledge and experience might be of help to others. Other information presented in residents' meetings, the newsletter, or the flyer included that telephone calls would be made to residents chosen at random, thus not all residents would be contacted; that participation in the study was voluntary; and that participants would be compensated for their time with a $\$ 5$ gift card to a market located in the CCRC. Residents who agreed to participate were asked to meet in a private meeting room at the CCRC, for about 45 minutes to 1 hour, with the investigator and a small group of other residents (5 to 7), to complete a set of questionnaires. Residents were told the questionnaires would be in large print and would be read aloud by the investigator. Residents were also told that they would be asked to complete a brief 
assessment (Mini-Cog Test) to document their ability to understand and answer the survey questions.

\section{Selection.}

A sampling frame was provided by the CCRC. The sampling frame included the names of all residents in both high-rise buildings, unless the resident opted out. Other information provided was: apartment number, telephone number, and rent level. Status as either independent or assisted living was indicated by apartment numbers. The sampling frame was divided into two groups, residents in independent living apartments and residents in assisted living apartments. The sampling frame was then randomized, using a computerized randomization program. The number of participants from independent or assisted living groups was proportionate to total numbers of residents in each group in the target population. Potential participants were contacted by telephone and asked to participate in the study. Based on the feasibility study (McCarthy, 2009), a response rate of approximately $33 \%$ was considered achievable for a survey study of older adults in a residential setting. Data collection sessions were scheduled daily, Monday through Friday at the following times: $10 \mathrm{am}, 2 \mathrm{pm}, 4 \mathrm{pm}, 6 \mathrm{pm}$, with appointments recorded on the Schedule of Data Collection Sessions shown in Appendix C. Participants were offered a choice of sessions.

Residents who agreed to participate received written reminders, in sealed envelopes, with date, time and location of the data collection session, and the name and phone number of the investigator. The reminders were placed in non-postal mailboxes outside apartment doors the day before the scheduled session. It was expected that a large number of residents with dementia, due to memory loss and loss of executive function, 
might not attend data collection sessions as scheduled, despite the appointment reminder cards, minimizing the number of participants subsequently excluded due to abnormal dementia tests. An example of the appointment reminder is shown in Appendix D. Copies of the Consent (Appendix E) and Health Insurance Portability and Accountability Act (HIPAA) Authorizations (Appendix F) were included in the sealed envelope to allow participants ample time to consider the documents in advance, but were not signed until the data collection session. Participants retained copies for their records. A written schedule of participant names and the dates and times of data collection sessions was maintained, accessible only to the investigator. An example of the written schedule of data collection sessions is shown in Appendix C.

\section{Measures}

Three major constructs were operationalized for the study, transcendence, adaptation, and successful aging. Measurement instruments for use in the present study were selected, following review of theoretical and empirical literature, for congruence with the conceptual definitions proposed in the Theory of Successful Aging (Flood, 2005), and for demonstrated reliability and validity among community-dwelling older adult, English-speaking populations. Results of a feasibility study (McCarthy, 2009) also contributed to decisions on selection of instruments. Permissions were obtained from the authors for the use of each instrument. An investigator-developed questionnaire was used to obtain sociodemographic data, responses to questions on health and functional status, and to a single dichotomous question on perceived successful aging and data was obtained from the sampling frame provided by the CCRC. Table 1 on page 17 displayed the operationalized definitions and measures selected for all study variables. 


\section{Adaptation.}

Adaptation was operationalized using the 14-item Proactive Coping subscale of the Proactive Coping Inventory (PCI) (Greenglass, 2002). See Appendix G for a copy of the instrument and instructions for administration and scoring. While Roy's (1984) adaptation model was used as the theoretical basis for adaptation in Flood's (2005) successful aging theory, no validated instrument based on Roy's Adaptation Model was identified in the literature for use among older adults. Also, as discussed in the literature review, Roy's model was not congruent with life span development theory or with current nursing views on individuals' autonomy and rights to decision-making. The three coping mechanisms drawn from Roy's theory which resulted in adaptation, i.e., functional coping, intrapsychic coping, and spiritual coping were largely congruent with components of proactive coping, as described by Schwarzer and Taubert (2002).

The 55-item Proactive Coping Inventory (PCI) based on Schwarzer and Taubert's theory (2002) was composed of 7 subscales, designed to be used singly or together. The PCI (Greenglass, et al., 1999) was developed among a sample of English-speaking Canadian university students $(\mathrm{N}=252$; mean age 21.7) and refined among a sample of Polish-Canadian university students and adults $(\mathrm{N}=144 ; 18$ students \& 126 adults, mean age 38.93) with internal consistency demonstrated by reliability measures $(\alpha)$ of .85 and .80 in the two samples. Convergent and discriminant validity were demonstrated by statistically significant positive correlations with the Proactive Attitude Scale (Schwarzer, 1999) ( $\alpha=.76)$; the General Perceived Self-Efficacy Scale (Schwarzer, 1998) $(\alpha=.78)$; 
the Brief COPE scales (Carver, 1997) $(\alpha=.50$ to .90); and the Hopkins Symptom Checklist (Derogatis, Lipman, Rickels, Uhlenhuth, \& Covi, 1974) $(\alpha=.86)$.

The proactive coping subscale of the PCI used in the study measured cognitive goal recognition, resource accumulation, and goal-seeking behavior, with a proactive focus on personal growth, well-being, and finding meaning in life. Higher scores on the Proactive coping subscale were associated with a positive outlook and a disposition toward both assimilative and accommodative coping (Greenglass, et al., 1999). In a study that examined how well life satisfaction of young, middle-aged, and older adults was predicted by locus of control, proactive coping ability, and self-efficacy $(\mathrm{N}=240)$, comparing young, middle-aged, and older adults, the PCI found younger adults were most proactive in coping with stress, and older adults were least proactive, although older adults were most satisfied with life (Simons, 2002). A study among 224 communitydwelling older adults attending a community center (mean age 75) used the Proactive Coping subscale of the PCI to examine proactive coping, social support, daily hassles, functional disability, and living arrangements (Fiksenbaum, Greenglass, \& Eaton, 2006). Proactive coping was significantly negatively related to hassles and functional disability. Proactive coping and living arrangements were positively related to social support. Test/re-test stability was $.80-.85$.

The feasibility study (McCarthy, 2009), as previously reported, found statistical significance for a model regressing successful aging (SAI) on proactive coping, operationalized using the proactive coping subscale, and self-transcendence $(F=4.592$, $p=.027$ ), which explained $36.5 \%$ of the variance. While the bivariate correlation of SAI with PCI $(r=+.340)$ was not statistically significant, the correlation was larger than other 
instruments for adaptation and it was expected would be significant with a larger sample and greater power. Moderate reliability of the proactive coping subscale was demonstrated in the feasibility study by a Cronbach's alpha .62 . The instrument was also brief and well-received by participants, thus the Proactive Coping Subscale of the PCI was selected to operationalize adaptation in the study. Ultimately, the PCI yielded a Cronbach's alpha of .77 in the present study. A copy of the Proactive Coping Subscale of the PCI with instructions for administration and scoring is found in Appendix G.

\section{Transcendence.}

Transcendence was operationalized using Reed's (2003) Self-transcendence Scale (STS), displayed in Appendix H. Because Tornstam's $(1989,1996)$ theory of gerotranscendence provided the theoretical basis for the construct in Flood's successful aging theory (2005), the Gerotranscendence Scale (GTS) was considered to operationalize transcendence for the study, but was not selected. A brief discussion of the GTS follows to support the decision to use an alternate instrument.

Tornstam's (1994) 10-item Gerotranscendence Scale (GTS) was originally developed among a sample of adults in 1995 (aged 20 - 85) and refined in 2001 (aged 65 and older) (Tornstam, 2003, 2005). The GTS was largely used among Scandinavian samples. There was no validated English language version of the GTS and it has not been validated among an English-speaking population. In instrument development (Tornstam, 2003), alpha statistics for three dimensions on the scale were: cosmic .73 , coherence .57 , and solitude .60 . Only cosmic transcendence was statistically significant and overall alpha for the total scale was not reported. Convergent validity was reported for the GTS based on a statistically significant $(p<.001)$ correlation with a single-item question on life 
satisfaction $($ cosmic, eta $=.16$; coherence, eta $=.50$; solitude, .16$)$. No divergent validity was reported (Tornstam, 2003). These findings appeared to support criticisms on the validity and reliability of the GST, specifically, that empirical findings were not consistent with the theory of gerotranscendence (Jönson \& Magnusson, 2001).

In a recent feasibility study (McCarthy, 2009), internal reliability of the GTS (Tornstam, 2003, 2005) was poor (Cronbach's alpha .58). The bivariate correlation of the GTS with SAI, was not only non-significant $(-.021, p=.933)$ but the direction was negative. A model regressing SAI on GTS and measures of adaptation was not statistically significant $(F=1.566, p=.240)$. Perhaps due to cultural and language differences, several items on the GTS were abstract and confusing to some participants in the feasibility study, such as, "feeling connected with the entire universe," "feeling the presence of people who are elsewhere," and "living in the past and present simultaneously."

The unidimensional STS scale selected to operationalize gerotranscendence consisted of 15 items on a four-point Likert-type scale which measured older adults' perceptions of the degree or level of transcendence, ranging from 1 for "not at all" to 4 for "very much." The STS used summative scoring and was designed to be administered as an interview or a questionnaire. Items included: "sharing my wisdom and experience with others"; "helping others in some way"; and "finding meaning in my past experiences." Reported internal validity was demonstrated by Cronbach's alphas ranging from .80 to .88 (Coward, 1990). Test/re-test stability was also reported (.70 to .95 ) (Decker \& Reed, 2005; Scherwitz, Pullman, McHenry, Gao, \& Ostaseski, 2006). 
According to Reed (electronic communication, January 11, 2008), content validity was based on a thorough literature review of empirical and theoretical literature to specify the domain of content, with careful construction and refinement of items. Support for construct validity was found in the relationship of STS scores with other measures and in groups which scored on the STS as theorized, in a phenomenological study of selftranscendence, and in secondary analysis of data from correlational and longitudinal studies on developmental resources (e.g. Coward, 1990; Reed, 1991).

In the feasibility study reported above (McCarthy, 2009), STS was positively and significantly correlated with successful aging (Pearson $r=.533, \mathrm{p}=.02$ ). Internal validity was demonstrated for the STS (Cronbach's alpha .72). A model regressing the STS and proactive coping on SAI was statistically significant $(F=4.592, p=.027)$, explaining $36.5 \%$ of the variance, despite the small sample size $(\mathrm{N}=20)$. The standardized regression coefficient for STS was also significant $(\beta=.502, t=2.504, p=.023)$, independent of adaptation.

For these reasons Reed's $(1986,1989 b)$ the Self-transcendence Scale was selected to operationalize the construct. As discussed in the review of the literature, Selftranscendence theory (Reed, 2003) was largely congruent with gerotranscendence (Tornstam, 2003, 2005), was developed for an English-speaking late life population, and was well-validated among older adults (Reed, 1989b). In instrument development, the factor of self-transcendence accounted for $80 \%$ of the variance (Reed, 1986), while in the feasibility study (McCarthy, 2009) the STS was significantly correlated with the SAI and contributed to a statistically significant regression model. Thus the Self-transcendence Scale was selected to operationalize transcendence for the study. The Cronbach's alpha 
found for the STS in the present study was .73. See Appendix H for a copy of the Selftranscendence Scale with instructions for administration and scoring.

\section{Successful aging.}

A newly developed 20-item survey instrument to measure successful aging, the Successful Aging Inventory (SAI) (Flood, 2008), was used to operationalize the outcome variable in the present study. Appendix I provides a copy of the instrument. Self-report scores on the Likert-type scale ranged from 0 for "hardly ever" to 4 for "almost always" in response to a statement asking "how you feel right now." Sample items included, "I manage to do the things I need to do to take care of my home and to take care of myself (eating, bathing, dressing);" "I would rather have a few close friends than many casual ones;" and "I feel able to cope with life events."

The SAI (Flood, 2008) was developed using a convenience sample of communitydwelling older adults $(\mathrm{N}=200)$ attending a senior citizens center, with a Cronbach's alpha of .88. Statistically significant correlations of the SAI demonstrated convergent validity: .378 with the Life Satisfaction Inventory-Aging scale (LSI-A) (Neugarten, Havighurst, \& Tobin, 1961); .544 with the Purpose in Life Test (PIL) (Crumbaugh, 1968; Crumbaugh \& Maholick, 1964); and .406 with a measure of perceived control, the Mastery Scale (Pearlin, et al., 1981). The SAI was negatively correlated (-.317) with the CES-D scale for depression (Radloff, 1977), demonstrating divergent validity. Principal Components Analysis identified two factors which together accounted for $47.78 \%$ of the variance: coping/adapting (36.4\%), and existential (11.38\%). Test/re-test statistics have not yet been reported. 
In the feasibility study (McCarthy, 2009), the SAI demonstrated good internal reliability (Cronbach's alpha .72). Considering the small sample size for the feasibility study, this alpha was considered reasonable, in comparison to the Cronbach's alpha obtained in instrument development (.88). Subsequent to data analysis for the present study, the Cronbach's alpha was .82. The SAI instrument, with instructions for administration and scoring, is found in Appendix I.

\section{Investigator-developed questionnaire.}

Other study variables were operationalized using participant responses to an investigator-developed questionnaire for sociodemographic information, including five control variables: age, income level, functional status, perceived health, and number of chronic diseases. The investigator-developed questionnaire is shown in Appendix J. Sociodemographic data obtained included age (in years), gender (male/female), race (African American/White/Other); marital status (married or partnered/never married/divorced or separated/widowed); and education level (less than high school diploma/high school graduate/some college/ college graduate or higher). The investigator-developed questionnaire also was used to obtain data on perceived income, health, and functional status. Self-reported income was operationalized for older adults as one of three classifications used by Flood, $(2006,2008)$ (barely enough to get by/ enough to get by/ enough to live in some luxury). A summative index constructed from perceived health (very good/good/fair/poor) and number of chronic diseases (hypertension or high blood pressure/ arthritis/ heart disease/ diabetes/ lasting effects of a stroke/ respiratory disease such chronic bronchitis, asthma, or emphysema/ cancer/other) (U.S. Centers for Disease Control and Prevention (CDC), (CDC, 2008, n.d.) was considered to 
operationalize health but ultimately perceived health and number of chronic diseases were entered in the analysis as separate variables. Self-reported functional status was operationalized as independent (able to live independently, without help from another person more than occasionally) or assisted (needing assisted living services such as help with bathing, medications, or getting around).

The final study variable on the investigator-developed questionnaire was a dichotomous response (yes/no) to the question, "Do you consider yourself to be successfully aging?" In the recent feasibility study (N=20) (McCarthy, 2009) all responses to this item were positive, thus there was no variability and statistical analysis was not conducted. As it was possible that greater variability might be found with a larger and more diverse sample, and as the question imposed low participant burden, the questions was retained for the dissertation study. However, it was anticipated that responses to the question might remain overwhelmingly positive, which proved to be the case.

\section{Sampling frame.}

In the feasibility study (McCarthy, 2009), it was observed that a large number of non-demented participants stated they could not accurately recall their current annual income because family members or other representatives managed their financial affairs. Thus, rather than rely on self-reported income, income level was defined for the study by rent levels provided by the CCRC on the sampling frame. Rent levels were based on U.S. Department of Housing and Urban Development (HUD) state and county criteria for lowincome housing: Section $8(<\$ 18,000)$, Section $236(\$ 18,000$ to $<\$ 23,250)$, or Fair market (\$23,250 to $\$ 33,000)$ (HUD, 2008a,b). All three rent levels were classified as low-income and were below $300 \%$ of the federal poverty guidelines (U.S. Department of Health and Human Services (DHHS), 2009). 
Functional status, indicated by assignment to either an independent living or assisted living apartment, was based on criteria established by the CCRC. CCRC criteria for independent living included residence in an apartment (studio or one-bedroom) with a door that may be locked, private bath and kitchen, while receiving services limited to assistance with instrumental activities of daily living (IADLs) such as shopping, meals, housekeeping, and laundry. Assisted living, as designated by the CCRC, included all independent living services, with the addition of personal care, including one or more of the following: a) more than incidental assistance with activities of daily living (ADLs) such as bathing and hygiene, dressing, transfer among bed, chair or wheelchair, ambulation, feeding, or toileting; b) supervision of self-administration of medications; and/or c) reminder or escort service to meals, activities, or other locations within the retirement community.

\section{Data Collection}

Data collection was conducted in private meeting rooms in the apartment building in which the participants resided. CCRC staff was immediately available to assist with relocating any scheduled participant who was disruptive or distracting, to allow the investigator to remain focused on the group as a whole. Unless a resident was disruptive or distracting, participants were allowed to complete the survey questionnaires regardless of the Mini-Cog score. However, responses for participants with abnormal Mini-Cog scores were not used in data analysis or counted toward the total number of participants. Based on the feasibility study (McCarthy, 2009), it was estimated that approximately $10 \%$ of participants would test abnormally for dementia, thus it was anticipated that 
approximately 150 participants would be needed to obtain a non-demented sample size of 135.

All data collection sessions were conducted by the investigator, during a single session, with no additional reminders or follow-up. A standardized protocol was followed for each data collection session, as displayed in Appendix K. All surveys were in 14point font, large-print examples of Likert scales were provided, and all items were read aloud at least twice to accommodate sensory or literacy deficits. See Appendix L for examples of the enlarged Likert scales.

After the study aims and procedures were explained by the investigator and prior to administration of the Mini-Cog (Borson, 2000) dementia screening test, written informed consent and authorization for collection of health information were obtained. The Consent document is shown in Appendix E and the HIPAA Authorization in Appendix F. Copies of the Consent and HIPAA documents were provided along with appointment reminders, prior to the data collection session to allow the participant to examine the forms in advance. These copies remained with the participants for their records; additional copies were also available at data collection sessions. Standardized instructions included admonitions to refrain from talking or suggesting answers to others during data collection, an explanation for why the investigator could not explain or rephrase items, and a request to refrain from discussing the survey with other CCRC residents after the session was completed.

The Mini-Cog Test (Borson, 2000) was administered according to instructions provided in Appendix A. The Mini-Cog was explained with the statement, "Now, we will begin with a little memory test. Don't worry about 'passing' or 'failing.' It's just to have 
a record, showing people who completed the surveys were able to understand instructions and answer questions." If distracting or disruptive behaviors associated with dementia occurred, the resident would be discreetly re-located by retirement community staff, according to CCRC policies and procedures. In the absence of disruptive or distracting behavior, all participants were allowed to complete the questionnaires regardless of MiniCog score. However, responses for participants with abnormal Mini-Cog scores were not entered in data analysis, nor were they counted toward the number of participants. As specified in the HIPAA Authorization signed by participants, the Director of Social Services was notified of abnormal results of Mini-Cog tests, so that appropriate actions could be taken, according to CCRC policies, to ensure participants' safety and welfare.

Survey questionnaire packets included Consent and HIPAA documents, the MiniCog test form, a set of laminated cards with examples of the Likert scale used for each instrument, copies of each measurement instrument, and a receipt for a $\$ 5$ gift card, shown in Appendix M. To accommodate problems with communication, sensory deficits or limited literacy. the investigator spoke slowly, distinctly, and in a low pitch, and loudly enough to be heard by respondents, even those with some hearing loss. Participants' attention was directed to enlarged versions of each scale and to instruments in 14-point font, which were explained prior to beginning each survey instrument. Each item was read aloud at least twice. If a participant did not understand the question, the item was reread, making an effort to communicate clearly. Items were not explained or re-phrased. The only admissible clarification was an exact repetition of the item as it appeared on the scale or to direct a participant to the enlarged response scale. Participants marked their own responses on the survey questionnaires. At the conclusion of data collection, 
participants were thanked and reminded not to discuss the study with other CCRC residents. Five dollar gift cards to a small market located in the CCRC complex were provided as compensation for participants' time. The location of the market accommodated limited mobility and transportation among some participants. Upon receipt of the \$5 gift card participants signed individual receipts, included as the last page of the survey questionnaire packets. An example of the receipt is shown in Appendix J. When all survey questionnaires were completed, participants returned the packet of survey questionnaires to the investigator, and then a brief discussion was held to allow participants to ask questions or make comments, while participants' impressions of the survey process were elicited by the investigator.

\section{Data Management}

When each data collection session was completed and participants had cleared the room, the investigator processed the packets to maintain confidentiality, according to the standardized protocol. First the Mini-Cog was scored and the result entered in the space provided on the investigator-developed questionnaire. Depending on the Mini-Cog score a unique identifier was assigned each participant. Surveys with normal scores (ID 01-1, 01-2, 01-3 ...) were included in data analysis. Surveys with abnormal scores (ID 02-1, 02-2. 02-3 ... ) were excluded. Next the participant's name and the respective identifier was recorded on a study roster, displayed in Appendix N. Then identifying documents (Consent, HIPAA, and gift card receipt) were removed and stored separately from the survey packets. The investigator maintained possession of both the identifying documents and the surveys until they were transported to the university school of nursing where surveys and identifying documents were maintained separately in locked cabinets in a 
secure area of the school with limited access. Data was entered in a secure database maintained by the school of nursing. Only aggregated results were shared as specified in the Consent and HIPAA documents.

Data Analysis

Data was entered in Version 13.0 of the Statistical Program for Social Science (SPSS) software. Descriptive statistics including frequencies, means, standard errors and ranges were obtained. Pearson correlation coefficients, Spearman's rho, and one-way ANOVA were used to explore relationships among the variables. Multiple regression was used to examine the influence of the predictors, transcendence and adaptation, on the single outcome variable, that is, successful aging, controlling for age, income, health, and functional status. Binary logistic regression was intended to determine if differences in scores on the SAI existed between those subjects who reported they were aging successfully and those who did not, if there was sufficient variability in the responses. Appropriate tables or figures were employed to illustrate the characteristics of the sample compared to population means derived from the literature and relationships among the variables.

\section{Ethical Considerations}

A written Letter of Agreement was provided by the CCRC specifying approval for data collection at the site, and support for the study including provision of the sampling frame, access to resident non-postal mailboxes, adequate space for data collection sessions, and staff availability to manage disruptive or distracting residents with dementia. A copy of the letter is shown in Appendix O. 
Expedited approval was requested from the University of Louisville ( $\mathrm{U}$ of $\mathrm{L}$ ) Institutional Review Board (IRB). Subsequently, based on minimal risk of harm associated with a survey study which did not tap sensitive information, among a population of non-cognitively impaired older adults exempt status was obtained. Letters of approval from the IRB are included in appendix P. Provisions were made with retirement community administrative and/or social service staff to provide assistance and emotional support should a respondent become distressed in response to participating in the study, if a participant received an abnormal Mini-Cog (Borson, 2000) test score, or if dementia-type behavior was observed.

Written authorization for use of protected health information was obtained, as required by the Health Insurance Portability and Accountability Act (HIPAA) for identifiable protected health information. Although no medical records were accessed, HIPAA provisions for protected health information considered collection of age in years for respondent over the age of 89 to be identifiable; responses to questions on perceived health and number of chronic disease conditions constituted protected health information. The HIPAA authorization is shown in Appendix F.

Informed, written consent was obtained prior to administration of the Mini-Cog (Borson, 2000) screen or survey questionnaires. Confidentiality was preserved, using secure storage of paper documents and computerized data. Access to the data, other than in aggregated form, was limited to researchers or authorities approved by the University of Louisville Institutional Review Board, except if abuse or neglect of an older adult, reportable by law, was observed or reported to the investigator, or if immediate danger to 
self or others was observed, in the judgment of the investigator, a licensed registered nurse.

Participants were selected at random from a sampling frame of all residents of independent or assisted living apartments in the CCRC, unless a resident requested to opt out of the sampling frame. Both women and men were included in the study; women constitute a majority of residents in the retirement community and in the aging population as a whole, and also the majority of participants in the study. Children were not included in the study, due to inclusion criteria based on age 65 or older. Approximately 10 to $12 \%$ of residents of the CCRC were members of a minority population. As racially diverse a sample as possible was sought, within the limits of the available target population and the process of random selection. Ultimately, African Americans participated in the study in greater proportion than African Americans were represented in the target population.

\section{Limitations}

Limitations of the study included the cross-sectional study design, which provided information on only one point in time. The potential for both self selection bias and social desirability bias were also limitations. Despite random selection, it was likely that older adults who were satisfied with their aging would be more likely to consent to participate in the study. Participants who did consent might also be more likely to state they were aging successfully, as it was a socially desirable response. Additionally, the narrowly defined target population limited the generalizability of study findings. Another limitation involved the newly developed instrument selected to measure successful aging, the Successful Aging Inventory (SAI) (Flood, 2008), which had not been previously 
validated. Finally, the desired sample size of 135 was not achieved, which will be discussed in chapters four and five.

\section{Summary}

The dissertation study is an exploratory study conducted to explore the potential usefulness of a new mid-range nursing theory, (Flood, 2005) which merged competing biomedical and psychosocial perspectives, and added important factors identified in a limited numbers of studies looking at older adults' own criteria for success, including social and environmental factors and an existential or spiritual perspective. The descriptive cross-sectional, correlational study was designed to examine the size and direction of relationships among two independent variables, transcendence and adaptation, and a single dependent variable, successful aging (Flood, 2005). The theory hypothesized that together transcendence and adaptation contribute to successful aging, viewed from the individual older adult's perspective. A stratified random sample of nondemented older adults, aged 65 and older, living independently or receiving assisted living services, was drawn from a sampling frame consisting of about 390 residents in a low-income continuing care retirement community (CCRC) operated by a charitable, non-profit organization in an urban county of a Southern state.

Flood's Theory of Successful Aging addressed concerns about the limited potential for successful aging among older adults with chronic disease and disability, low socioeconomic status, or subject to bias based on age, gender, or race. Because of the increasing numbers of older adults, and the potential demands that will be placed on societal resources, it is important to identify factors that support successful aging, both to meet the national goal of increasing not only years of life, but quality of life in those 
years (Healthy People 2010, 2000; The United Nations (U.N.), 2000; World Health Organization (WHO), 1999), and the goal of eliminating health disparities (National Institute of Health (NIH), 2008). 


\section{CHAPTER IV}

\section{RESULTS}

This chapter presents results of the dissertation study. Procedures for data preparation are described, as well as statistical power and assumptions on statistical tests. Descriptive statistics are presented for variables as well as results of statistical tests addressing research questions. Statistical analyses used to answer four research questions include tests of significance for correlations and for tests of differences in group means based on sociodemographic characteristics, age, income, function, and health. Multiple regression is used to explore relationships among the outcome variable, SAI, with two predictors, PCI and STS. Binary logistic regression was not conducted due to lack of variability in responses to a dichotomous question on perceived successful aging.

\section{Data Preparation}

One hundred and twenty-three residents were guided through the informed consent process, $80 \%$ in independent living and $20 \%$ receiving assisted living services. The percentages were proportionate to the CCRC's census. Ten $(8 \%)$ cases were subsequently excluded due to age $(2<65$ years old $)$ or abnormal scores on the Mini-Cog screen for dementia (8 cases), fewer than the projected $10 \%$ exclusion level. Data obtained on the surveys were entered into Version 13.0 of the SPSS computer program. 
One case was eliminated from analysis due to multiple missing values (>10\%). Less than $5 \%$ missing values were found for all other cases, thus the final sample size was 112 . Mean scores were substituted for missing values in the two summative scales, the Proactive Coping Inventory (PCI) (Greenglass, 1999, 2002) and the Successful Aging Inventory (SAI) (Flood, 2008), but not on the Self-transcendence Scale (STS) (Reed, 1989b) because scoring instructions for the STS included calculating a mean based on number of actual responses, accommodating for missing values.

Data were screened to identify potential outliers or influential datapoints, and to check for normality. Four potential outliers on the variables were identified, on the STS (2) and on the SAI (2); none were influential, meaning the distance from the mean was less than three standard deviations, when examined using casewise diagnostics. To preserve potentially important information from participants with extreme scores, influential data points were retained in the data set. To reduce skewness and kurtosis, transformations (square root, natural logarithmic, log10, and inverse) were attempted but failed to improve the fit by more than 1 to $2 \%$ (Norusis, 2006). Table 3 displays descriptive statistics for the outcome variable (SAI), two predictors (PCI and STS), and five control variables (age, income, function, perceived health, and number of chronic diseases).

Other data preparation involved reverse coding items on two scales per scoring instructions (three items on the PCI and one item on the STS). A categorical ordinal level variable for age was created, with definitions defined by Garfein and Herzog (1995): 
Table 3

Descriptive Statistics for Predictor, Outcome, and Control Variables $(n=112)$

\begin{tabular}{lcccccccc}
\hline & $\begin{array}{c}\text { Proactive } \\
\text { Coping }\end{array}$ & $\begin{array}{c}\text { Self- } \\
\text { Transcendence }\end{array}$ & $\begin{array}{c}\text { Successful } \\
\text { Aging }\end{array}$ & $\begin{array}{c}\text { Age in } \\
\text { Years }\end{array}$ & $\begin{array}{c}\text { Perceived } \\
\text { Health }\end{array}$ & $\begin{array}{c}\text { Chronic } \\
\text { Disease }\end{array}$ & $\begin{array}{c}\text { CCRC } \\
\text { Rent }\end{array}$ & $\begin{array}{l}\text { CCRC } \\
\text { Function }\end{array}$ \\
Mean & 41.09 & 3.45 & 62.53 & 79.85 & 2.46 & 2.85 & 1.53 & 1.21 \\
Median & 42.00 & 3.47 & 64.00 & 80.00 & 2.00 & 3.00 & 1.00 & 1.00 \\
SD & 6.87 & .347 & 9.32 & 7.76 & .770 & 1.30 & .838 & .406 \\
Skewness & -.216 & -1.123 & -.735 & -.038 & -.029 & .060 & 1.085 & 1.479 \\
Std. Error & .228 & .228 & .228 & .228 & .228 & .228 & .228 & .228 \\
of Skewness & & & & & & & & \\
\\
Kurtosis
\end{tabular}


young-old (age 60-69), old-old (age 70-79), and oldest-old (age 80 and above). A second categorical ordinal level variable was created for perceived health, with definitions as follows: very good, good, fair, and poor. Finally, a dichotomous variable for rent payment was created, with rent defined as subsidized (Section 8 and Section 236) or unsubsidized (Fair Market Rent level). Function remained a dichotomous variable as originally defined, i.e. independent or assisted.

Power

The health index originally considered as a composite score for health will be discussed further, but was not included in the analysis. Therefore the number of control variables increased from four to five, increasing total independent variables from six to seven. Steven's (2000) criteria of at least 15 cases per variable for a squared population multiple correlation $\left(p^{2}\right)$ of .50 with .90 probability of a decrease in $p^{2}$ of less than .10 was used to determine the desired sample size $(\mathrm{N}=135)$. The decision on desired sample size took into account a $p^{2}$ of .25 in a prior feasibility study $(\mathrm{N}=20)($ McCarthy, 2009) and increased probability to .95 . While the number of participants completing surveys $(\mathrm{N}=123)$ was smaller than desired, the final sample size $(\mathrm{n}=112)$, with seven variables, was estimated to meet Steven's criteria for $95 \%$ probability of a decrease in $p^{2}$ of less than .10 at a $p^{2}$ of .50 . The strength of the evidence based on a single feasibility study that $p^{2}$ was only .25 , rather than .50 , which is generally assumed in social sciences, was considered and the decision was made to proceed with data analysis, although it was recognized the potential for finding significance was somewhat diminished.

Multiple regression may be used for two purposes: (a) explanatory, to identify relationships among a set of predictors and an outcome variable; or (b) predictive, to 
develop a model that can be replicated when applied to future studies. In the present case, the primary purpose for the regression analysis was to determine if, and to what degree, adaptation and transcendence predict successful aging. Statistical significance was found for a regression model, with sufficient effect size to indicate the potential for not only statistical significance, but also practical or clinical significance. Sample size may have limited power for finding significance for correlations and tests of differences in the mean and interpretation of the findings reflects this limitation.

Beyond finding significance for a regression analysis, a second concern is the predictive power of the model when applied to future studies; a sufficiently large coefficient of determination $\left(\mathrm{R}^{2}\right)$ would be required to allow for shrinkage for a predictive model to replicate accurately (Steven, 2000). In regression, the value of $R^{2}$ might be inflated, resulting in a statistically significant model that does not replicate when applied to other samples from the same population. Two factors might inflate the $R^{2}$. First, the addition of each variable to the model, regardless of its statistical significance, would inflate the $R^{2}$ value. A second factor that would inflate the $R^{2}$ is the fact that all models fit the sample better than the population from which the sample is drawn, and this is especially true for smaller sample sizes (Noruŝis, 2006). The adjusted $R^{2}$ is a statistic that corrects for both of these factors (Noruŝis, 2006). Thus the adjusted $R^{2}$ S was interpreted for this study.

\section{Statistical Tests and Assumptions}

To answer the research questions, multiple regression analysis was the primary statistical technique used (Stevens, 2000). Multiple regression is robust to violations of assumptions such as normality and influential data points and accommodates continuous 
and dichotomous data, as well as categorical data with more than two factors when dummy coded. Summary or mean scores on the PCI, STS, and SAI provided continuous level data. The PCI was normally distributed. The STS exhibited both negative skewness and kurtosis, but did not significantly depart from normality (Shapiro-Wilks test, $p=$ .187). The SAI had acceptable skewness but demonstrated significant kurtosis, largely due to the effects of a single extremely low data point. Based on inspection of diagnostic plots, the investigator concluded that the linearity, normality, and homoscedasticity assumptions of multiple regression were met.

Spearman's rho, a non-parametric test, was used to examine categorical and nonnormally distributed variables (Munro, 2005). For normally distributed variables parametric tests used included Pearson's correlation coefficients and independent t-tests for continuous variables, and one-way analysis of variance for continuous variables grouped by categorical variables with two or more levels. Finally, binary logistic regression (Noruŝis, 2006) was planned to identify differences in values on the PCI, STS, and SAI related to participants' responses to a dichotomous question on perceived successful aging.

Description of the Sample

Participants' ages ranged from 65 to 95 years, with a mean of almost 80 years; the mean was in line with national averages for residents of independent and assisted living facilities (Harris-Kojetin, Joseph, Zimring, \& Keifer, 2005). Table 4 displays characteristics of the sample, compared with national, state, and local populations. Women constituted more than $80 \%$ of subjects. Similarly, $49 \%$ of participants were widowed. Participation of African Americans in the study, at 23.2\%, exceeded national 
Table 4

Characteristics of the Sample Compared to the Population Aged 65 or Older

\begin{tabular}{|c|c|c|c|c|c|}
\hline & US & State & County & & Sample \\
\hline$\%$ of Population & 16.5 & 12.8 & 14.6 & & \\
\hline Mean age in years & 74.8 & 74.2 & 75.1 & Ran & $\begin{array}{l}79.9 \\
65-95\end{array}$ \\
\hline \multicolumn{6}{|l|}{$\%$ Gender } \\
\hline Male & 42 & 41.4 & 39 & & 19.6 \\
\hline Female & 58 & 58.6 & 61 & & 80.4 \\
\hline \multicolumn{6}{|l|}{$\%$ Race } \\
\hline White & 85.2 & 93.8 & 86 & & 76.8 \\
\hline African American & 8.4 & 5.1 & 12.5 & & 23.2 \\
\hline Other & 6.4 & 1.1 & 1.5 & & 0 \\
\hline \multicolumn{6}{|l|}{ \% Marital status } \\
\hline Married & 53.3 & 51.8 & 47.6 & & 10.7 \\
\hline Never married & 4.6 & 4.0 & 4.7 & & 12.5 \\
\hline Divorced/separated & 10.6 & 9.9 & 11.6 & & 22.7 \\
\hline Widowed & 31.6 & 34.3 & 36.2 & & 49.1 \\
\hline \multicolumn{6}{|l|}{$\%$ Education } \\
\hline$<$ High school & 27.2 & 40.7 & 29.1 & & 23.2 \\
\hline HS/GED & 34.4 & 31.7 & 34.0 & & 33.9 \\
\hline Some college & 19.7 & 15.4 & 19.9 & & 26.8 \\
\hline College degree or $>$ & 18.7 & 12.2 & 16.9 & & 16.1 \\
\hline \multicolumn{6}{|l|}{ \% Disability } \\
\hline With & 40.9 & 48.8 & 43.4 & Assisted & 20.5 \\
\hline Without & 59.1 & 51.2 & 56.6 & Independent & 79.5 \\
\hline \multicolumn{6}{|l|}{$\%$ Poverty } \\
\hline$<100 \%$ & 9.9 & 13.4 & 9.2 & HUD subsidized & 77.7 \\
\hline $100-149 \%$ & 11.9 & 15.7 & 11.2 & Fair Market Rent & 22.3 \\
\hline $150 \%$ or $>$ & 78.2 & 70.9 & 79.6 & & \\
\hline $\begin{array}{l}\text { Median income } \\
\text { among adults } \geq 65\end{array}$ & $\$ 25,210$ & $\$ 25,834$ & $\$ 26,633^{*}$ & $\begin{array}{l}\text { Sec. } 8 \\
\text { Sec. } 236 \\
\text { FMR }\end{array}$ & $\begin{array}{r}69.6 \\
8.1 \\
22.3\end{array}$ \\
\hline
\end{tabular}

U.S. Census Bureau, American Fact Finder, 2007

* Kentucky Data Center, 2002 
$(8.4 \%)$ and state $(5.1 \%)$ population figures, as well as for the urban county $(12.5 \%)$ in which the CCRC was located. The rate was also higher than anticipated given that only about 10 to $12 \%$ of the total population of the CCRC was African American. No other racial or ethnic minorities were represented in the target population, except for a single Asian American resident who did not speak English.

While all residents met criteria for low-income, as defined by HUD, only $77.7 \%$ of residents qualified for subsidized housing, under Section 8 or Section 236 programs. The remaining $22.3 \%$ were classified as fair market rent (FMR) $(\$ 23,250$ to $\$ 33,000)$ and, while meeting HUD-defined criteria for low-income status, did not qualify for rent subsidies at the CCRC. FMR income levels reported by the CCRC on the whole were at or below median income levels for adults aged 65 or older. About $80 \%$ of the sample resided in independent living, while $20 \%$ received assisted living services, congruent with the proportion of residents receiving assisted living services in the retirement community. Slightly more than half of participants (51.1\%) self-reported good or very good health and an additional $41 \%$ reported fair health. Total numbers of chronic diseases reported ranged from 0 to 6 , with $68.8 \%$ reporting three or fewer chronic diseases.

\section{Pairwise Correlations among Related Variables}

Bivariate correlations were examined, comparing objective measures with participants' perceptions of income, functional level, perceived health, and successful aging and are displayed in Table 5. Income, defined for the study as CCRC rent levels, was contrasted with participants' perceived income levels, defined as: barely enough, enough to get by, or enough with some luxuries. 
Table 5

Pairwise Correlations of Related Variables

1. Correlations among Income-related Variables

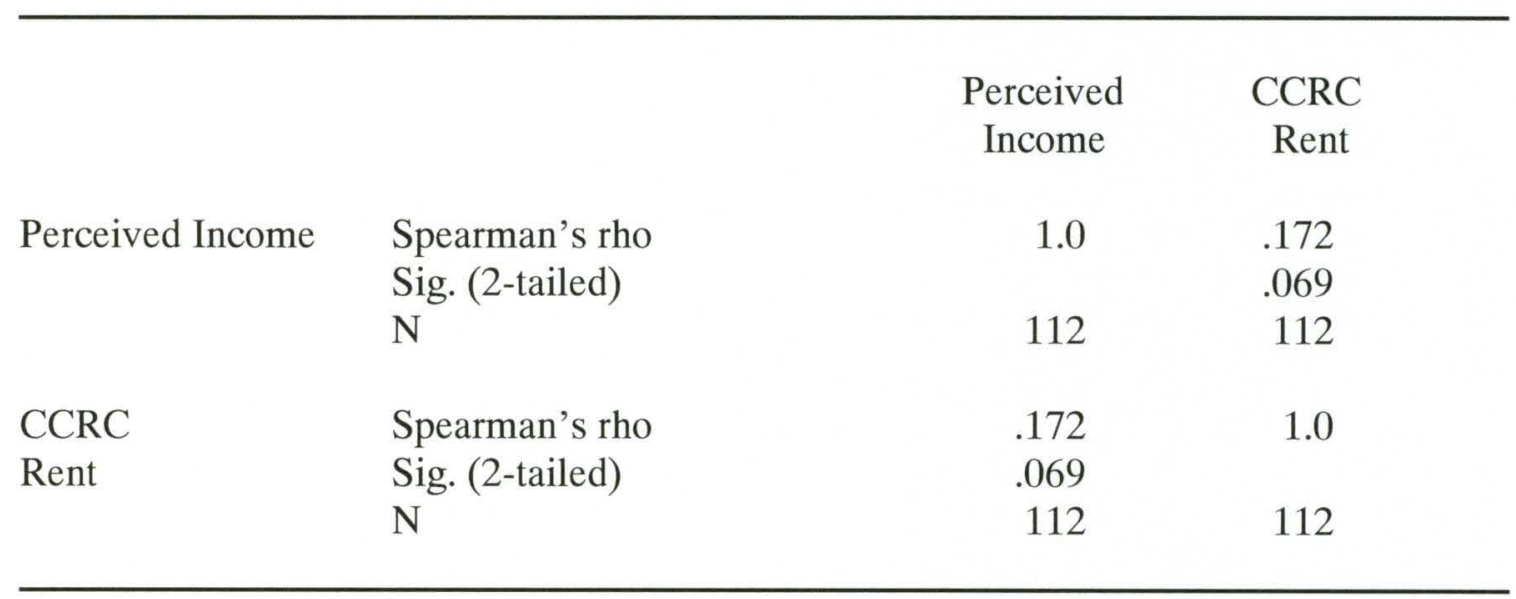

\section{Correlations among Function-related Variables}

Perceived CCRC

Function Function

Perceived Function Spearman's rho

Sig. (2-tailed)

$\mathrm{N}$

1.0

$.250 * *$

.008

$110 \quad 110$

CCRC

Function

Spearman's rho

Sig. (2-tailed)

$\mathrm{N}$
$.250 * *$

.008

110
1.0

110

** Correlation is significant at the 0.01 level (2-tailed) 


\section{Non-Parametric Tests of Correlations for Categorical Health-related Variables}

\begin{tabular}{llccc}
\hline & & $\begin{array}{c}\text { Perceived } \\
\text { Health }\end{array}$ & $\begin{array}{c}\text { Chronic } \\
\text { Disease }\end{array}$ & $\begin{array}{c}\text { Health } \\
\text { Index }\end{array}$ \\
Perceived health & $\begin{array}{l}\text { Spearman's rho } \\
\text { Sig. (2-tailed) }\end{array}$ & 1.0 & $.229^{*}$ & $.613^{* *}$ \\
& $\mathrm{~N}$ & 112 & .015 & .000 \\
Chronic Disease & Spearman's rho & 112 & 112 \\
& Sig. (2-tailed) & $.229^{*}$ & 1.0 & $.866^{* *}$ \\
& N & .015 & & .000 \\
Health index & Spearman's rho & 112 & 112 & 112 \\
& Sig. (2-tailed) & $.613^{* *}$ & $.866^{* *}$ & 1.0 \\
& N & .000 & .000 & 112 \\
\hline * Correlation is significant at the 0.05 level (2-tailed) & 112 & 112 & \\
** Correlation is significant at the 0.01 level (2-tailed) & & &
\end{tabular}

\section{Correlations among Perceived Successful Aging and Successful Aging Inventory}

\begin{tabular}{llcr}
\hline & & $\begin{array}{c}\text { Perceived } \\
\text { Successful Aging }\end{array}$ & SAI \\
Perceived Successful & Spearman's rho & 1.0 & -.081 \\
Aging & Sig. (2-tailed) & 112 & .401 \\
& $\mathrm{~N}$ & & 109 \\
Successful Aging & Spearman's rho & -.081 & 1.0 \\
Inventory (SAI) & Sig. (2-tailed) & .401 & 112 \\
& $\mathrm{~N}$ & 109 & \\
\hline
\end{tabular}

The correlation between income and perceived income was not statistically significant (Spearman's rho $=.17, p=.07$ ), although the positive coefficient indicated that as income level increased, participants did perceive a higher income level. Function, operationalized as the CCRC report of delivery and billing for either independent living or assisted living services, was significantly correlated with perceived function, i.e., participants' self- 
reported receipt of independent or assisted living services (Spearman's rho $-.25, p=.01$ ), as expected. The direction of the correlation indicated that as the number of residents receiving assisted living services increased, perceived functional level decreased. However, there was a statistically significant difference in the means between objective and perceived function $(F=7.22, \mathrm{df}=1,108, p=.008)$.

An objective measure of health, i.e., number of chronic health conditions, was significantly and positively correlated with perceived health (Spearman's rho $=.23, p=$ .015). A computed health index constructed by summing the values on perceived health and numbers of chronic disease, proposed to create a single health variable to preserve power, was also significantly correlated with both number of chronic conditions (Spearman's rho $=.866, p=.000)$ and with perceived health (Spearman's rho $=.613, p=$ .000). A Cronbach's alpha of .80 was obtained for the index. However, despite the apparent reliability of the index, because significance was found for the model with both health variables included independently and the index introduced concerns for validity, the index was subsequently not used in the analysis.

The association between scores on the SAI and participants' self-reported successful aging status was also examined, identifying a non-significant negative correlation (Spearman's rho $=-.08, p=.40$ ). The direction of the correlation indicated that as the SAI score increased, perceived successful aging decreased. The validity of this correlation must be questioned however, because of the extremely skewed distribution of participants' responses to the question on perceived successful aging ("Do you feel you are aging successfully?"). Among 109 responses, only about 5\% of participants answered negatively to the question. 


\section{Research Questions}

The next section of the analysis of the data focuses on four research questions explicated for the study. After examining results for each question, the analysis concludes with a report of reliability statistics for each of the three measurement instruments.

\section{Research question 1.}

The first research question asked if there were differences in successful aging, based on age, gender, race, marital status, education, income, function, perceived health, or total numbers of chronic disease. Differences were identified by examining both correlations and tests of differences in the means. First, bivariate correlations of SAI with demographic variables (age in years, gender, race, marital status, education) and with the five control variables (age in categories, income, function, perceived health, and number of chronic diseases) were examined. Then, differences in mean SAI scores among groups defined by age, gender, race, marital status, education, income, function, perceived health, or chronic disease were examined, using one-way analysis of variance (ANOVA).

In examining correlations, results revealed only two statistically significant correlations between SAI and demographic or control variables, as displayed in Table 6. Perceived health exhibited a statistically significant negative relationship with SAI (Spearman's rho $=-.217, p=.022$ ), although number of chronic diseases was not significantly correlated with SAI (Pearson's $r=.015, p=.88$ ). Also, while age in years 


\begin{tabular}{|c|c|c|c|c|c|c|c|c|c|c|}
\hline SAI & Age/yr. & Age/cat. & Gender & Race & Marital & Education & Income & Function & Per. Health & Chronic D \\
\hline SAI & -.182 & $-.22 *$ & .015 & -.091 & .015 & -.029 & -.034 & -.166 & $-217^{*}$ & .067 \\
\hline Sig. & .055 & .022 & .873 & .342 & .874 & .761 & .725 & .080 & .022 & .482 \\
\hline Age (years) & 1.0 & $.901 * *$ & -.225 & .039 & $.341^{*}$ & .013 & $.225^{*}$ & -.031 & .024 & $-.204^{*}$ \\
\hline Sig. & & .000 & .017 & .681 & .000 & .893 & .017 & .747 & .804 & .031 \\
\hline Age (categorical) & & 1.0 & -.101 & -.003 & .308 & .079 & .284 & -.024 & .103 & -.157 \\
\hline Sig. & & & .290 & .973 & .001 & .407 & .002 & .802 & .282 & .098 \\
\hline Gender & & & 1.0 & -.048 & $-.282 *$ & -.027 & .028 & -.140 & .019 & -.067 \\
\hline Sig. & & . & & .619 & .003 & .774 & .769 & .141 & .844 & .485 \\
\hline Race & & & & 1.0 & -.179 & -.173 & -.028 & -.139 & .004 & -.118 \\
\hline Sig. & & & & & .059 & .069 & .770 & .143 & .970 & .215 \\
\hline Marital & & & & & 1.0 & -.038 & -.161 & -.041 & .064 & .009 \\
\hline Sig. & & & & & & .694 & .090 & .671 & .502 & .927 \\
\hline Education & & & & & & 1.0 & .121 & .033 & .057 & .144 \\
\hline Sig. & & & & & & & .203 & .727 & .550 & .130 \\
\hline Income & & & & & & & 1.0 & -.001 & .052 & $-.200 *$ \\
\hline Sig. & & & & & & & & .989 & .583 & .035 \\
\hline Function & & & & & & & & 1.0 & -.079 & .044 \\
\hline Sig. & & & & & & & & & .407 & .649 \\
\hline Per. Health & & & & & & & & & 1.0 & $.229 *$ \\
\hline Sig. & & & & & & & & & & .015 \\
\hline $\begin{array}{l}\text { Chronic Dx. } \\
\text { Sig. }\end{array}$ & elation is & ficant at $t$ & .05 level (2 & ailed) & & & & & & 1.0 \\
\hline
\end{tabular}


was not significantly correlated with SAI (Pearson's $r=.23, p=.017$ ), age coded as a categorical variable (age 60-69= young-old, 70-79=old-old, $80+=$ oldest-old) $($ Garfein \& Herzog, 1995) demonstrated a weak, but statistically significant, negative correlation with SAI (Spearman's rho $=-.22, p=.022$ ). No other significant correlations were noted between successful aging (SAI) and any of the demographic or control variables, although the correlation with age in years and SAI $(r=-.18, p=.055)$ was of sufficient size to almost meet the .05 criterion of significance. The direction of the correlation indicated, as expected, that as age increased, successful aging scores decreased, but only among the oldest-old group.

Tests of differences in the means between successful aging and age (in years and as a categorical variable), gender, race, marital status, education, income, function, perceived health, and chronic disease were conducted next, using one-way analysis of variance, as displayed in Table 7 . None of the tests were statistically significant, and null hypotheses were retained for all variables.

Table 7 Differences in the Means between Successful Aging, Demographics, and Control Variables

$\begin{array}{lrrr}\text { Age in years } & .872 & 30,81 & .655 \\ \text { Age in categories } & 3.018 & 2,109 & .053 \\ \text { Gender } & .087 & 1,110 & .769 \\ \text { Race } & .717 & 1,110 & .399 \\ \text { Marital status } & .485 & 3,108 & .693 \\ \text { Education } & .345 & 3,109 & .793 \\ \text { Income } & .369 & 2,109 & .692 \\ \text { Function } & 2.303 & 1,110 & .132 \\ \text { Perceived health } & 1.836 & 3,108 & .145 \\ \text { Chronic disease } & .269 & 6,105 & .950 \\ \text { Health index } & .812 & 8,103 & .593\end{array}$


It was noted that age, coded categorically, did demonstrate a non-significant $F$-statistic $(F=3.018, \mathrm{df}=2,109, p=.053)$ that was close to the statistical significance criterion of .05 .

\section{Research question 2.}

The second research question asked what relationships existed among adaptation, operationalized as proactive coping (PCI), transcendence, measured as self-transcendence (STS), and successful aging, measured using Flood's (2008) recently developed Successful Aging Inventory (SAI). See Table 8 for statistics on correlations among the PCI, STS, and SAI. Bivariate correlations revealed that both proactive coping (Pearson's $r=.51, p=.000$ ) and self-transcendence (Pearson's $r=.66, p=.000)$ were statistically significantly correlated with successful aging. Considering the violation of the assumption of normality due to the inclusion of outliers, non-parametric tests were also conducted. The correlation of SAI with PCI remained significant (Spearman's rho $=.46$, $\mathrm{p}=.000$ ), as did the correlation between SAI and STS (Spearman's rho $=.66, \mathrm{p}=.000$ ), although coefficients were somewhat smaller. The direction of all correlations was positive, indicating that as coping and transcendence increased so too did successful aging. The strengths of the relationships were moderate to large (Cohen, 1988), with transcendence somewhat more strongly associated with successful aging than coping. It was also noted that the two predictor variables, coping and transcendence, were intercorrelated (Pearson's $r=.57, p=.000 ;$ Spearman's rho $=.52, p=.000$ ), introducing the possibility of multicollinearity. As reported below, regression analysis was used to further investigate the relative importance of the effects of coping and transcendence on successful aging and found both proactive coping and self-transcendence were significantly associated with successful aging. 
Table 8

Bivariate Correlations among Outcome and Two Predictors

1. Correlations among Successful Aging, Proactive Coping, and Self-transcendence

\begin{tabular}{lllll}
\hline & & & & \\
& & SAI & PCI & STS \\
\cline { 2 - 4 } SAI & Pearson Correlation & 1.0 & $.514^{* *}$ & $.666^{* *}$ \\
Successful Aging & Sig. (2-tailed) & & .000 & .000 \\
& $\mathrm{~N}$ & 112 & 112 & 112 \\
& & & & \\
PCI & Pearson Correlation & $.514^{* *}$ & 1.0 & $.567^{* *}$ \\
Proactive Coping & Sig. (2-tailed) & .000 & & .000 \\
& $\mathrm{~N}$ & 112 & 112 & 112 \\
STS & Pearson Correlation & $.666^{* *}$ & $.567^{* *}$ & 1.0 \\
Self-transcendence & Sig. (2-tailed) & .000 & .000 & 112 \\
& N & 112 & 112 & \\
\hline
\end{tabular}

2. Nonparametric Correlations among Successful Aging, Proactive Coping, and Self-transcendence

\begin{tabular}{|c|c|c|c|c|}
\hline & & $\underline{\text { SAI }}$ & $\mathrm{PCI}$ & STS \\
\hline SAI & Spearman's rho & 1.0 & $.460^{* *}$ & $.656^{* *}$ \\
\hline \multirow[t]{2}{*}{ Successful Aging } & Sig. (2-tailed) & & .000 & .000 \\
\hline & $\mathrm{N}$ & 112 & 112 & 112 \\
\hline PCI & Spearman's rho & $.460^{* *}$ & 1.0 & $.524^{* *}$ \\
\hline \multirow[t]{2}{*}{ Proactive Coping } & Sig. (2-tailed) & .000 & & .000 \\
\hline & $\mathrm{N}$ & 112 & 112 & 112 \\
\hline STS & Spearman's rho & $.656^{* *}$ & $.524^{* *}$ & 1.0 \\
\hline \multirow[t]{2}{*}{ Self-transcendence } & Sig. (2-tailed) & .000 & .000 & \\
\hline & $\mathrm{N}$ & 112 & 112 & 112 \\
\hline
\end{tabular}




\section{Research question 3.}

The third research question asked to what degree proactive coping and selftranscendence explained any observed variance in successful aging. The question also asked about the relative contributions of each predictor. To address this question successful aging was regressed on two continuous predictor variables, proactive coping and self-transcendence, controlling for age, income, function, perceived health and number of chronic health conditions. Income was re-coded as a binary variable (subsidized or non-subsidized), and functional status (independent or assisted) originated as a binary variable, to meet criteria for multiple regression. Age in years was dummy coded as two binary variables, i.e., old-old (aged $70-79$ ) and oldest-old (aged 80 and above), while young-old (aged $60-69$ ) served as the reference category. Perceived health was dummy coded as three dichotomous variables (very good, good, fair), as well as the reference category (poor).

Using hierarchical multiple regression analysis, variables were entered in two blocks, using Method Enter for each block. Hierarchical regression uses a theoretical basis to establish the order of entry for the two sets of predictors. The five control variables were entered together in the first block, and the two predictors were entered in the second block. Listwise deletion was used for missing values, with $\mathrm{N}=112$. See Table 9 for the report of the regression analyses.

The correlation matrix revealed that among the control variables age was statistically significantly correlated with SAI. For age 70-79, contrasted with age ranges 60 to 69 and age 80 or above, the correlation was small $(r=.16, p=.05)$; for age 80 and above, contrasted with age ranges 60 to 69 and 70 to 79 , the negative regression 
Table 9

Multiple Regression Analysis

\section{Descriptive Statistics}

\begin{tabular}{llll}
\hline & Mean & SD & N \\
SAI/Successful Aging & 62.53 & 9.321 & 112 \\
Age: $\quad \begin{array}{ll}\text { 70-79 } \\
\quad 80+\end{array}$ & .3661 & .4839 & 112 \\
& .5179 & .5019 & 112 \\
CCRC Rent, binary & 1.22 & .4183 & 112 \\
CCRC Function, binary & 1.21 & .406 & 112 \\
$\quad \begin{array}{l}\text { Perceived health: } \\
\quad \text { Very good }\end{array}$ & & & \\
$\quad$ Good & .0982 & .2989 & 112 \\
$\quad$ Fair & .4196 & .4957 & 112 \\
Chronic disease, continuous & .4107 & .4942 & 112 \\
$\quad$ & 2.85 & 1.296 & 112 \\
PCI/Proactive Coping & 41.09 & 6.873 & 112 \\
STS/Self-transcendence & 3.454 & .346 & 112 \\
\hline
\end{tabular}




\begin{tabular}{|c|c|c|c|c|c|c|c|c|c|c|}
\hline \multirow[b]{2}{*}{ Model } & \multirow[b]{2}{*}{$R$} & \multirow[b]{2}{*}{$R$-square } & \multirow[b]{2}{*}{$\begin{array}{c}\text { Adjusted } \\
\text { R-square }\end{array}$} & \multirow[b]{2}{*}{$\begin{array}{l}\text { Std. Error of } \\
\text { the Estimate }\end{array}$} & \multirow[b]{2}{*}{$\begin{array}{l}\text { R-square } \\
\text { Change }\end{array}$} & \multicolumn{4}{|c|}{ Change Statistics } & \multirow[b]{2}{*}{ Durbin-Watson } \\
\hline & & & & & & F-Change & $\mathbf{d f}_{1}$ & $\mathbf{d f}_{\mathbf{2}}$ & $\begin{array}{l}\text { Sig. of } \\
F-\text { Change }\end{array}$ & \\
\hline 1 & $.356^{\mathrm{a}}$ & .127 & .059 & 9.042 & .127 & 1.869 & 8 & 103 & .073 & \\
\hline 2 & $.709^{b}$ & .503 & .454 & 6.890 & .376 & 38.204 & 2 & 101 & .000 & 1.793 \\
\hline
\end{tabular}

a Predictors: (Constant), Age, Perceived Health, \# of Chronic Dx., CCRC Rent, CCRC Function

b Predictors: (Constant), Age, Perceived Health, \# of Chronic Dx., CCRC Rent, CCRC Function, Proactive Coping Inventory, Self-transcendence Dependent: Successful Aging Inventory

\section{ANOVA}

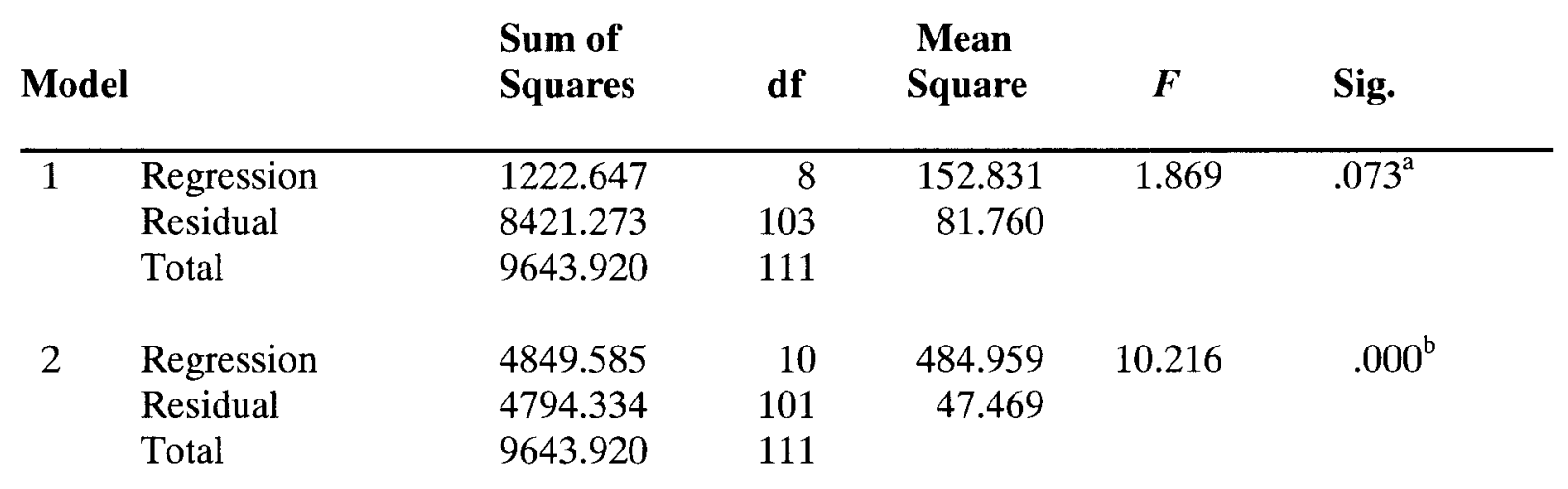

Predictors: (Constant), Age, Perceived Health, \# of Chronic Dx., CCRC Rent, CCRC Function

b Predictors: (Constant), Age, Perceived Health, \# of Chronic Dx., , CCRC Rent, CCRC Function, Proactive Coping Inventory, Self-transcendence Dependent: Successful Aging Inventory 


\section{Regression Coefficients}

\begin{tabular}{|c|c|c|c|c|c|c|c|c|c|c|}
\hline \multirow[t]{2}{*}{$\overline{\text { Model }}$} & \multicolumn{2}{|c|}{$\begin{array}{l}\text { Unstandardized } \\
\text { Coefficients } \\
\end{array}$} & \multicolumn{3}{|c|}{$\begin{array}{r}\text { Standardized } \\
\text { Coefficients }\end{array}$} & \multicolumn{3}{|c|}{ Correlations } & \multicolumn{2}{|c|}{$\begin{array}{r}\text { Collinearity } \\
\text { Statistics } \\
\end{array}$} \\
\hline & B & Std. Error & Beta & $t$ & Sig. & Zero-order & Partial & Part & Tolerance & VIF \\
\hline 1. (Constant) & 64.642 & 5.737 & & 11.267 & .000 & & & & & \\
\hline Age, 70 - 79 & -1.806 & 2.930 & -.094 & -.616 & .539 & .157 & -.061 & -.057 & .367 & 2.728 \\
\hline Age $80+$ & -4.758 & 2.895 & -.256 & -1.644 & .103 & -.226 & -.160 & -.151 & .349 & 2.866 \\
\hline CCRC Rentdi & -.008 & 2.153 & -.000 & -.004 & .997 & -.077 & -.000 & -.000 & .908 & 1.101 \\
\hline CCRC Function & -3.936 & 2.145 & -.171 & -1.835 & .069 & -.143 & -.178 & -.169 & .972 & 1.028 \\
\hline Very good Per. Health & 7.375 & 4.370 & .237 & 1.688 & .095 & 143 & .164 & .155 & .432 & 2.137 \\
\hline Good Per. Health & 5.208 & 3.481 & 277 & 1.496 & .138 & .117 & .146 & .138 & 247 & 4.042 \\
\hline Fair Per. Health & 1.724 & 3.481 & .091 & .495 & .621 & -.157 & .049 & .046 & .249 & 4.017 \\
\hline Chronic Disease & .747 & .724 & . 104 & 1.031 & .305 & .067 & .101 & .095 & .836 & 1.197 \\
\hline 2. (Constant) & 8.337 & 7.900 & & 1.055 & .294 & & & & & \\
\hline Age, $70-79$ & -2.721 & 2.260 & -.141 & -1.204 & .231 & .157 & -.119 & -.084 & 358 & 2.797 \\
\hline Age, $80+$ & -4.105 & 2.228 & -.221 & -1.843 & .068 & -.226 & -.180 & -.129 & .342 & 2.923 \\
\hline CCRC Rent-di & -.277 & 1.659 & -.012 & -.167 & .868 & -.077 & -.017 & -.012 & .888 & 1.126 \\
\hline CCRC Function & -2.538 & 1.678 & -.110 & -1.512 & .134 & -.143 & -.149 & -.106 & .922 & 1.085 \\
\hline Very good Per. Health & 7.375 & 4.370 & .237 & 1.688 & .095 & .143 & .164 & .155 & 432 & 2.317 \\
\hline Good Per. Health & 5.208 & 3.481 & .277 & 1.496 & .138 & 117 & 146 & .138 & .247 & 4.042 \\
\hline Fair Per. Health & 1.724 & 3.481 & .091 & .495 & .621 & -.157 & .049 & .046 & .249 & 4.017 \\
\hline PCI & .270 & .122 & .199 & 2.219 & $.029^{*}$ & .514 & .216 & .156 & .612 & 1.633 \\
\hline STS & 14.059 & 2.484 & .523 & 5.659 & $.000^{*}$ & .666 & .491 & .397 & .577 & 1.733 \\
\hline
\end{tabular}

Dependent Variable: SAI Successful Aging Inventory 
correlation was also small $(r=-.23, p=.008)$. (The bivariate correlation of SAI with ages 60 through 69 , not included in the regression model, was non-significant and positive, i.e., Spearman's rho $=.98, p=.302$ ).

Health was not significantly correlated with SAI; i.e., fair health, contrasted with very $\operatorname{good}(r=.143, p=.06)$, or with good health $(r=.117, p=.11)$, was weakly and negatively correlated $(r=-.157, p=.05)$ with SAI. (Bivariate correlation of SAI with poor perceived health, not included in the regression model, was also non-significant and negative. i.e., $r=-.09, p=.343$ ). Proactive coping was significantly and moderately correlated with SAI $(r=.51, p=.000$ as was self-transcendence $(r=.67, p=.000)$. Proactive coping was also significantly correlated with self-transcendence $(r=.57, p=$ .000 ), indicating the potential for multicollinearity, which was not unexpected for the present analysis.

The first model $\left(\mathrm{R}^{2}=.127\right.$ and Adj. $\left.\mathrm{R}^{2}=.059\right)$, in which only the control variables were entered, was not statistically significant $(F=1.869 . \mathrm{df}=8,103, p=.073)$ and explained only about $6 \%$ of the variance in successful aging. The addition of coping and transcendence in the second model explained an additional $38 \%$ of the variance, resulting in an $R^{2}$ of .50 and an Adjusted $R^{2}$ of .454 . The second model, when PCI and STS were entered, was statistically significant $(F=10.216, \mathrm{df}=10,101, p=.000)$ and was interpreted as explaining $45.4 \%$ of the variance in successful aging. As previously discussed, in regression analysis not only is statistical significance of importance, but also the ability to apply the model to successive samples of the same population in future. To increase the probability of the model replicating without undue shrinkage of the coefficient of determination $\left(\mathrm{R}^{2}\right)$, the regression was interpreted conservatively, using the 
adjusted $\mathrm{R}^{2}$. Thus it was concluded, in answer to the third research question, that proactive coping and self-transcendence together accounted for $45.4 \%$ of the variance in successful aging, rather than interpreting the raw coefficient of determination $\left(\mathrm{R}^{2}=.50\right)$.

The regression coefficients were then examined to determine if both predictors contributed to the model, and to assess the relative contribution of each significant predictor. Beta weights for all control variables were non-significant; control variables did not contribute significantly to either the first model, in which only control variables were entered, or to the final model including the two predictors. When the two predictors, proactive coping and self-transcendence, were added in the second model, both contributed to the model, as indicated by statistically significant unstandardized regression coefficients of $.27(t=2.22, p=.029)$, and $14.059(t=5.659, p=.000)$ respectively.

Standardized regression coefficients for the predictors were then examined to evaluate the relative contributions of proactive coping and self-transcendence. The standardized coefficient for proactive coping $(\beta=.199)$ was smaller than the standardized coefficient for self-transcendence $(\beta=.523)$. The standardized coefficient may be interpreted as the change in successful aging for every one standard deviation change in the predictor. Based on the ratio of Betas for proactive coping and transcendence, the effect of transcendence on successful aging is about 2.5 times greater than the effect of proactive coping. Thus while proactive coping was an important predictor of successful aging, self-transcendence was the more important predictor. The Beta values made it apparent that transcendence served as a significant predictor of successful aging, 
independent of the effects of proactive coping and accounted for a greater proportion of the variance in successful aging.

The regression analysis was completed by verifying that assumptions for regression were met. See Table 10 for regression diagnostics and Figure 3 for plots related to regression assumptions. Normality was established by a histogram (Figure 3a) which was symmetric and exhibited a normal curve with a single peak. Linearity was clearly exhibited when expected probabilities were plotted against observed probabilities (Figure 3b). A scatterplot of residuals (vertical axis) plotted against standardized predicted values (horizontal axis) demonstrated a random cluster of datapoints equally distributed above and below a horizontal line crossing zero, meeting the assumption for homoscedasticity (Figure 3c).

Table 10

Regression Diagnostics

\begin{tabular}{lccccc}
\hline & Minimum & Maximum & Mean & SD & N \\
\hline Predicted Value & 39.67 & 73.91 & 62.53 & 6.61 & 112 \\
Std. Predicted Value & -3.458 & 1.72 & .000 & 1.00 & 112 \\
Std. Error of & & & & & \\
Predicted Value & 1.367 & 3.755 & 2.105 & .481 & 112 \\
Adjusted Pred.Value & 42.75 & 74.02 & 62.53 & 6.66 & 112 \\
Residual & -20.53 & 15.59 & .000 & 6.57 & 112 \\
Std. Residual & -2.98 & 2.262 & .000 & .954 & 112 \\
Stud. Residual & -3.18 & 2.376 & .000 & 1.00 & 112 \\
Deleted Residual & -23.30 & 17.19 & -.001 & 7.29 & 112 \\
Stud. Deleted Residual & -3.33 & 2.433 & -.002 & 1.02 & 112 \\
Mahal. Distance & 3.377 & 31.97 & 9.91 & 5.18 & 112 \\
Cook's Distance & .000 & .123 & .010 & .018 & 112 \\
Centered Leveraged & .030 & .288 & .089 & .047 & 112 \\
\hline a Dependent Variable: TotalSAI Total Successful Aging Score & & \\
\hline
\end{tabular}


Figure 3

\section{Plots Related to Regression Assumptions}

\section{A. Histogram}

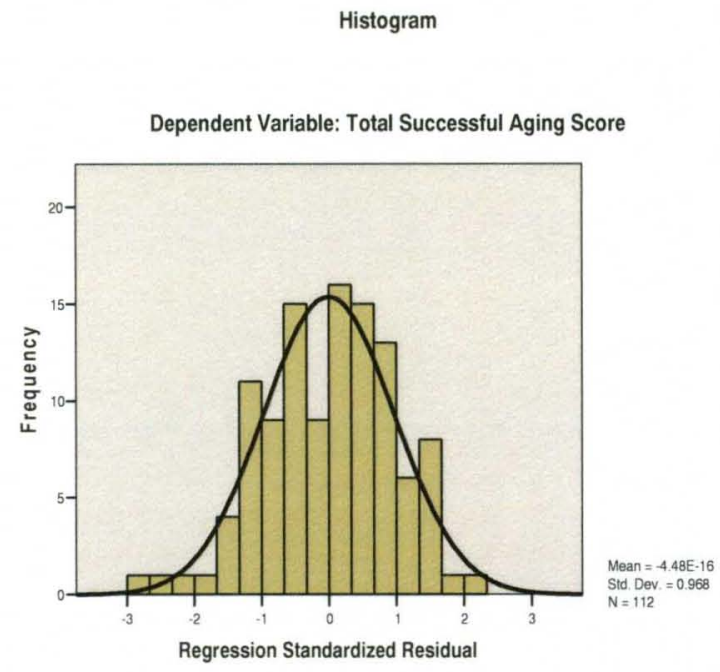

B. Normal P-P Plot

Normal P.P Plot of Regression Standardized Residual

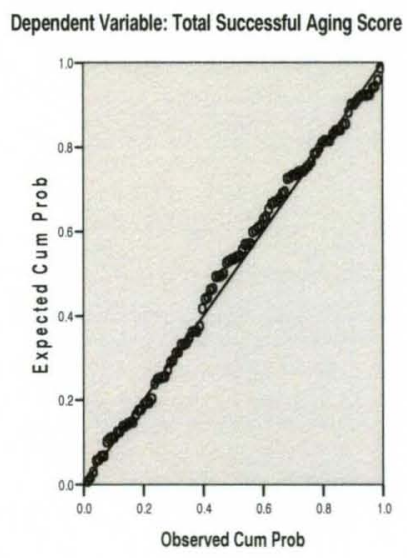

D. Boxplot

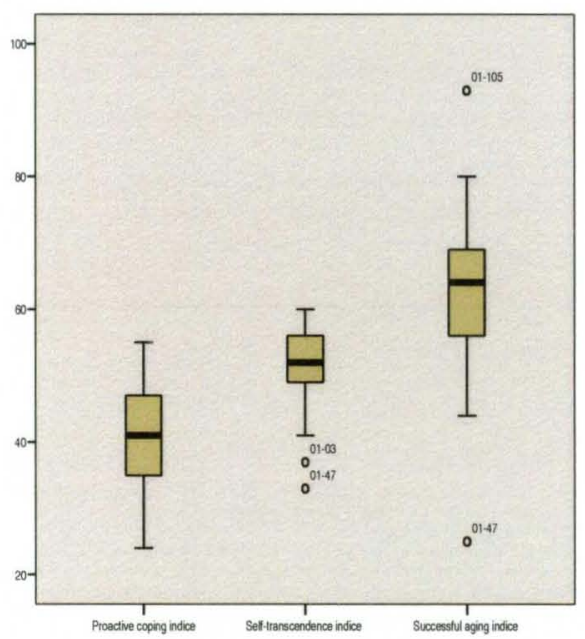


The Durbin-Watson test for the correlation of adjacent residuals confirmed the assumption of independence was met as the value of 1.793 was close to 2 , in a range of 0 to 4 . Noting the significant bivariate correlation between proactive coping and selftranscendence $(r=.57, p=.000)$, the value fell below the limit of .70 , suggesting multicollinearity was not a problem. Multicollinearity was further assessed by examining statistics for Tolerance and the Variance Inflation Factor (VIF). Tolerance (.238 to .922) and VIF (1.085 to 4.196) statistics were consistent with data that do not have a serious violation of multicollinearity. Despite four outliers revealed on the boxplot (Figure 3d), casewise diagnostics revealed no datapoint exceeded the range of three standard deviations from the mean. Mean standardized and studentized residuals were both .000 . Cook's D was .010, less than 1.0. Centered leveraged value (.089) was below the computed cut-off value of .125 .

Given the above tests, it was concluded that assumptions for multiple regression were met and the model, with an adjusted coefficient of determination of .45, was likely to predict not only a significant amount of the variance in SAI, but also a practically or clinically significant amount. Additionally, the effect size indicates goodness-of-fit adequate to replicate accurately without undue shrinkage in future samples from the population.

\section{Research question 4.}

The final research question asked if there were differences observed in proactive coping, self-transcendence, and successful aging between those participants who responded positively to the dichotomous question, "Do you feel you are aging successfully?" and to those who responded they were not aging successfully, with binary 
logistic regression used to analyze the results. However, among the final sample of 112 participants, $103(92 \%)$ responded that they were aging successfully, and only $6(5.4 \%)$ reported they were not aging successfully, with $3(2.7 \%)$ missing values. Thus the conclusion was reached that there was not sufficient variance in the data to enable a meaningful analysis and this question was not answered for the study.

\section{Reliability Statistics}

Analysis was conducted to obtain reliability statistics for each of the three measurement scales used in the study: the Proactive Coping Subscale of the Proactive Coping Inventory (Greenglass, 1999, 2002), the Self-transcendence Scale (Reed, 1989b), and the Successful Aging Inventory (Flood, 2008). Reliability statistics are provided in Table 11. The Cronbach's alpha for the Proactive Coping Subscale (Greenglass, 1999, 2002) (.77) and for the Self-transcendence Scale (Reed, 1989b) (.73) were acceptable as each exceeded the minimum of .70. The alpha for the Successful Aging Inventory (Flood, 2008) (.82) was somewhat stronger.

A reliability statistic was also obtained for the computed health index, used as a composite measure of perceived health and self-reported number of chronic diseases. The Cronbach's alpha of .80 suggested the index, as a composite measure of health merging self-reported health $(1=$ very good, $2=\operatorname{good}, 3=$ fair, $4=$ poor $)$ with total number of chronic conditions, may merit further study in future. 
Table 11

Reliability Statistics for Measurement Scales

\begin{tabular}{lccc} 
& Cronbach's Alpha & $\begin{array}{l}\text { Cronbach's Alpha } \\
\text { Based on Std. Items }\end{array}$ & N of items \\
\hline Proactive Coping Subscale & .77 & .80 & 14 \\
Self-Transcendence Scale & .73 & .75 & 15 \\
Successful Aging Inventory & .82 & .83 & 20 \\
Health Index & .80 & .81 & 3 \\
\end{tabular}




\section{CHAPTER V}

\section{DISCUSSION}

Chapter five begins with a summary of the dissertation study, including an overview of the problem and a review of the purpose of the study, research questions, and methodology. Next results related to data collection and sample size are reported. Then major findings related to each research question are discussed and interpreted in light of the literature. Next conclusions are specified, with consideration of limitations of the study and recommendations for further research. The chapter ends with concluding remarks.

Overview of the Problem, Purpose and Research Questions

The overarching goal of the study was to examine Flood's (2005) mid-range nursing theory of successful aging for potential usefulness as a guide to a more holistic view of aging, particularly among disadvantaged older adult populations. Current successful aging theory originated largely from the medical model and focused almost entirely on behavioral determinants of health. Little research was identified that considered how older adults themselves defined successful aging (Phelan \& Larson, 2002; Phelan et al., 2004; Strawbridge, et al. 2002). The few studies which did explore how older adults perceived successful aging identified two areas not previously noted; 
these were: (a) social and environmental determinants of health (Holstein \& Minkler, 2003; Knickman \& Snell, 2002; Schulz \& Northridge, 2004), and (b) spiritual or existential factors such as a sense of meaning or purpose in life, generativity, and connectedness with the self, others, and a higher power (Bowling \& Dieppe, 2005; Bowling \& Iliffe, 2006; Depp \& Jeste, 2006; Sadler \& Biggs, 2006). Nursing's ability to understand older adults' personal goals and priorities and to effectively support coping efforts older adults use to achieve successful aging was limited by a lack of appropriate theory (Flood, 2005; Wadensten \& Carlsson, 2003). Thus the problem addressed in this study was the need for comprehensive, multidimensional nursing theory, both to improve positive, quality aging for older adults and their caregivers, and to reduce the potential burden on society resulting form the unprecedented numbers of older adults in the total population.

The study's primary purpose was to explore Flood's (2005 Theory of Successful Aging, which proposed that adaptation and gerotranscendence predict a new perspective on successful aging, defined by the individual older adult rather than through normative standards defined by researchers. A descriptive, cross-sectional survey design was used, with a stratified random sample of older adults, living independently or receiving assisted living services, in a non-profit, low-income CCRC located in an urban county of a Southern state. The first research question asked if there were differences in successful aging related to age, gender, race, marital status, education, health, income level, or functional status. The second and third research questions asked whether both adaptation and transcendence were significant predictors of successful aging, and if so, what were the relative contributions of each predictor. A final research question asked whether there 
were differences in levels of adaptation, transcendence, and successful aging among those who reported they were aging successfully and those who reported they were not aging successfully.

Major findings from the study supported Flood's (2005) Theory of Successful Aging. Both constructs proposed in the theory, i.e., adaptation, operationalized for the study as proactive coping, and gerotranscendence, operationalized as self-transcendence, were significant predictors of successful aging defined from the individual older adult's perspectives. Together, adaptation and transcendence accounted for $45.4 \%$ of the variance of successful aging detected among the study sample, independent of the effects of age, health, income level, or functional status. The study also found that $92 \%$ of participants perceived they were aging successfully, regardless of age, health, income level, functional status, or objective measures on the Successful Aging Inventory (Flood, 2008). This section of the dissertation first briefly discusses aspects of the study related to data collection and characteristics of the sample, followed by conclusions reached for each of the research questions, then a discussion of the major findings, considered limitations of the study, and suggested directions for future research.

\section{Data Collection}

A stratified, random sample of non-demented adults aged 65 or older $(\mathrm{N}=112)$, living independently or receiving assisted living services in a low-income continuing care retirement community (CCRC) located in an urban county of a Southern state was recruited. The sample was selected from a sampling frame providing contact information, functional status, and rent levels for all residents of the CCRC. Residents were given the opportunity to opt out of inclusion in the sampling frame; no residents chose to do so. 
Although the population of the CCRC was originally estimated at 392 residents, the actual sampling frame was smaller due to a renovation program resulting in an unusually high vacancy rate. Considering that $20 \%$ of apartments were vacant and 46 additional residents were temporarily absent or unable to participate due to illness or disability, 261 residents were eligible for recruitment, one third fewer than anticipated. All but $22(8.4 \%)$ of the eligible target population were successfully contacted by telephone. Among the 239 eligible residents of the CCRC who were contacted and invited to participate, 123 participants were guided through the informed consent process, for a response rate of $51.5 \%$, exceeding the estimated response rate of $33 \%$. Ten $(8.1 \%)$ cases were subsequently excluded because they did not meet study criteria based on age 65 or greater and a normal Mini-Cog Dementia tests score, less than the projected $10 \%$ level for exclusions. One additional case was subsequently excluded due to excessive missing values, for a final sample size of 112 .

Residents who declined to participate gave reasons including lack of time, not feeling well, or unwillingness to answer questions about themselves. Beyond the probability that residents who were aging less successfully were more likely to decline, some participants may have declined due to concerns about revealing income and functional needs which could affect rent and fees, according to CCRC social services staff. Other residents $(n=9)$ rescinded their agreement after receiving the Consent and HIPAA Authorization documents in advance of data collection, reporting concerns for privacy due to the length and wording of the documents. Subsequently the study protocol was amended and approved by the university IRB to present the Consent and HIPAA documents at the beginning of each data collection session rather than at the time of 
scheduling, while the investigator was present to explain and answer questions immediately. No further sessions were cancelled due to concern over study documents.

Three elements of data collection used in the present study were not previously identified in the literature: (a) Administering surveys to small groups ( $n=5$ to 7 ) rather than one-on-one sessions; (b) Including all participants who gave consent in data collection sessions, while later excluding data based on abnormal scores on the Mini-Cog dementia tests; and (c) Accommodating sensory or literacy deficits by using large print and reading all items aloud twice.

The use of small groups was previously examined in a feasibility study (McCarthy, 2009), determining group size and identifying potential problems with space considerations which were addressed in the present study. Thus data collection sessions were conducted in a comfortable, private space with sufficient tables and chairs to seat participants well apart from each other. Using the standardized protocol, participants were asked to refrain from talking or reacting in any way to survey items so as not to influence other participants' responses. It was emphasized that there were no right or wrong answers and that each participant's unique perspective, experiences, and opinions were highly valued. Participants, when questioned following data collection, stated rather than feeling inhibited by the need to remain quiet they instead felt reassured about providing frank responses themselves and not feeling the need to provide socially acceptable answers. Data were collected in 24 sessions with an average of 5 participants per session. Number of participants ranged from two to eight per session. One session included eight participants while five sessions were conducted with only two or three participants, due to last minute cancellations or no shows. 
A second question addressed in the feasibility study (McCarthy, 2009) was how to identify and exclude participants who did not meet the criteria intended to achieve a non-demented sample. The feasibility study supported the use of the Mini-Cog Test as an effective screen for dementia and determined it was possible to administer the Mini-Cog at the beginning of data collection after informed consent was obtained, allow all participants to complete the survey process, regardless of Mini-Cog score, and then subsequently exclude surveys with abnormal Mini-Cog scores from the analysis. The feasibility study identified the potential for disruptive or distracting behavior from older adults experiencing symptoms of dementia. To accommodate for this problem, the present study scheduled participants by telephone, then delivered a written reminder at least 24 hours in advance of data collection. Because one of the earliest signs of the onset of dementia tends to be loss of executive function, such as keeping track of appointments (Johnson, Lie, \& Yaffe, 2007), no further reminders or follow-up was done for residents who did not keep scheduled appointment. The result was that with few exceptions residents with dementia did not attend the data collection session. Among the eight (6.4\%) consented participants who did attend data collection but were subsequently excluded due to abnormal Mini-Cog scores, none exhibited any distracting or disruptive behaviors, although CCRC staff was immediately available if needed.

One additional case was of interest. The single case excluded from the analysis due to excessive missing values had a normal Mini-Cog score, although examination of the survey showed obvious difficulty following the survey process. The Mini-Cog Test consists of recall on three words and completion of a clock face. Criteria for a normal score are either recall of all three words or recall of one or two words with a correct clock 
face. The participant completing the survey with excessive missing values recalled three words, thus achieving a normal Mini-Cog score. However, the clock face was grossly abnormal. Had the participant recalled only one or two words, the clock face would have resulted in an abnormal Mini-Cog score and exclusion from the study. It was interesting to note that the clock face included executive function, the specific cognitive ability involved in planning and execution of goal-directed behaviors, abstract reasoning, and judgment involved in completing the survey, rather than memory alone (Borson, 2000; Esteban-Santillan, Praditsuwan, Ueda, Geldmacher, 1998). It is possible that the clock face test alone might be sufficient for future studies. It is also possible that scoring on the Mini-Cog should be reconsidered in light of current studies on frontal lobe dementias (Woodford \& George, 2007).

A third element of data collection involved strategies designed to include older adults with sensory or literacy deficits. These included clear written directions on surveys, use of 14-point font, enlarged versions of each scale in 24-point font, and reading each item aloud at least twice, taking care to face participants and speak clearly in a low pitched voice appropriate for limited hearing or use of lip-reading. To maintain a standardized protocol, all data collection sessions, even those with only two or three participants who obviously did not require accommodation, included these strategies. Many residents expressed appreciation for being asked their opinions, stating that they enjoyed the process, although a few complained that for participants without sensory deficits the process of reading each question aloud twice was tedious, but not unacceptable. It was concluded that the benefit of including participants with sensory or 
literacy deficits in the study outweighed the burden on participants without limitations, as participants themselves stated.

\section{Characteristics of the Sample}

On the whole, the sample was representative of the target population. Statistics for mean age, gender, and marital status were as expected, considering the typical older adult in a CCRC is an eighty year old widow (Harris-Kojetin, Joseph, Zimring, \& Keifer, 2005). Ages of participants ranged from 65 to 95 , with a mean age of 80 . Women made up slightly more than $80 \%$ of the sample; about half of participants were widowed, and only $10 \%$ were married. The number of African Americans consenting to participate in the study exceeded the proportion of African Americans in the population of the CCRC (10 to $12 \%$ ), as well as the number of African Americans among the general older adult population (U.S. Census, 2007). It is possible that African Americans' experience of racial discrimination earlier in the life course influenced the propensity for social action in later life, such as participating in a study aimed at informing social and health policies for older adults (Ferraro \& Shippee, 2009). Thomas and Quinn (1991), however, noted the history of limited participation of African Americans in research studies, secondary to the Tuskegee Syphilis study. Other studies have found that African Americans were willing to participate if they had reason to trust the researcher (Kressin, Meterko, \& Wilson, 2000; Shavers-Hornaday, Lynch, Burmeister, \& Torner, 1997). In the present study, the investigator concluded that residents of the CCRC had established trust in the administration of the CCRC over time which was transferred to the research study, as a result of staff support for participation in the study. This relationship would appear to merit further study. 


\section{Sample characteristics related to health.}

The number of chronic diseases reported by participants ranged from 0 to 6 , with a mean of $2.85(\mathrm{SD}=1.30)$. Slightly more than half of participants reported their perceived overall health was good or better, with $93 \%$ reporting their health as at least fair. Scores on the health index ranged from 1 to 9 , with a mean score of 5.23. Significant pairwise correlations among perceived health, number of chronic diseases, and the composite health index and a Cronbach's alpha of .80 together provided support for the reliability of the health index. Strong support for the use of both perceived health and number of chronic diseases as indicators of health was found in the literature. Idler and Benyamini (1997), in a systematic review of 27 studies reported that 18 studies included both self-reported health and chronic diseases as indicators of health. Separately, perceived general or overall health as an accurate measure of health was well-established in the literature (Borawski, Kinney, \& Kahana, 1996; Idler \& Benyamini, 1997; Litwin \& Sapir, 2009; Rahman \& Barsky, 2003), as was the predictive power of the number of chronic diseases (Idler \& Benyamini, 1997; Jonnalagadda \& Diwan, 2005; Litwin \& Sapir, 2009). A combination of both variables in a single health index however was not

previously validated. For this reason the regression analysis was repeated with perceived health and number of chronic diseases as separate control variables without significant changes in the model. Given these results, the two separate health variables were included in the analysis, rather than the health index.

Sample characteristics related to income.

All residents of the CCRC met HUD-defined criteria for low-income status (less than $\$ 33,250$ annual income), but only $78 \%$ received subsidized rents; the remainder on 
average had incomes at or below median income levels for the general older adult population. Data analysis revealed objective income, as defined by HUD rent levels, was not associated with participants' perceived income. This was consistent with findings of a recent study $(\mathrm{N}=28,939)$ which concluded that older adults in the oldest-old category (aged 80 and above), as defined by Garfein and Herzog (1995), were likely to underestimate financial difficulties, even in the face of objective measures of financial distress (Litwin \& Sapir, 2009). It is possible that a sample drawn from a moderate or upper-income target population might have a significant correlation between actual income and perceived income. The lack of correlation between perceived income and objective income found in the present study may be relevant only to a low-income sample or could be due to the fact that older adults may be less aware of their income level if families handle the older adult's financial affairs.

Sample characteristics related to function.

Congruent with the makeup of the target population, about $80 \%$ of participants were classified by the CCRC as independent, defined as assistance with only instrumental activities of daily living, while $20 \%$ received one or more assisted living services, such as help with personal care, supervision of self-administered medications, or assistance with mobility. While only $80 \%$ of participants were classified as independent by the CCRC, $89 \%$ of participants self-reported independent function, a statistically significant difference between objective and perceived function. Considering that the question on perceived function asked specifically about being assigned to independent or assisted living, a discrepancy of $9 \%$ was unexpected. If anything, it was anticipated that more people would self-report needing assistance, reflecting sources other than the CCRC. Von 
Faber and his colleagues (2001) discussed the way older adults perceived their own functional ability, noting that self-assessment included comparison with others and adequacy of resources. It is possible the social and environmental resources and the comparison with other residents with multiple limitations influenced perceived function.

A similar disparity involved the contrast between successful aging, objectively measured on the SAI, and participants' perceived assessments of their own successful aging. Ninety-two percent of participants stated they were aging successfully, which was not correlated with objective successful aging as measured by the Successful Aging Inventory. The discrepancy between older adults' perceptions of successful aging and objective measures of successful aging might suggest an overall optimistic outlook, similar to that noted by Litwin and Shippee (2009) for perceived income adequacy, or may indicate the effects of factors associated with successful aging that were undetected in the study.

\section{Discussion of Findings Related to Four Research Questions}

Four specific research questions were addressed. The results of data analysis for each individual question will be discussed and interpreted in the next section of the report.

\section{Research question 1.}

The first research question asked if there were differences in successful aging, as measured by the Successful Aging Inventory, related to age, gender, race, marital status education, health, income, or functional status. Differences were examined by looking at correlations as well as tests of differences in the means between groups. There were few, if any, meaningful correlations noted between successful aging and age, gender, race, 
marital status education, health, income, or functional status, beyond a weak correlation between SAI and age 80 and older, contrasted with younger ages. This correlation between age and successful aging involved regression correlations for age defined categorically by Garfein and Herzog (1995), i.e., young-old (60 - 69), old-old (70 - 79), and oldest-old (80 and older).

While the correlation between SAI and the young-old category was not significant (Spearman's rho $=.98, p=.302$ ), the positive correlation of SAI with the old-old category (Pearson's $r=.16, p=.05$ ), although small, was statistically significant. The strength of the correlation increased slightly for the oldest-old (Pearson's $r=-.23, p=.008$ ) category, and also changed direction. This was interpreted as meaning that age was not related to successful aging among the young-old, but did become a significant - and positive factor - for the old-old; that is, increased age was associated with greater successful aging for those aged 70 to 79 . The change in direction for the correlation of SAI with age for the oldest-old indicated however that after age 80 , increased age was associated with a decrease in successful aging.

While the literature supported the absence of differences in successful aging based on gender, race, marital status, education, health, income, and function, the literature supported the relationship between successful aging and age for adults in very late life observed in the present study. One study of successful aging related to adaptation found a significant negative correlation between the oldest-old age group, versus other age groups (Freund \& Baltes, 1998). Freund and Baltes hypothesized that the decrease in successful aging with very late life was based on increasing frailty, dependency, and 
depletion of resources in late life, limiting coping options and ability to adapt to cumulative changes over time.

The lack of significant correlations of successful aging with health, income, and function was congruent with evidence from the few studies that have investigated successful aging defined from the perspective of older adults, rather than by investigatorimposed definitions, compared to normative criteria (Bowling \& Iliffe, 2006; Phelan, et al., 2004; Strawbridge et al, 2002). It is also possible these findings were limited to the specific setting and sample. Further studies of low-income populations in other settings as well as studies among older adults in CCRCs serving a wider range of income levels are needed before conclusions can be generalized outside a low-income CCRC.

Specifically, the relationship between advanced age and successful aging merits further investigation.

Finally, differences in SAI based on age, gender, race, marital status education, health, income, or functional status were examined by testing differences in the means, using one-way ANOVA. No significant differences in the means were identified.

\section{Research Question 2}

The second research question asked what relationships might exist among adaptation, operationalized as proactive coping (PCI), transcendence, measured as selftranscendence (STS), and successful aging, measured using Flood's Successful Aging Inventory (SAI). Statistically significant bivariate correlations among the three variables supported the conclusion that both proactive coping and transcendence were moderately to strongly associated with successful aging. Both proactive coping (Pearson's $r=.51, p=$ .000 ) and self-transcendence (Pearson's $r=.66, p=.000$ ) were positively correlated with 
successful aging. The nature of these relationships and the relative importance of the two predictors was considered in research question three, using multiple regression to address the question.

\section{Research Question 3}

The third research question asked to what degree adaptation and transcendence explained any observed variance in successful aging, controlling for age, income, health, and functional status. Hierarchical multiple regression produced a significant model predicting the effects of proactive coping and self-transcendence on successful aging, independent of age, health, income, and function. Age, health, income, and function, as operationalized for the study, together explained less than $3 \%$ of the variance.

When proactive coping and self-transcendence were added, the model accounted for $45.4 \%$ of the variance in successful aging. The size of the effect was sufficient to expect the model to replicate without undue shrinkage, and to be useful in future studies of successful aging in samples drawn from the same population. The effect size also suggested that the model is not only statistically significant but also of practical significance. That is, the effect of coping and transcendence was sufficiently large that interventions aimed at increasing one or both constructs are likely to produce an increase in successful aging among non-demented older adults residing in a low-income CCRC in an urban county of a Southern city.

It should be noted, however, that the target population encompassed a limited number of older adults in a specific setting; few facilities however offer assisted living services for low income populations (Heumann, 2004). Findings of the study can not be generalized to other samples and settings. There is no evidence to date that the model 
would replicate in a sample from a different population, such as among CCRC residents at other income levels, or community-dwelling older adults, or among a group of older adults with a mean age of 70 instead of 80 . Further research is necessary to determine if the effects of coping and transcendence generalize beyond the present study.

Standardized regression coefficients for proactive coping, and transcendence revealed that not only were both proactive coping $(\beta=.199, t=2.219, p=.029)$ and transcendence $(\beta=.523, t=5.659, p=.000)$ important contributors to successful aging, controlling for age, health, income, and function, but that transcendence was the greater contributor to the model, contributing about two and a half times more than proactive coping to the observed change in successful aging.

\section{Research Question 4}

The final research question asked about differences in adaptation, transcendence, and successful aging between participants who reported they were aging successfully and those who did not. This question could not be answered by the study due to the lack of variability in responses to the question, "Do you feel you are aging successfully?" Less than $5 \%$ of participants reported they were not aging successfully. Given similar findings in the feasibility study (McCarthy, 2009), this outcome was not unanticipated.

Strawbridge and his colleagues (2002) first observed that many older adults with chronic conditions and functional difficulties still rated themselves as aging successfully. The results of the present study were congruent with Strawbridge's study. Baltes and Carstensen's (1996) definition of successful aging. i.e., doing the best with what one has, may account for participant's positive views of success. Bias due to social desirability was also a possibility; participants might be more likely to state they were aging 
successfully, since success is a highly valued trait in Western culture (Holstein \& Minkler, 2003). As previously discussed, older adults' perceptions of their health, income level, and functional status were also more optimistic than suggested by objective measures and this might also account for an optimistic self-assessment of success in aging.

\section{Limitations of the Study}

The study had several limitations, and findings must be interpreted in light of these limitations. First, the study was cross-sectional, yielding only data at a single point in time. Especially in the older adult population, the passage of time and the accumulated changes associated with aging might be expected to create significant differences over

time. Additionally, it was possible participants' responses might vary based on mood, life events, or other external factors; these effects could not be assessed in the present study.

A second limitation, perhaps unavoidable in social science research, was a high probability of self-selection bias. This may have been reflected in the lack of variability in responses to the question on perceived successful aging. Despite random sampling, it is possible that older adults who were not satisfied with the way they were experiencing old age would be less likely to agree to participate. Thus results of the study may shed some light on older adults who were successfully aging, but very little was learned about older adults who were not aging successfully. A third limitation was the narrow target population selected for the study. It is possible that social and environmental conditions in the CCRC revealed a significant relationship which might not be identified in a different sample or setting. If the study was replicated among older adults living in their own homes, those with higher income levels, or older adults with more impaired 
functional levels, results might be different regardless of the strength of the effect size found for the regression model. Thus the findings of the study should not be generalized to other settings or populations.

Sample size was another limitation of the study. While significance was found for a model regressing successful aging on proactive coping and self-transcendence, with a sufficiently large coefficient of determination to suggest the model might replicate without undue shrinkage and be of practical significance, a larger sample might have revealed correlations or differences in the means between SAI and sociodemographic variables, as well as correlations between perceived and objective measures and differences in successful aging related to age, health, income, and function. Despite selfselection bias, a sufficiently large sample might have produced enough variability on responses to the question about perceived successful aging to provide useful information.

A final limitation was related to operationalization of the variables in the study. While a feasibility study (McCarthy, 2009) provided some information on the reliability and practical usefulness of the Proactive Coping Inventory, the Self-transcendence Scale, and the Successful Aging Inventory, none had been validated among older adults in a low-income CCRC, limiting evidence of internal validity. Cronbach's alpha coefficients did provide some support for the reliability of these instruments $(\mathrm{PCI}=.77, \mathrm{STS}=.73$, $\mathrm{SAI}=.82)$. However, concern for the validity of the instruments remains. This was particularly a concern for the Successful Aging Inventory, which was only recently developed by the theorist among community-dwelling older adults attending a senior citizen center, with quite different characteristics. While the results of the present study provide initial support for the SAI, the instrument has not previously been used in studies 
of older adults and test-retest statistics for the SAI were not obtained in the instrumentdevelopment study, thus evidence of validity was limited.

\section{Future Studies}

The overarching purpose of the present study was to explore the potential usefulness of the theory of successful aging (Flood, 2005) as a guide to a more holistic and inclusive perspective on successful aging. The results of the study support further investigation of the theory among other target populations and in other settings. Specifically, replication of the present study in a sample of middle- and upper-income residents in similar independent and assisted living communities would be of value, as would a study of successful aging among independent, community-dwelling older adults, contrasted with older adults receiving home health services or residing in a skilled nursing facility. Given the large response rate among African American participants in the present study, future studies should investigate the influence of race, minority status, and culture on how successful aging is perceived.

A qualitative study is needed to identify potential predictors of successful aging not detected in the study or found in the literature, which might account for the unexplained variance in the model. Specifically, optimism greater than expected based on objective measures was identified repeatedly in the present study. A tendency to be overly optimistic is one of the most common of all psychological errors (Myers, 2002), i.e., an inherent cognitive bias toward underestimating difficulty and overestimating ability (Kruger \& Burrus, 2004). Optimism has been linked to psychological and physical well-being, especially when coping with long-term, persistent, and uncontrollable life stressors (Robinson-Whelen, Kim, MacCallum, \& Kiecolt-Glaser, 1997), and has been 
found to change very little over the life course (Scheier \& Carver, 1993). Optimism and pessimism appear to be innate traits distinct from affectivity or external criteria and have been shown to correlate with well-being (Benyamini \& Roziner, 2008; Mroczek, Spiro, Aldwin, Ozer, \& Bossé, 1993).

Other potential factors which the literature suggests might be associated with successful aging include: resilience (Lee, Brown, Mitchell, \& Schiraldi, 2008), selfefficacy (Bandura, 1989; Lovelace, 1990; McAvay, Seeman, \& Rodin, 1996), psychological empowerment (Jones \& Rose, 2005; McCarthy, 2008; Richard, Gauvin, Gosselin \& Laforest, 2009), and a sense of control (Heckhausen \& Dweck, 1998; Wolinsky, Wyrwich, Babu, Kroenke, \& Tierney, 2003). Studies with larger samples are needed to identify additional predictors which might increase the variability explained by the model.

Further study is also needed to refine the Successful Aging Inventory, perhaps accounting for additional predictors of successful aging. It is possible that the present study, and other early studies based in Flood's (2005) theory may suggest other factors. The instrument must also be validated among a number of samples from the same population, and samples from slightly different populations. Further evidence of reliability and test-retest stability is also required.

Longitudinal studies are needed to assess changes in adaptation, transcendence, and successful aging over time. A repeated measures study is needed to gather data at several points in time, over a period of weeks or months. Replicating the present study among the same sample from the CCRC could explore changes in adaptation, transcendence and successful aging over time, such as the effects of increasing age, and 
decreasing health and functional status, while providing some indication of the stability of the instruments used to operationalize the present study.

Freund and Baltes (1998) suggested that successful aging declined with advancing age, due to decreased ability to adapt to accumulated losses. However, transcendence, according to both Tornstam (2005) and Reed (2003), increases with age. It is possible that a balance may exist between the two factors that would explain why increased age, decreased health, and more limited function were not significantly associated with successful aging. Further study to examine this possibility might be productive.

Also, while attrition is a common problem in longitudinal studies using samples with a mean age of 80 , a study of the young-old recruited at senior citizens centers, with a larger sample size, might allow sufficient retention to track the progress of a portion of the sample across the continuum of care over a number of years, from communitydwelling, to independent living, through assisted living, to nursing home placement. Certainly one major focus of the National Institute on Aging is to examine factors that influence the rate at which older adults experience decline and dependency (National Institute on Aging, 2009).

Finally, interventional studies designed to increase adaptation and/or transcendence are needed, not only to assess the practical usefulness of the theory, but more importantly because randomized controlled trials are required to establish causation. Potential interventions using both adaptation and transcendence might produce a synergistic effect, greatly increasing the effectiveness of interventions based on either one of the factors alone. A single example might be a physical activity program in which older adults learn a game or sports activity, then instruct and guide school age children in 
the activity. Older adults potentially might benefit from an increased sense of purpose in life, from passing on knowledge to the next generation, increased physical activity and awareness of health promotion. Learning new skills would provide cognitive stimulation, perhaps preventing or slowing the onset of dementia, while increasing self-efficacy or a sense of empowerment, and potentially, an expanded social network might increase resources for social, emotional, or functional support.

\section{Conclusion}

In summary, Flood (2005) offers a new definition of successful aging, merging both biomedical and psychosocial perspectives on aging, while adding the rarely studied perspective on successful aging of older adults themselves. Flood's theory of successful aging proposes that two factors, adaptation and transcendence, predict successful aging. The present study provides some preliminary evidence that both factors are associated with successful aging and potentially may have a positive effect on improving life satisfaction and quality of life among older adults, their families, and caregivers, while potentially decreasing the burden of the rapidly growing aging population on social, health, and economic systems.

The most widely used definition of successful aging (Rowe \& Kahn, 1997) excludes older adults with any degree of chronic disease or disability, focuses largely on behavioral determinants of health, and views aging from a biomedical perspective as an outcome defined by morbidity and mortality, rather than as a developmental process continuing across the lifespan. Rowe and Kahn (1998) stated the focus of their successful aging research was to identify the factors that put one older adult on skis and another in a wheelchair. However, disadvantages which might limit an older adult's potential to 
achieve success, such as multiple chronic disease, minority status or low income, are not taken into account (Holstein \& Minkler, 2003). Existential or spiritual aspects of successful aging also are not fully considered (Sadler \& Biggs, 2006) and use of normative, externally imposed criteria for success miss aspects of successful aging that older adults tell us add richness and meaning to life, even in the presence of disease and disability.

Flood's theory is multidimensional and includes the spiritual or existential domain not considered in other aging theories. The holistic mid-range theory is especially appropriate for nursing. While externally imposed definitions and normative criteria of existing successful aging theory result in excluding a large proportion of the older adult population from the potential for success, the new theory of successful aging is equally applicable to the older man on skis in Colorado, or the less advantaged elderly woman in a wheelchair.

The major finding of the dissertation study involves support for the significant contributions of both proactive coping and self-transcendence to successful aging. It is notable that $45.4 \%$ of the variance in successful aging was explained by these two factors, independent of age, income, function, or health. In particular, the role of transcendence has not previously been described. Given the contribution of selftranscendence to total successful aging, relative to proactive coping, this study has identified transcendence as an important new factor with the potential to increase successful aging, which has not previously been considered. The present study supports the conclusion that Flood's theory may merit further study of its potential to increase positive aging among older adults. 


\section{REFERENCES}

AARP Public Policy Institute. (2008). A balancing act: State long-term care reform. Public policy brief \# 161. Retrieved online on January 24, 2009 at http://assets.aarp.org/rgcenter/il/inb161_ltc.pdf

Abraham, J. D. \& Hansson, R. O. (1995). Successful aging at work: An applied study of selection, optimization, and compensation through impression management. Journals of Gerontology, Series B, 50(2), P94-103.

Adler, N. (1997). Purpose in life: Summary prepared in collaboration with the Psychosocial Working Group for the John D. and Catherine T. MacArthur Research Network on Socioeconomic Status and Health. Retrieved on October 10,2008 at http:/www.macses.ucsf.edu/Research/Psychosocial/notebook/purpose.html Allardt, E. (1993). Having, loving, being: An alternative to the Swedish model of welfare research. In M. C. Nussbaum \& A. K. Sen, (Ed.). The quality of life. New York: Oxford University Press.

American Heritage Dictionary of the English Language (4 ${ }^{\text {th }}$ ed.). (2003). Houghton Mifflin Company Online. Retrieved on January 4, 2009 at http://www.thefreedictionary.com/ageism 
Anderson, R. N. \& DeTurk, P. B. (2002). United States Life Tables, 1999. U.S. National Center for Health Statistics. 2002. National Vital Statistics Reports, Vol. 50, No. 6. Hyattsville, MD. Retrieved online on January 22, 2009 at http://www.cdc.gov/nchs/data/nvsr/nvsr50/nvsr50_06.pdf

Angel, J., \& Angel, R. (2006). Minority group status and healthful aging: Social structure still matters. American Journal of Public Health, 96(7), 1152-1159.

Aspinwall, L. G. \& Taylor, S. E. (1997). A stitch in time: Self-regulation and proactive coping. Psychological Bulletin, 121(3), 417-436.

Aspinwall, L. G. (2005). The psychology of future-oriented thinking: From achievement to proactive coping, adaptation, and aging. Motivation and Emotion, 29(4), 203235.

Atchley, R. C. (1989). A continuity theory of normal aging. The Gerontologist, 29, 183190.

Aud, M. A. \& Conn, V. (2003). A call for nursing research in assisted living facilities. Journal of Gerontological Nursing, 29(2), 3.

Austin, C. D. (1991). Aging well: What are the odds? Generations, 15(1), 73-75.

Baltes, B. \& Heydens-Gahir, A. (2003). Reduction of work-family conflict through use of selection, optimization, and compensation behaviors. Journal of Applied Psychology, 88(6), 1005-1018.

Baltes, M. M., \& Carstensen, L. L. (1996). The process of successful aging. Aging and Society, 16, 397-422.

Baltes, M. M., \& Carstensen, L. L. (2003). The process of successful aging: Selection, optimization, and compensation. In U. M. Staudinger \& U. E. R. Lindenberger 
(Eds.). Understanding human development: Dialogues with life span psychology. pp. 81-104.

Baltes, M. M., Wahl, H. \& Reichert, M. (1991). Successful aging in long-term care institutions. Annual Review of Gerontology and Geriatrics, 11, 311-337.

Baltes, P. B., \& Baltes, M. (1990). Psychological perspectives on successful aging: The model of selective optimization with compensation. In P. Baltes \& M. Baltes (Eds.), Successful Aging: Perspectives from the Behavioral Sciences. Cambridge, UK: Cambridge University Press, pp.1-34.

Baltes, P. B., Baltes, M. M., Freund, A. M., \& Lang, F. (1999). The measurement of selection, optimization, and compensation (SOC) by self report: Technical Report. Berlin: Max Plank Institute. Obtained through electronic communication with A. Freund on May 5, 2008.

Baltes, P. B. \& Smith, J. (2003). New frontiers in the future of aging: From successful aging of the young old to the dilemmas of the fourth age. Gerontology, Behavioral Science Section, 48, 123-135.

Bandura, A. (1977). Self-efficacy: Toward a unifying theory of behavior. Psychological Review, 84(2), 191-215.

Bandura, A. (1989). Regulation of cognitive processes through perceived self-efficacy. Developmental Psychology, 25, 729-735.

Bandura, A. (1997). Self-efficacy: The exercise of control. New York: Freeman. Bandura, A. (2004). Swimming against the mainstream: The early years from chilly tributary to transformative mainstream. Behavior Research and Therapy, 42, 613630. 
Barone, S. H., Roy, C. L., \& Frederickson, K. C. (2008). Instruments used in Roy Adaptation Model-based research: Review, critique, and future direction. Nursing Science Quarterly, 21(4), 353-362.

Bearon, L. B. (1996). Successful aging: What does the "good life" look like? North Carolina State University Forum for Family and Consumer Issues, 1(3), Retrieved online May 21, 2006 at http:/www.ces.ncsu.edu/depts/fcs/pub/aging.html.

Benyamini, Y. \& Roziner, I. (2008). The predictive validity of optimism and affectivity in a longitudinal study of older adults. Personality and individual Differences, 44(4), 853864.

Bode, C., de Ridder, D. T., Kuijer, R. G., \& Bensing, J. M. (2007). Effects of an intervention promoting proactive coping competencies in middle and late adulthood. The Gerontologist, $47(1), 42-51$.

Borawski, E. A., Kinney, J. M., \& Kahana, E., 1996;. The meaning of older adults' health appraisals: Congruence with health status and determinants of mortality. Journal of Gerontology: Social Sciences, 51B:S157-S170.

Borson, S., Scanlan, J., Brush, M., Vitaliano, P., \& Dokmak, A. (2000). The Mini-cog: A cognitive 'vital signs' measure for dementia screening in multi-lingual elderly. International Journal of Geriatric Psychiatry, 15(11), 1021-1027. Retrieved online On May 2, 2008 at http://geriatrics.uthscsa.edu/educational/med_students/minicog_admin.htm.

Bowling, A. (2007). Aspirations for older age in the 21st century: What is successful aging? International Journal of Aging \& Human Development, 64(3), 263-297. 
Bowling, A., \& Dieppe, P. (2005). What is successful aging and who should define it? British Medical Journal, 331, 1548-1551.

Bowling, A., Fleissig, A., \& Gabriel, Z. (2003). Let's ask them: A national survey of definitions of quality of life and its enhancements among people aged 65 and over. International Journal of Aging \& Human Development 56, 269-306.

Bowling, A., \& Iliffe, S. (2006). Which model of successful ageing should be used? Baseline findings from a British longitudinal survey of ageing. Age and Ageing, $35,607-614$.

Braam, A. W., Bramsen, I., van Tilburg, T. G., van der Ploeg, H. M., \& Deeg, D. (2006). Cosmic transcendence and framework of meaning in life: Patterns among older adults in The Netherlands. Journal of Gerontology, Series B, 61B(3), S121-128.

Braam, A. W., Deeg, D. J., van Tilburg, T. G., Beekman, A. T., \& van Tilburg, W. (1998). Gerotranscendence as a life cycle perspective: An initial empirical approach among the elderly in The Netherlands. [Abstract] Tijdschrift voor Gerontologie en Geriatrie, 29(1), 24-32.

Brody, J. E. (1996). Good habits outweigh genes as key to healthy old age. New York Times. February 28, C, 9:1. Retrieved September 5, 2007 at http:/query.nytimes.com/gst/fullpage.html?sec=health\&res=9D05E5D91139F93 BA15751C0A960958260

Butler, R.N. (1969). Age-ism: Another form of bigotry. The Gerontologist, 9, 243-246. Butler, R. N. \& Gleason, H. (Eds). (1985). Productive aging: Enhancing vitality in later life. New York: Springer. 
Cannuscio, C., Block, J., \& Kawachi, I. (2003). Social capital and successful aging: The role of senior housing. Annals of Internal Medicine, 139, 395-399.

Carstensen, L. L. \& Hartel (Eds.). (2006). When I'm 64. [Executive Summary]. Institutes of Medicine. Washington, D.C.: National Academy Press. Retrieved on October 14, 2008 at http://books.nap.edu/openbook.php?record_id=11474\&page=1

Carver, C. S. (1997). You want to measure coping but your protocol's too long: Consider the Brief COPE. International Journal of Behavioral Medicine, 4, 92-100.

Centers for Disease Control and Prevention. (n.d.). Chronic disease: Definition. National Center for Chronic Disease Prevention and Health Promotion Definition of chronic disease. Retrieved online on April 5, 2009 at www.healthgoods.com/education/health_information/General_Health/chronic _disease.htm.

Centers for Disease Control and Prevention and the Merck Company Foundation. (2008). The State of Aging and Health in America 2007. Whitehouse Station, NJ: The Merck Company Foundation. Retrieved online on October 12, 2008 at http://www.cdc.gov/aging/pdf/saha_2007.pdf.

Centers for Medicare and Medicaid website. (2007). Overview of long-term care: Types of long-term care. Retrieved online on March 4, 2009 at http://www.medicare.gov/LongTermCare/Static/TypesOverview.asp.

Cohen, J. (1988). Statistical power analysis for behavioral sciences ( $2^{\text {nd }}$ ed.). Mahwah, NJ: Lawrence Erlbaum Associates. 
Collins, S. R., Davis, K., Schoen, C., Doty, M. M., \& Kriss, J. L. (2006). Health coverage for aging baby boomers: Findings from the Commonwealth Fund Survey of Older Adults. Based on a presentation at the National Academy of Social Insurance $18^{\text {th }}$ Annual Conference, Older and Out of Work: Jobs and Social Insurance for a changing economy, Washington, D. C., January 20, 2006. Retrieved online on September 2, 2008 at http://www.commonwealthfund.org/ /media/Files/Publications/Fund\%20Report/ 2006/Jan/Health\%20Coverage\%20for\%20Aging\%20Baby\%20Boomers\%20\%20 Findings $\% 20$ from $\% 20$ The $\% 20$ Commonwealth $\% 20$ Fund $\% 20$ Survey $\% 20$ of $\% 2001$ der\%20Adults/884_Collins_hlt_coverage_aging_baby_boomers\%20pdf.pdf

Coward, D. (1990). The lived experience of self-transcendence in women with advanced breast cancer. Nursing Science Quarterly, 3, 162-169.

Coward, D. (1991). Self-transcendence and emotional well-being in women with advanced breast cancer. Oncology Nursing Forum, 18, 857-863.

Coward, D. (1995). The lived experience of self-transcendence in women with AIDS. Journal of Obstetric, Gynecologic, and Neonatal Nursing, 24, 314-318.

Coward, D. (1996). Self-transcendence and correlates in a healthy population. Nursing Research, 45, 116-122.

Crumbaugh, J. C. (1968). Cross-validation of Purpose in Life Test based on Frankl's concepts. Journal of Individual Psychology, 24, 74-81.

Crumbaugh, J. C. \& Maholick, L. T. (1964). An experimental study in existentialism: The psychometric approach to Frankl's concept of noogenic neurosis. Journal of Clinical Psychology, 20, 589-596. 
Crumbaugh, J., \& Maholick, L. (1969). Manual of instructions for the Purpose in Life Test. Munster, IN: Psychometric Affiliates.

Cumming, E., \& Henry, W. (1961). Growing old: the process of disengagement. New York: Basic Books.

Cutler, L. J. (2007). Physical environments of assisted living: Research needs and challenges. The Gerontologist, 47(Special issue III), 68-82.

Darwin, C. (1859). On the origin of species by means of natural selection, or the preservation of favoured races in the struggle for life. London: John Murray. Retrieved online on October 12, 2008 at http://www.literature.org/authors/darwincharles/the-origin-of-species/index.html

Decker, I. M. \& Reed, P. G. (2005). Developmental and contextual correlates of elders' anticipated end-of-life treatment decisions. Death Studies, 29(9), 827-846.

Depp, C. A., Glatt, S. J., \& Jeste, D. V. (2007). Recent advances in research on successful or healthy aging. Current Psychiatric Reports, 9, 7-13.

Depp, C. A., \& Jeste, D. V. (2006). Definitions and predictors of successful aging: A comprehensive review of larger quantitative studies. American Journal of Geriatric Psychology, 14, 6-20.

Derogatis, L. R., Lipman, R. S., Rickels, K., Uhlenhuth, E. H., \& Covi, L. (1974). The Hopkins Symptom Checklist (HSCL): A self report symptom inventory. Behavioral Science, 19, 1-15.

Duke, J., Leventhal, H., Brownlee, S. \& Brownlee, E. A. (2002). Giving up and replacing activities in response to illness. Journals of Gerontology: Psychological and Social Sciences, Series B, 57B(4), P367-376. 
Eldercare.gov (n.d.). Housing options for older adults: A guide for making housing decisions. National Association of Area Agencies on Aging, Administration on Aging, U.S. Department of Health and Human Services. Retrieved online on March 4, 2009 at http://www.eldercare.gov/Eldercare.NET/Public/Resource/ fact_sheets/pdfs?Housing\%200ptions\%20Booklet.pdf.

Erikson, E. H. (1963). Childhood and society (2nd ed.). New York: W. W. Norton.

Erikson, E. H. (1980). Identity and the life cycle ( $2^{\text {nd }}$ ed.). New York: Norton \& Norton Company, Inc.

Erikson, E. (1987). The life cycle completed. New York: Norton \& Norton Company, Inc.

Erikson, E. H. \& Erikson, J. M. (1997). The life cycle completed: Extended version with new chapters on the ninth stage of development. New York: W. W. Norton and Company, Inc.

Esteban-Santillan, C., Praditsuwan, R., Ueda, H., \& Geldmacher, D. S. (1998). Clock drawing test in very mild Alzheimer's disease. Journal of the American Geriatrics Society 46:1266-1269.

Fawcett, J. (2002). The nurse theorists: 21st century updates, Callista Roy. Nursing Science Quarterly, 15(4), 308-310.

Fentleman, D. L. (1990). Successful aging in a postretirement society; in M. M. Baltes \& Baltes, P. B. (Eds.). Successful aging: Perspectives from the behavioral sciences. Cambridge, UK: Cambridge University Press.

Ferraro, K. F. \& Shippee, T. P. (2009). Aging and cumulative in equality: How does in equality get under the skin? The Gerontologist, 49(3), 333-343. 
Fiksenbaum, L. M., Greenglass, E. R., \& Eaton, J. (2006). Perceived social support, hassles, and coping among the elderly. The Journal of Applied Gerontology, $25(1), 17-30$.

Fisher, B. J. \& Specht, D. (1992). Successful aging and life satisfaction: A pilot study for conceptual clarification. Journal of Aging Studies, 6(2), 191-202.

Fisher, B. J., \& Specht, D. (1995). Successful aging, life satisfaction, and generativity in later life. International Journal of Aging and Human Development, 41(3), 239251.

Fisher, B. J. \& Specht, D. (1999). Successful aging and creativity in later life. Journal of Aging Studies, 13, 457-472.

Flood, M. (2003). Successful aging: A concept analysis. Journal of Theory Construction and Testing, 6(2), 105-108.

Flood, M. (2005). A mid-range nursing theory of successful aging. Journal of Theory Construction \& Testing, 9(2), 35-39.

Flood, M. (2006). Exploring the relationships between creativity, depression, and successful aging. Activities, Adaptation \& Aging, 31(1), 55-71.

Flood, M. (2008). Successful Aging Inventory: Instrument development among community-dwelling Southern older adults. Manuscript in preparation, received from the author through electronic mail on June 16, 2008.

Folkman, S. (1997). Positive psychological states and coping with severe stress. Social Science and Medicine, 45(8), 1207-1221. 
Folstein, M., Folstein, S.E., McHugh, P.R. (1975). "Mini-Mental State" - a practical method for grading the cognitive state of patients for the clinician. Journal of Psychiatric Research, 12(3); 189-198.

Frederickson, K., Jackson, B. S., Strauman, T., \& Strauman, J. (1991). Testing hypotheses derived from the Roy Adaptation Model. Nursing Science Quarterly, 4(4), 168-174.

Freund, A. \& Baltes, P. (1998). Selection, optimization, and compensation as strategies for life management: Correlation with subjective indicators of successful aging. Psychology and Aging, 13(4), 531-543.

Freund, A. \& Baltes, P. B. (2000). The orchestration of selection, optimization, and compensation: An action-theoretical conceptualization of a theory of developmental regulation. In A. Flammer, W. J. Perrig, \& A. Grob (Eds.). Control of Human Behavior, Mental Processes, and Consciousness: Essays in Honor of the 60th Birthday of August Flammer. Philadelphia, PA: Lawrence Erlbaum Associates. pp. 35-58.

Freund, A. M. \& Baltes, P. B. (2002a). The adaptiveness of selection, optimization, and compensation as strategies of life management. The Journals of Gerontology Series B: Psychological Sciences and Social Sciences, 57, P426-P434.

Freund, A. M. \& Baltes, P. B. (2002b). Life-management strategies of selection, optimization, and compensation: Measurement by self-report and construct validity. Journal of Personality and Social Psychology, 82(4), 642-662.

Fried, L. P., Freeman, M., Endres, T. E., \& Wasik, B. (1997). Building communities that promote successful aging. Journal of Western Medicine, 167(4), 216-219.

Fry, P. S. (2003). Perceived self-efficacy domains as predictors of fear of the unknown and fear of dying among older adults. Psychology and Aging, 18(3), 474-486. 
Garfein, A. J., \& Herzog, A. R. (1995). Robust aging among the young-old, old-old, and oldest-old. Journal of Gerontology, 50B(2), S77-S87.

Gavan, C. (2003). Successful aging families: A challenge for nurses. Holistic Nursing Practice, 17(1), 11-18.

Gestdöttir, S. \& Lerner, R. M. (2007). Intentional self-regulation and positive youth development in early adolescence: Findings from the 4-H Study of Positive Youth Development. Developmental Psychology, 43(2), 508-521.

Gilhooly, M. (2007). Successful ageing in an area of deprivation. Public Health, 121(11), 814-821.

Glass, T. (2003). Assessing the success of successful aging. Annals of Internal Medicine, 139(5, Part 1), 382-383.

Gordon, P. (1998). The Paula Gordon show: Aging secrets. Series of six audio-recorded interviews with Dr. John Rowe on June 6. Recorded interview and text retrieved online on October 20, 2008 at http:/www.paulagordon.com/shows/rowe/

Greenglass, E. (2002). Proactive coping. In E. Frydenberg (Ed.), Beyond coping: Meeting goals, vision, and challenges. London: Oxford University Press, (pp. 37-62).

Greenglass, E., Fiksenbaum, L., \& Eaton, J. (2006). The relationship between coping, social support, functional disability, and depression in the elderly. Anxiety, Stress, and Coping, 19(1), 15-31.

Greenglass, E., Schwarzer, R., Jakubiec, D., Fiksenbaum, L., \& Taubert, S. (1999). The Proactive Coping Inventory (PCI): A multidimensional research instrument. [Technical report.] Retrieved online April 9, 2008 at http://www.psych.yorku.ca/greenglass/pci.php 
Greenglass, E. R., Schwarzer, R., \& Taubert, S. (1999). The Proactive Coping Inventory (PCI): A multidimensional research instrument. [Instrument.] Retrieved online on January 8, 2008 at http://www.psych.yorku.ca/greenglass/

Haas, B. K. (1999). A multidisciplinary concept analysis of quality of life. Western Journal of Nursing Research, 21(6), 728-742.

Hansen-Kyle, L. (2005). A concept analysis of healthy aging. Nursing Forum, 40(2), 45 57.

Harris-Kojetin, L., Joseph, A., Zimring, C., \& Keifer, K. (2005). Active living by design: Creating activity-enhancing residential settings. [Executive Summary]. Published online by the American Association of Homes and Services for the Aging.

Retrieved March 4, 2009 at http://www.aahsa.org/uploadedFiles/IFAS/Publications_amp;_Products/Active_ Living_by_design_Summary.pdf.

Havighurst, R. J. (1957). The leisure activities of the middle-aged. American Journal of Sociology, 63, 152-162.

Havighurst, R. J. (1961). Successful aging. The Gerontologist, 1, 8-13.

Havighurst, R. J. (1963). Successful aging. In R. H. Williams, C, Tibbits, \& W. Donahue. (Eds.). Processes of aging. New York: Atherton Press, pp. 299-320.

Havighurst, R. J., Neugarten, B. L., \& Tobin, S. S. (1963). Disengagement, personality and life satisfaction in the later years. In P. F. Hansen (Ed.). Age with a future. (pp. 419-425). Copenhagen: Munksgaard.

Hawes, C. \& Phillips, C. D. (2007). Defining quality in assisted living: Comparing apples, oranges, and broccoli. The Gerontologist, 47(Special issue III), 40-50. 
Healthy People 2010. (2000). United States Department of Health and Human Services, Centers for Disease Control and Prevention. Retrieved online on September 3, 2007 at http://www.healthypeople.gov/document/tableofcontents.htm\#under

Hebeler, H. J. (2007). Getting started in a financially secure retirement. Hoboken, NJ: John Wiley and Sons. Retrieved online on March 2, 2008 at http://www.amazon.com/Getting-Started-Financially-SecureRetirement/dp/0470117788

Heckhausen, J. \& Dweck, C. S. (1998). Motivation and self-regulation across the life span. New York: Cambridge University Press.

Heumann, L. F. (2004). Assisted living for lower income frail older persons from the housing and built environment perspective. Journal of Housing for the Elderly, 18(3), 165-178.

Hobbs, F. \& Stoops, N. (2002). Demographic trends in the $20^{\text {th }}$ century: Census 2000 special reports, Series CENSR-4. Washington, D. C.:U.S. Government Printing Office. Retrieved online on January 21, 2009 at http://www.jamesgoulding.com/Downloads/Census_Data_pdf/Population_Demog raphic_Trends_1900_2000_stats.pdf

Holstein, M. B., \& Minkler, M. (2003). Self, society, and the "new gerontology." The Gerontologist, 43(6), 787-796.

Houser, A., Fox-Grage, W., \& Gibson, M. J. (2009). Across the States 2009: Profiles of long-term care and independent living $\left(8^{\text {th }}\right.$ ed.). AARP Public Policy Institute. Retrieved online on March 7, 2009 at http://assets.aarp.org/rgcenter/il/d19105_2008_ats.pdf 
Idler, E. L. \& Benyamini, Y. (1997). Self-rated health and mortality: A review of twenty-seven community studies. Journal of Health and Social Behavior, 38(1), 21-37.

Johnson, J. K., Lie, L. Y., \& Yaffe, K. (2007). Executive function, more than global cognition, predicts functional decline and mortality in elderly women. The Journals of Gerontology Series A: Biological Sciences and Medical Sciences, 62, 1134-1141.

Jones, C. J. \& Rose, D. J. (2005). Physical activity instruction of older adults. Champaign, Illinois: Human Kinetics.

Jonnalagadda, S. \& Diwan, S. (2005). Health behaviors, chronic disease prevalence and self-rated health of older Asian immigrants in the U.S. Journal of Immigrant Health, $7(2), 75-83$.

Jönson, H. \& Magnusson, J. A. (2001). A new age of old age? Gerotranscendence and the re-enchantment of aging. Journal of Aging Studies, 15, 317-331.

Kahana, E. (n.d.) Brochure: Elderly Care Research Center, Department of Sociology. Case Western Reserve University at Cleveland, Ohio. Retrieved online on March 2, 2008 at http://www.case.edu/artsci/soci/ecrc/index.html

Kahana, E., Chirayath, H. T., Wisniewski, A. A., \& Kercher, K. C. (2003). How health adaptations ameliorate adverse effects of ill health on older adults' quality of life. Paper presented at the annual meeting of the American Sociological Association, Atlanta Hilton Hotel, Atlanta, GA. August 16.

Kahana, E. \& Kahana, A. (1996). Conceptual and empirical advances in understanding aging well through proactive adaptation. In Adulthood and aging: Research on continuities and discontinuities (pp. 18-40). 
Kahana, E., Kahana, B., \& Kelley-Moore, J. A. (2005). Coping with disability in late life: A longitudinal study of proactive adaptations. Paper presented at the annual meeting of the American Sociological Association, Marriott Hotel, Loews Philadelphia Hotel, Philadelphia, PA. August 12. Retrieved online on March 5, 2009 at http://www.allacademic.com/meta/p23372_index.htmlKahana, E.,

Kahana, E., Kahana, B., \& Kercher, K. (2003). Emerging lifestyles and proactive options for successful aging. Ageing International, 28(2), 155-176.

Kahana, E., Lawrence, R. H., Kahana, B., Kercher, K., Wisniewski, A., Stoller, E., et al. (2002). The long-term impact of preventive proactivity on quality of life of the old-old. Psychosomatic Medicine, 64(3), 382-394).

Kahn, R. L. (2002). On successful aging and well-being: Self-rated compared with Rowe and Kahn. The Gerontologist, 42, 725-726.

Kane, R. A. \& Wilson, K. B. (2007). Improving practice through research in and about assisted living: Implications for a research agenda. The Gerontologist, 47, 4-7.

Katz, R. V., et al. (2006). The Tuskegee Legacy Project: Willingness of minorities to participate in biomedical research. Journal of Health Care for the Poor and Underserved, 17(4), 698-715.

Keith, J., Fry, C. L., \& Ikels, C. (1990). Community as context for successful aging. In J. Sokolovsky, Ed. The cultural context of aging: World-wide perspectives. New York: Bergin and Garvey, pp. 245-261.

Kelley-Moore, J., Schumacher, J., Kahana, E., \& Kahana, B. (2006). When do older adults become "disabled"? Acquiring a disability identity in the process of health decline. Journal of Health \& Social Behavior,47. 126-141. 
Kentucky State Data Center. (2002). Census 2000 summary file income profile for Jefferson County: Household income by age of householder. Retrieved online on June 2, 2009 at http://ksdc.louisville.edu/sdc/census2000/58pageprofiles/JeffersonCounty3.pdf

Kirby, S. E., Coleman, P. G., \& Daley, D. (2004). Spirituality and well-being in frail and nonfrail older adults. Journal of Gerontology: Psychological Sciences, 59B(3), P123-P129.

Knickman, J., \& Snell, E. (2002). The 2030 Problem: Caring for aging baby boomers. Health Services Research, 37(4), 849-884. Retrieved online on April 15, 2007 at http://www.findarticles.com/p/articles/mi_m4149/is_4_37/ai_91568394.

Kressin, N. R., Meterko, M., \& Wilson, N. J. (2000). Racial disparities in biomedical research. Journal of the National Medical Association, 92(2), 62-69.

Kruger, J. \& Burrus, J. (2004). Egocentrism and focalism in unrealistic optimism and pessimism. Journal of Experimental Social Psychology, 40(3), 332-340.

Lang, F. R., Featherman, D. L., \& Nesselroade, J. R. (1997). Social self-efficacy and short-term variability in social relationships: The MacArthur Successful Aging Studies. Psychology and Aging, 12(4), 657-667.

Lang, F. R., Rieckmann, N., \& Baltes, M. M. (2002). Adapting to aging losses: Do resources facilitate strategies of selection, optimization, and compensation in everyday functioning? Journals of Gerontology: Psychological and Social Sciences, Series B, 57B(5), P501-509.

Lawton, M. P. (1975). The Philadelphia Geriatric Center Morale Scale: A revision. Journal of Gerontology, 30, 85-9. 
Lawton, M. P. (1991). A multidimensional view of quality of life in frail elders. New York: Academic Press.

Lawton, M. P. (2003). Lawton's PGC Morale Scale. Created by M. Powell Lawton (1923-2001) while at the Polisher Research Institute of the Philadelphia Geriatric Center (now known as the Abramson Center for Jewish Life). Retrieved November 8, 2007 from http://www.abramsoncenter.org/PRI/

Lee, H. S., Brown, S. L., Mitchell, M. M., \& Schiraldi, G. S. (2008). Correlates of resilience in the face of adversity for Korean women immigrating to the U.S. Journal of Immigrant and Minority Health, 10(5), 415-422.

Litwin, H. \& Sapir, E.V. (2009). Perceived income: Adequacy among older adults in 12 countries: Findings from the Survey of Health, Ageing, and Retirement in Europe. The Gerontologist, 49(3), 397-406.

Lovelace, E. A. (1990). Aging and cognition: mental processes, self-awareness, and interventions. Philadelphia: Elsevier Publishing.

Marek, K. D., Popejoy, L., Petroski, G., Mehr, D., Rantz, M., \& Lin, W. C. (2005). Clinical outcomes of aging in place. Nursing Research, 54(3), 202-211.

Marriner-Tomey, A. \& Alligood, M. R. (2006). Nursing theorists and their work. Philadelphia: Elsevier Health Sciences.

Maslow, A. (1943). A theory of human motivation. Psychological Review, 50, 370-396. Retrieved on December 8, 2008 at Classics in the History of Psychology at $\mathrm{C}: \backslash$ Documents and Settings $\backslash$ Valerie McCarthy $\backslash \mathrm{My}$

Documents $\backslash$ Dissertation $\backslash$ Variables - primary $\backslash$ Adaptation $1 \backslash$ Classics in the History of Psychology -- A_H_ Maslow (1943) A Theory of Human Motivation.mht 
Maslow, A. (1968). Toward a psychology of being, (2 ${ }^{\text {nd }}$ ed.). New York: Van Nostrand Reinhold.

Maslow, A. (1969). Various meanings of transcendence. Journal of Transpersonal Psychology, 1, 56-66.

Maslow, A. (1970). Motivation and Personality ( $2^{\text {nd }}$ ed.). New York: Harper.

Maslow, A. (1971). The Farther Reaches of Human Nature. New York: Viking Press.

Mauk, K. (2006). Gerontological nursing: Competencies for care. Sudbury, MA: Jones \& Bartlett Publishers.

McAvay, G. J., Seeman, T. E., \& Rodin, J. (1996). A longitudinal study of change in domain-specific self-efficacy among older adults. Journals of Gerontology Series B: Psychological Sciences and Social Sciences, 51(5), P243-P253.

McCarthy, V. L. (2008). A multidisciplinary concept analysis of empowerment: Implications for nursing. Journal of Theory Construction and Testing, 12(2), 6874.

McCarthy, V. (2009). A feasibility study to identify data collection and measurement instruments for a study exploring successful aging. Manuscript in preparation.

McCurry, S. M., Edland, S. D., Teri, L., Kukull, W. A., Bowen, J. D., McCormick, W. C., \& Larson, E. B. (1999). The cognitive abilities screening instrument (CASI): Data from a cohort of 2524 cognitively intact elderly. International Journal of Geriatric Psychiatry, 14(10), 882=888.

McEwen M. \& Wills, E. M. (2002). Theoretical basis for nursing. Philadelphia, PA: Lippincott, Williams, \& Wilkins. 
McFadden, S. (1996). Religion, spirituality, and older people. In J. Birren \& K. Schaie (Eds.), Handbook of the Psychology of Aging, ( $4^{\text {th }}$ ed.). London: Academic Press Ltd.

McInnis-Dittrich, K. (2005). Social work with elders: A biopsychosocial approach to assessment and intervention, ( $2^{\text {nd }}$ ed. $)$. Boston: Pearson Education, Inc.

McKee, P., \& Barber, E. (2001). Plato's theory of aging. Journal of Aging and Identity, $6(2), 93-104$.

Meleis, A. I. (2007). Theoretical nursing: Development and progress ( $3^{\text {rd }} \mathrm{ed}$.). Philadelphia, PA: Lippincott, Williams, \& Wilkins.

Moody, H. R. (2002). Conscious growth: A new level of growth in later life. Adapted from Conscious aging: A strategy for positive development in later life. In J. Ronch \& J. Goldfield (Eds). Mental wellness in aging: Strength-based approaches. Washington, D. C.: Human Services Press. Retrieved June 12, 2007 at http://www.hrmoody.com/art4.html

Moody, H. R. (2008). Review essay: Aging America and the boomer wars. The Gerontologist, 48(6), 839-843.

Mroczek, D. K., Spiro, A., Aldwin, C. M., \& Bossé, R. (1993). Construct validation of optimism and pessimism in older men: Findings from the normative aging study. Health Psychology, 12(5), 406-409.

Munro, B. H. (2005). Statistical methods for health care research (5 $5^{\text {th }}$ ed.). New York: Lippincott Williams \& Wilkins.

Myers, D. G. (2002). Intuition: Its powers and perils. New Haven, CT: Yale University Press. 
National Institute on Aging website. (2009). Research areas of the Division of Geriatrics and Clinical Gerontology. Retrieved online on May 15, 2009 at http://www.nia.nih.gov/ResearchInformation/ExtramuralPrograms/GeriatricsAnd ClinicalGerontology/

National Institute on Aging website. (n.d.). MacArthur Study of Successful Aging. Retrieved online on October 4, 2008 at http://www.nia.nih.gov/ResearchInformation/ScientificResources/StudyInfo.htm? id $=56$

National Institutes of Health. (2008). NIH summit: The science of eliminating health disparities. National Center on Minority Health and Health Disparities. Presented December 16-18. Retrieved online on March 2, 2009 at http://www.blsmeetings.net/2008healthdisparitiessummit/.

Nelson, A. (2008). Healthy communities for healthy aging: Reconstruction of America. Podium presentation to the American Public Health Association Annual Meeting in San Diego, CA on October 28.

Nelson, T. (2004). Ageism: Stereotyping and prejudice against older persons. Cambridge, MA: MIT Press.

Neugarten, B. L., Havighurst, R. J., \& Tobin, S. S. (1961). The measurement of life satisfaction. Journal of Gerontology, 16, 134-143.

Norusis, M. J. (2006). SPSS statistical procedures companion. Upper Saddle River, NJ: Prentice Hall. 
Ouwehand, C., de Ridder, D., \& Bensing, J. M. (2007). A review of successful aging models: Proposing proactive coping as an important additional strategy. Clinical Psychology Review, 27(8), 873-884.

Pearlin, L. (1999). Stress and mental health: A conceptual overview. In A. V. Horowitz \& T. L. Sheid (Eds.), Handbook for the study of mental health: Social contexts, theories and systems. Cambridge: Cambridge University Press.

Pearlin, L., Lieberman, M., Menaghan, E., \& Mullan, J. (1981). The stress process. Journal of Health and Social Behavior, 22, 337-56.

Pearlin, L. I. \& Schooler, C. (1978). The structure of coping. Journal of Health \& Social Behavior, 18, 2-21.

Peel, N. M., Bartlett, H. P., \& McClure, R. J. (2004). Healthy ageing: How is it defined and measured? Australian Journal on Ageing, 23(3), 115-119.

Peel, N. M., McClure, R. J., \& Bartlett, H. P. (2005). Behavioral determinants of health. American Journal of Preventive Medicine, 28(3), 298-304.

Phelan, E. A., Anderson, L. A., LaCroix, A. Z., \& Larson, E. B. (2004). Older adults' views of "successful aging": How do they compare with researchers' definitions? Journal of the American Geriatrics Society, 52, 211-216.

Phelan, E. A., \& Larson, E. B. (2002). Successful aging: Where next? Journal of the American Geriatrics Society, 50, 1306-1308.

Plato. (360 B.C.a). Republic, book I: Dialogue on Cephalus. (Trans. B. Jowett, 1888).

(Trans. B. Jowett, 1871). Published online by The Internet Classics Archive.

Retrieved online on September 10, 2007 at

http://classics.mit.edu/Plato/republic.2.i.html 
Plato. (360 B.C.b). Republic, book VII: The allegory of the cave. In S. Van Sell \& I. A. Kalofissudis. The evolving essence of the science of nursing: A complexity integration nursing theory. Published online in ICU Nursing Web Journal, 8, p. 135-140. Retrieved on September 10, 2007 at http://www.nursing.gr/Complexitytheory.pdf

Plato. (after 399 B.C.) The apology. (Trans. B. Jowett, 1888). (Trans. B. Jowett, 1871). Published online by The Internet Classics Archive. Retrieved online on September 10, 2007 at http://classics.mit.edu/Plato/republic.html.

Polit, D. E. \& Beck, C. T. (2003). Nursing research: principles and methods $\left(7^{\text {th }}\right.$ ed.). Philadelphia: Lippincott Williams \& Wilkins.

Poon, L. W., Gueldner, S. H., \& Sprouse, B. M. (2003). Successful aging and adaptation with chronic diseases. New York: Springer Publishing Company.

Power, M., Quinn, K., Schmidt, S., \& the WHOQOL-OLD Group. (2005). Development of the WHOQOL-OLD module. Quality of Life Research, 14, 2197-2214.

President's Council on Bioethics. (2002). Staff background paper: The promise and the challenge of aging research. Retrieved online on October 13, 2008 at http://www.bioethics.gov/background/agingresearch.html

Radloff, L. (1977). The CES-D scale: A self-report depression scale for research in the general population. Applied Psychological Measurement, 1, 385-401.

Rahman, M. O. \& Barsky, A. J. (2003). Self-reported health among older Bangladeshis: How good a health indicator is it? The Gerontologist, 43, 856-863.

Reed. P. G. (1986a). Religiousness among terminally ill and healthy adults. Research in Nursing and Health, 9, 35-42. 
Reed. P. G. (1986b). Developmental resources and depression in the elderly. Nursing Research, 35, 368-374.

Reed. P. G. (1987). Spirituality and well-being in terminally ill hospitalized adults. Research in Nursing and Health, 10, 335-344.

Reed. P. G. (1989a). Mental health of older adults. Western Journal of Nursing Research, $11,143-163$.

Reed. P. G. (1989b). Self-transcendence in aging scale: Mental health of older adults. Western Journal of Nursing Research, 11(2), 161-163.

Reed, P. G. (1991a). Toward a nursing theory of self-transcendence: Deductive reformulation using developmental theories. Advances in Nursing Science, 13(4), $64-77$.

Reed, P. G. (1991b). Self-transcendence and mental health in the oldest-old. Nursing Research, 40(1), 5-11.

Reed, P. G. (2003). The theory of self-transcendence. In P. R. M. J. Smith and Liehr (Ed.), Middle Range Theory for Nursing (pp. 145-165). New York: Springer Publications.

Reed, P. G., Shearer, N. C., \& Nicoll, L. H. (2004). Perspectives on Nursing Theory, (4 ${ }^{\text {th }}$ ed.). Philadelphia: Lippincott, Williams, and Wilkins.

Register, M. \& Herman, J. (2006). A middle range theory for generative quality of life for the elderly. Advances in Nursing Science, 29(4), 340-350.

Rice, V. H. (2000). Handbook of stress, coping, and health: implications for nursing research, theory, and practice. Thousand Oaks, CA: Sage Publications. 
Richard, L., Gauvin, L., Gosselin, C., \& Laforest, S. (2009). Staying connected:

Neighbourhood correlates of social participation among older adults living in an urban environment in Montreal, Quebec. Health Promotion International, 24(1), $46-57$.

Rimkus, A. \& Melinchock, M. D. (2005). Thesaurus of aging terminology. ( $8^{\text {th }}$ ed.). Washington, D.C.: Ageline database, AARP. Retrieved online on June 1, 2008 at http://assets.aarp.org/rgcenter/general/thesaurus.pdf.

Robinson-Whelen, S., Kim, C., MacCallum, R. C., \& Kiecolt-Glaser, J. K. (1997).

Distinguishing optimism from pessimism in older adults: Is it more important to be optimistic or not to be pessimistic? Journal of Personality and Social Psychology, 73(6), $1345-1353$.

Rogers, M. E. (1970). An introduction to the theoretical basis of nursing. Philadelphia, PA: F. A. Davis.

Rowe, J. W., \& Kahn, R. L. (1987). Human aging: Usual and successful. Science, 237, 143-149.

Rowe, J. W., \& Kahn, R. L. (1997). Successful aging. The Gerontologist, 37(4), 433-440.

Rowe, J., \& Kahn, R. (1998). Successful aging. New York: Random House.

Rowe, J. W., \& Kahn, R. L. (1999). The future of aging. Contemporary Longterm Care, 22(2), 36-44.

Roy, C. (1984). Introduction to nursing: An adaptation model ( $2^{\text {nd }}$ ed.). Englewood Cliffs, New Jersey: Prentice-Hall.

Roy, C. \& Andrews, H. A. (1999). Roy adaptation model ( $2^{\text {nd }}$ ed.). Stamford, CT: Appleton \& Lange. 
Runquits, J. J. \& Reed, P. G. (2007). Self-transcendence and well-being in homeless adults. Journal of Holistic Nursing, 25(1), 5-13.

Ryff, C. D. (1989). In the eye of the beholder: Views of psychological well-being among middle-aged and older adults. Psychology and Aging, 4(2), 195-210.

Sadler, E., \& Biggs, S. (2006). Exploring the links between spirituality and "successful aging." Journal of Social Work Practice, 20(3), 267-280.

Sadler, E., Braam, A., Broese van Groenou, M., Deeg, D., \& van der Geest, S. (2006). Cosmic transcendence, loneliness, and exchange of emotional support with adult children: A study among older parents in The Netherlands. European Journal of Aging, 3(3), 146-154.

Sarvimäki, A., \& Stenbock-Hult, B. (2000). Quality of life in old age described as a sense of well-being, meaning and value. Journal of Advanced Nursing, 32(4), $1025-1033$.

Saxena, S., Orley, J., \& the WHOQOL Group. (1997). Quality of life assessment: The World Health Organization perspective. European Psychiatry, 12(Suppl. 3), 263266.

Scheidt, R. J., Humphreys, D. R., \& Yorgason, J. B. (1999). Successful aging: What's not to like? Journal of Applied Gerontology, 18(3), 277-282.

Scheier, M. E, \& Carver, C. S. (1993). On the power of positive thinking: The benefits of being optimistic. Current Directions in Psychological Science, 2, 26-30.

Scherwitz, L., Pullman, M., McHenry, P., Gao, B., \& Ostaseski, F. (2006). A contemplative care approach to training and supporting Hospice volunteers: A 
prospective study of spiritual practice, well-being, and fear of death. Explore, 2(4), p. 304-313.

Schulz, R. \& Heckhausen, J. (1996). A life span model of successful aging. American Psychologist, 51(7), 702-714.

Schulz, A. \& Northridge, M. E. (2004). Social determinants of health: Implications for environmental health promotion. Health Education Behavior, 31(4), 455-471.

Schwarzer, R. (1998). General perceived self-efficacy in 14 cultures. Retrieved online on April 9, 2008 at http://userpage.fu-berlin.de/ health/lingua5.htm.

Schwarzer, R. (1999). The Proactive Attitude Scale (PA Scale). Retrieved online on April 9, 2008 at http://userpage.fu-berlin.de/ health/proactive.htm.

Schwarzer, R. \& Schwarzer, C. (1996). A critical survey of coping instruments. In M. Zeidner \& N. S. Endler (Ed.). Handbook of coping: Theory, research, and applications. New York: Wiley. pp. 107-132.

Schwarzer, R. \& Taubert, S. (2002). Tenacious goal pursuit and striving toward personal growth: Proactive coping. In E. Frydenberg (Ed.)., Beyond coping: Meeting goals, visions, and challenges. London: Oxford University Press, pp. 19-35.

Seeman, T. E., Unger, J. B., McAvay, G., \& Mendes de Leon, C. F. (1999). Self-efficacy beliefs and perceived declines in functional ability: MacArthur Studies of Successful Aging. The Journals of Gerontology, 54B(4), 214-222.

SeniorHousing.Net. (2008). Types of senior housing and care. Retrieved online on March 4, 2009 at http://www.seniorhousingnet.com/seniors/kyo/index.jhtml; \$sessionid\$1ZPILOBVVIDCOCQAQUBCFFQ. 
Shadish, W. R., Cook, T. D., \& Campbell, D. T. (2002). Experimental and quasiexperimental designs for generalized causal inference. Boston: Houghton Mifflin Company.

Shavers-Hornaday, V. L., Lynch, C. F., Burmeister, L. F., \& Torner, J. C. (1997). Why are African Americans underrepresented in medical research studies?: Impediments to participation. Ethnicity and Health, 2(1-2), 31-45.

Shu, B., Huang, C., \& Chen, B. (2003). Factors related to self-concept of elderly residing in a retirement center. Journal of Nursing Research, 11(1), 1-8.

Simons, C. M. (2002). Proactive coping, perceived self-efficacy, and locus of control as predictors of life satisfaction in young, middle-aged, and older adults.

Dissertation Abstracts International: Section B: The Sciences and Engineering, 63(3-B), pp. 1590.

Smit, B., Burton, I., Klein, R. J. T., \& Street, R. (1999). The science of adaptation: A framework for assessment. Mitigation and Adaptation Strategies for Global Change, 4(3-4), 199-213.

Sohl, S. J. (2008a). Refining the conceptualization of an important future-oriented selfregulatory behavior: Proactive coping [Abstract]. Retrieved online on June 2, 2008 at http://www.grad.sunysb.edu/ddd/pdf/SohlStephanie.pdf.

Sohl, S. J. (2008b). Refining the conceptualization of an important future-oriented selfregulatory behavior: Proactive coping. Unpublished dissertation. Electronic copy provided by author, September 28, 2008. 
Spitzer, W. J., Neuman, K., \& Holden, G. (2004). The coming of age of assisted living care: New options for senior housing and social work practice. Social Work in Health Care, 38(3), 21-45.

Stevens, J.P. (2002). Applied multivariate statistics for the social sciences (4th ed.) Mahwah, NJ: Lawrence Erlbaum Assoc., Inc.

Stevens-Ratchford, R. \& Diaz, T. (2003). Promoting successful aging through occupation: An examination of engagement in life. Activities, Adaptation, and Aging, 27(3/4), 19-37.

Stone, R. I. \& Reinhard, S. C. (2007). The place of assisted living in long-term care and related service systems. The Gerontologist, 47(Special issue III), 23-32.

Strawbridge, W. J., Cohen, R. D., Shema, S. J., \& Kaplan, G. A. (1996). Successful aging: Predictors and associated activities. American Journal of Epidemiology, $144,135-141$.

Strawbridge, W. J., Wallhagen, M., \& Cohen, R. (2002). Successful aging and wellbeing: Self-rated compared with Rowe and Kahn. The Gerontologist, 42, 727-733.

Thibault, J. M., Ellor, J.W. \& F. E. Netting. (1991) A conceptual framework for assessing the spiritual functioning and fulfillment of older adults in long term care settings. Journal of Religious Gerontology, 7 (4): 29-46.

Thomas, W. H. (2004). What are old people for?: How elders will save the world. Acton, MA: Vanderwyk \& Burnham.

Thomas, S. B. \& Quinn, S. C. (1991). The Tuskegee Syphilis Study, 1932 to 1972: Implications for HIV education and AIDS risk education programs in the black community. American Journal of Public Health, 81(11), 1498-1505. 
Tornstam, L. (1989). Gero-transcendence: A meta-theoretical reformulation of the disengagement theory. Aging: Clinical and Experimental Research, 1(1), 55-63.

Tornstam, L. (1994). Gero-transcendence: A theoretical and empirical exploration. In L.E. Tomas \& S.A. Eisenhandler (Ed.). Aging and the religious dimension, pp. 203-225. Westport, CT: Auburn House.

Tornstam, L. (1996). Gerotranscendence: A theory about meaning into old age. Journal of Aging and Identity, 1(1), 37-50.

Tornstam, L. (1997a). Gerotranscendence: The contemplative dimension of aging. Journal of Aging Studies, 11(2), 143-154.

Tornstam, L. (1997b). Gerotranscendence in a broad cross sectional perspective. Journal of Aging and Identity, 2(1), 17-36.

Tornstam, L. (1999). Late-life transcendence: A new developmental perspective on aging. In L. E. Thomas, \& S. A. Eisendandler (eds.), Religion, belief, and spirituality in late life, New York: Springer.

Tornstam, L. (2000). Transcendence in later life, Generations, Vol. XXIII, 4:10-14.

Tornstam, L. (2003). Gerotranscendence from young old age to old old age. Online publication from The Social Gerontology Group, Uppsala. URL: http://www.soc.uu.se/publications/fulltext/gtransoldold.pdf.

Tornstam, L. (2005). Gerotranscendence: A developmental theory. New York: Springer Publishing.

United Nations, Division for Social Policy and Development. (2000). 1999:

International Year of Older Persons. Retrieved online on April 13, 2007 at http://www.un.org/esa/socdev/iyop/iyopaag.htm 
Upchurch, S. (1999). Self-transcendence and activities of daily living: The woman with the pink slippers. Journal of Holistic Nursing, 17, 251-266.

U.S. Census Bureau. (2007). United States S0103. Population 65 years and over in the United States. American Community Survey: Three-year estimates. Retrieved online on March 7, 2009 at http://factfinder.census.gov/servlet/STTable?_bm=y\&qr_name=ACS_2007_3YR_G00_S0103\&-ds_name=ACS_2007_3YR_G00_\&_lang=en

U.S. Department of Health and Human Services (DHHS). (2009). Poverty guidelines updated periodically. Federal Register, 42 U.S.C. 9902(2). Retrieved online on March 10, 2009 at http://aspe.hhs.gov/POVERTY/09poverty.shtml

U.S. Department of Housing and Urban Development (HUD). (2008a). Income limits documentation system: Income limits summary for Jefferson County, Kentucky. Retrieved online on March 4, 2009 at http://www.huduser.org/datasets/il/il2008/2008summary.odn?INPUTNAME=ME TRO31140M31140*2111199999\%2BJefferson+County\&selection_type=county $\&$ stname $=$ Kentucky \&statefp $=21 \&$ year $=2008$.

U.S. Department of Housing and Urban Development (HUD). (2008b). Income limits documentation system: Income limits summary for Kentucky. Retrieved online on March 4, 2009 at http://www.huduser.org/ datasets/il/il2008/2008summary.odn? inputname $=$ STTLT $* 2199999999 \% 2$ BKentucky\&selection_type=county\&stname $=$ Kentucky\&statefp $=21 \&$ year $=2008$.

Vaillant, G. (2002) Aging well. Boston: Little, Brown and Co. 
Vaillant, G. E. \& Mukamal, K. (1990). Natural history of male psychological health: A 45-year study of predictors of successful aging at age 65. American Journal of Psychiatry, 147(1), 31-37.

Vaillant, G. \& Mukamal, K. (2001). Successful aging. The American Journal of Psychiatry, 158, $839-847$.

von Faber, M. A., Bootsma-van der Wiel, A., van Exel, E., Gussekloo, J., Lagaay, A., van Dongen, E., et al. (2001). Successful aging in the oldest-old: Who can be characterized as successfully aged? Archives of Internal Medicine, 161(22), 26942700. Retrieved on October 12, 2006 at http://archinte.amaassn.org/cgi/content/abstract/161/22/2694

Wadensten, B., \& Carlsson, M. (2003). Nursing theory views on how to support the process of ageing. Journal of Advanced Nursing, 42(2), 118-124.

Wadensten, B., \& Carlsson, M. (2005). Theory-driven guidelines for practical care of older people, based on the theory of gerotranscendence. Journal of Advanced Nursing, 4l(5), 462-470.

Wagner, P. (1976). The Roy Adaptation Model: Testing the Adaptation Model in practice. Nursing Outlook, 24(11), 682-685.

Walton, C. G., Shultz, C., Beck, C. M., \& Walls, R. (1991). Psychological correlates of loneliness in the older adult. Archives of Psychiatric Nursing, 5(3), 165-170.

Wang, C. \& Satariano, W. A. (2007). Self-rated current and future health independently predict subsequent mortality in an aging population. Journals of Gerontology, Series A: Biological Sciences and Medical Sciences, 62, 1428-1434. 
Whitbourne, S. K. \& Willis, S. L. (2006). The baby boomers grow up: Contemporary perspectives on midlife. New York: Routledge. Retrieved online on January 23, 2009 at http://books.google.com/books?id=EXWXSiNnZ10C

WHOQOL-BREF Group. (1998). The development of the World Health Organization WHOQOL-BREF quality of life assessment. Psychological Medicine, 28, 551558.

Wilson, K. B. (2007). Historical evolution of assisted living in the United States, 1979 to the present. The Gerontologist, 47(Special issue III), 8-22.

Wolinsky, F. D., Wyrwich, K. W., \& Babu, A. N. (2003). Age, aging, and the sense of control among older adults: A longitudinal reconsideration. Journal of Gerontology: Social Sciences, 58B(4), S212-S220.

Wong, P. T. (1998). Spirituality, meaning, and successful aging. In the human quest for meaning. P. T. Wong \& P. Fry (Eds.). Mahwah, New Jersey: Lawrence Erlbaum Associated.

Woodford, H. J. \& George, J. (2007). Cognitive assessment in the elderly: A review of clinical methods. QJM: An international Journal of Medicine, 100(8), 469-484.

World Health Organization (WHO). (1946). Definition of health. In the Preamble to the Constitution of the World Health Organization. Official Records of the World Health Organization, No. 2, p. 100. Retrieved on October 13, 2008 at http://www.who.int/about/definition/en/print.html

World Health Organization, Regional Office of the West Pacific. (1999). Press release: Active and healthy aging. World Health Day, 07 April 1999. Retrieved online on 
April 13, 2007 at

http://www.wpro.who.int/media_centre/press_releases/pr_19990407.htm

Young, L. M., Baltes, B. B., \& Pratt, A. K. (2007). Using selection, optimization, and compensation to reduce job/family stressors: Effective when it matters. Journal of Business and Psychology, 21(4), 511-539.

Zimmerman, S. M., Phelps, E., \& Lerner, R. M. (2007). Intentional self-regulation in early adolescence: Assessing the structure of selection, optimization, and compensation processes. European Journal of Developmental Science, 1(2), 271298.

Zimmerman, S. \& Sloane, P. D. (2007). Definition and classification of assisted living. The Gerontologist, 47(Special issue III), 33-39. 


\title{
Appendix A
}

Fig. 2. Mini-Cog Dementia Test Instructions and Scoring

\section{The Mini-Cog Dementia Test}

\begin{abstract}
ADMINISTRATION
The test is administered as follows:

1. Instruct the patient to listen carefully to and remember 3 unrelated words and then to repeat the words. 2. Instruct the patient to draw the face of a clock, either on a blank sheet of paper or on a sheet with the clock circle already drawn on the page. After the patient puts the numbers on the clock face, ask him or her to draw the hands of the clock to read a specific time.

3. Ask the patient to repeat the 3 previously stated words.

\section{SCORING}

Give 1 point for each recalled word after the CDT distractor.

Patients recalling none of the three words are classified as demented (Score $=0$ ).

Patients recalling all three words are classified as non-demented (Score $=3$ )

Patients with intermediate word recall of 1-2 words are classified based on the CDT (Abnormal = demented;

Normal $=$ non-demented)
\end{abstract}

Note: The CDT is considered normal if all numbers are present in the correct sequence and position, and the hands readably display the requested time.

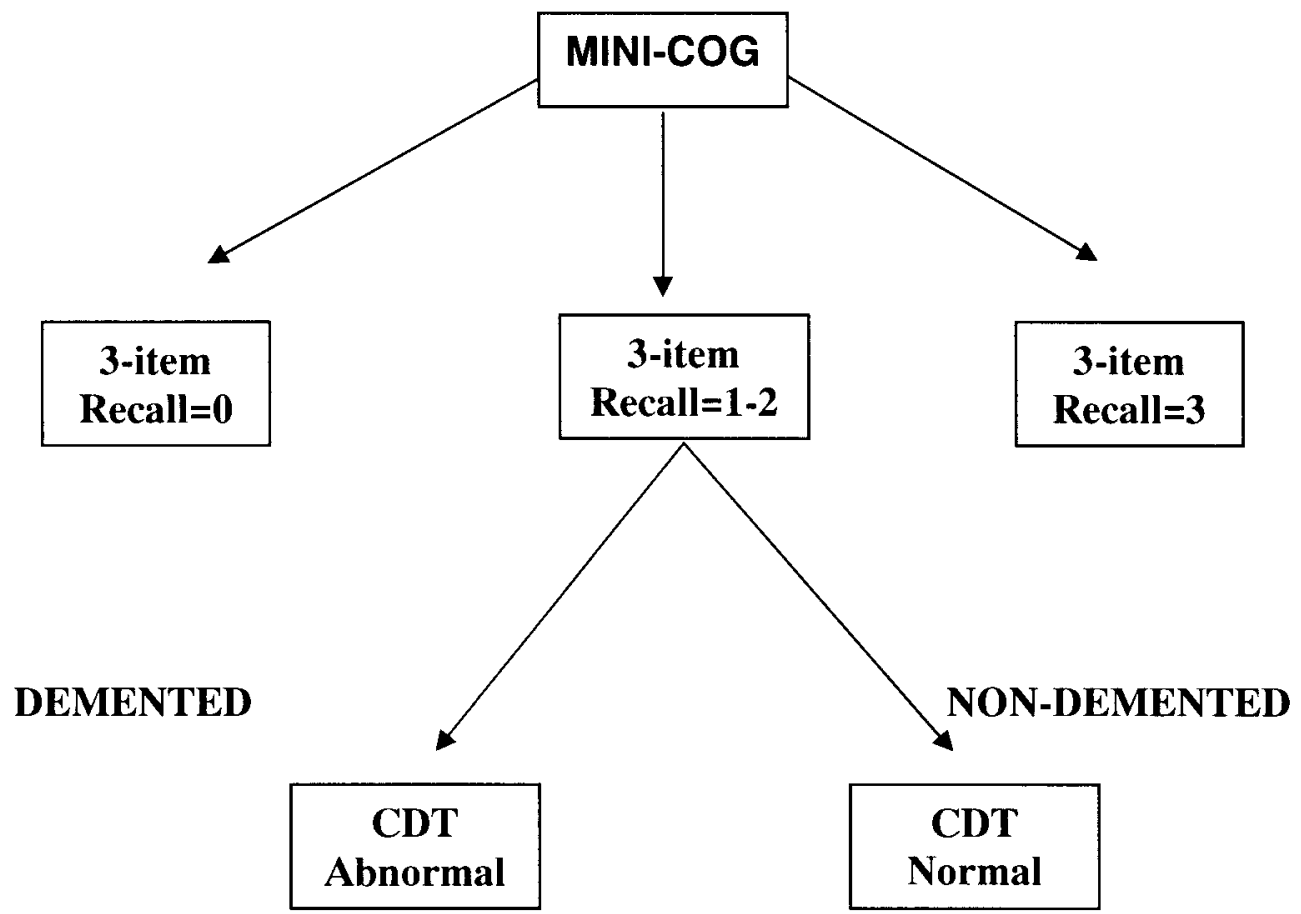

Source: Borson, Scanlan, Brush, Vitallano, \& Dokmak. 2000, p. 1024.

Page 1 of 2 
Appendix A, continued

Mini-Cog Assessment Test for Dementia

Mini-Cog Assessment

\section{CLOCK DRAW TEST}

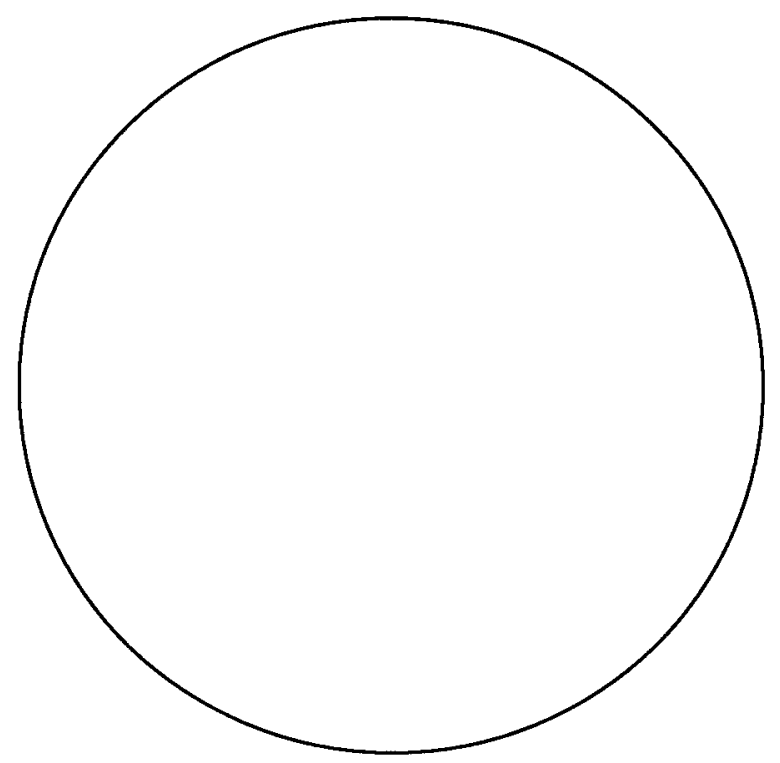

1) Inside the circle, please draw the hours of a clock as they normally appear.

2) Place the hands of the clock to represent the time: "ten minutes after eleven o'clock."

\section{Three Recall Words:}

Source: Borson, Scanlan, Brush, Vitallano, \& Dokmak (2000).

Permission is hereby granted to reproduce, post, download, and/or distribute, this material in its entirety only for not-for-profit educational purposes, provided that The Hartford Institute for Geriatric Nursing, College of Nursing, New York University is cited as the source. This material may be downloaded and/or distributed in electronic format, including PDA format. Available on the internet at www.hartfordign.org and/or www.ConsultGeriRN.org. E-mail notification of usage to: hartford.ign@anvu.edu

Page 2 of 2 
Appendix B

Recruitment Flyer

\section{What's It Like To Grow Old?}

If you can spare about 1 hour out of your day, we would like to ask you to participate in a research study on how people over 65 see life and adjust to the changes and challenges of aging. Participants will meet with a registered nurse, who is a PhD candidate in the School of Nursing at the University of Louisville, to fill out a set of four questionnaires.

We will telephone some residents of Chapel House and Friendship House, selected at random, to ask you to agree to answer questions such as, "Do you share your wisdom and experience with others?" and "Do you manage to do the things you need to do to take care of your home and yourself?" If we phone you, you'll be free to say yes and participate in the study, or say no, and not participate - it's entirely up to you.

If you agree to participate, we will schedule a day and time that is convenient for you. Small groups of five to seven people will meet at Chapel House or Friendship House. You will be asked to mark down answers to questions without talking them over with each other. The questions will be printed in large type and will be read out loud by the nurse. It should take less than an hour and no one else at the meeting will see your answers.

A small group discussion will follow, to share your thoughts and ask any questions you may have. Participants will receive a $\$ 5$ gift certificate to the market on the ground floor of Chapel House as compensation for your time.

Participation is free and strictly voluntary. You may change your mind or drop out of the study at any time before you finish completing the survey. You will not be paid to participate. You may benefit by knowing that your accumulated life wisdom might help other older adults in future - and it may be enjoyable in the process. 
Questions may be addressed to:

Barbara J. Speck, PhD, RN Associate Professor

University of Louisville School of Nursing

Louisville, KY 40292

(502) $852-8513$ or

bjspec01@gwise.louisville.edu or Valerie McCarthy, RN, BSN

$\mathrm{PhD}$ Candidate, Graduate Fellow

University of Louisville School of Nursing

Louisville, KY 40292

(502) $819-5037$ or

vemcca01@gwise.louisville.edu

Please consider sharing your thoughts on growing older. The results of the study may be used in future to help plan health and social policies or programs and to provide aging services targeted to what you - the experts on aging - tell us you want and need. 
Appendix C

Schedule of Data Collection Sessions (Confidential)

Data collection sessions: Monday through Friday, $10 \mathrm{am}, 2 \mathrm{pm}, 4 \mathrm{pm}$, and $6 \mathrm{pm}$.

\begin{tabular}{|l|l|l|l|l|l|}
\hline $\begin{array}{c}\mathbf{N}= \\
\mathbf{1 3 5}\end{array}$ & Name & Telephone \# & $\begin{array}{c}\text { Apartment } \\
\#\end{array}$ & Date & Time \\
\hline & & & & & \\
\hline & & & & & \\
\hline & & & & & \\
\hline & & & & & \\
\hline & & & & & \\
\hline & & & & & \\
\hline & & & & & \\
\hline & & & & & \\
\hline
\end{tabular}

$$
\text { Page }
$$

of 
Appendix D

Reminder of scheduled data collection session

\section{REMINDER}

Thank you so much for agreeing to participate in the research study.

Please plan on attending a 45 to 60 minute session to fill out the questionnaire at:

Date:

Time:

Place:

You'll notice we've also included two sets of forms for you to look at.

YOU DON'T NEED TO DO ANYTHING WITH

EITHER ONE RIGHT NOW.

We thought you might like to have them to look over in advance and to keep for your records. The first is a Consent Form, which explains your rights. The second is a form called HIPAA, about how we will keep your private information confidential. We'll go over both forms together at our meeting and I will be glad to answer any questions at that time, or you may phone me if you have questions you'd like answered before we meet.

If you cannot attend at the scheduled time, please phone me at 502-819-XXX.

Don't forget you will receive a $\$ 5$ gift card for the market on the ground floor of Chapel House as compensation for your

Reminders will be placed in sealed envelopes, along with copies of the consent and HIPAA forms, and then placed in the individual boxes at each participant's apartment door. 
Appendix E

Subject Informed Consent Document

IRB: 09.0209

\section{Subject Informed Consent Document}

Exploring a New Theory of Successful Aging for Usefulness among Low-Income

Older Adults in an Independent and Assisted Living Retirement Community

\section{Introduction and Background Information}

You are invited to participate in a research study. The study is being conducted by Barbara J. Speck, PhD, RN and Valerie L. McCarthy, BSN, RN, PhD candidate. The study is sponsored by the University of Louisville School of Nursing and will be conducted at the Christian Care Community. Approximately 160 residents over the age of 65 will be invited to spend about one hour filling out four questionnaires.

\section{Purpose}

The purpose of this study is to understand the ways people adjust as they get older. We want to know how their outlook on life may change with age and how that might change what really matters most to them. We want to find out what older adults think about successful aging so we can plan policies, programs, and services for older adults.

\section{Procedures}

In this study, about 135 residents of Chapel House and Friendship House at the Christian Care Community will be chosen at random, like by flipping a coin, to be invited to answer questions on their experiences and feelings about getting older. We will also ask participants to take a three minute test to show they were able to understand and answer our questions. Groups of residents will at Christian Care Community to complete questionnaires. Participants will complete four questionnaires in small groups of five to seven residents. Participants will mark their own answers on the questionnaires, so other participants will not know each other's answers. Participants will be free to decline to answer any particular questions that make them uncomfortable. A single session of about one hour will be needed to answer all the questions.

\section{Potential Risks}

There are few risks associated with completing the questionnaires. There could be embarrassment in answering personal questions. Also, it is possible someone might feel upset remembering things from their life or thinking about getting older. We will ask the Christian Care Community staff to comfort or support anyone who needs help. There may also be unforeseen risks. 


\section{Benefits}

The possible benefits of this study include a chance to share your opinions and ideas. The information collected may not benefit you directly but the information learned in this study may be helpful to others.

\section{Compensation}

Participants who attend a session and fill out questionnaires will receive a $\$ 5$ gift card for the market in Chapel House to compensate you for your time.

\section{Confidentiality}

Total privacy cannot be guaranteed. Your privacy will be protected to the extent permitted by law. If the results from this study are published, your name will not be made public. While unlikely, the following may look at the study records:

The University of Louisville Institutional Review Board, Human Subjects

Protection Program Office, and Privacy Office.

Office for Human Research Protections (OHRP),

Office of Civil Rights

You will mark all answers to questions on paper questionnaires. These records will be stored in a locked file cabinet in a secure area at the School of Nursing. Answers will also be kept in a password protected computer.

\section{HIPAA Research Authorization}

The Health Insurance Portability and Accountability Act of 1996 (HIPAA) provides federal safeguards for protected health information (PHI). Examples of $\mathrm{PHI}$ are your name, address, and age if 89 or older. $\mathrm{PHI}$ may also include the number of chronic health conditions you tell us you have and how you rate your own health. Your PHI may not be used or shared without your agreement, unless it meets one of the HIPAA exceptions. If you agree to take part in this research you may be required to sign a "Research Authorization" form. This allows the use and sharing of your PHI by those listed in the "Research Authorization.

\section{Voluntary Participation}

Taking part in this study is voluntary. You may choose not to take part at all. If you decide to be in this study you may stop taking part at any time. If you decide not to be in this study or if you stop taking part at any time, you will not lose any benefits for which you may qualify. 


\section{Research Subject's Rights, Questions, Concerns, and Complaints}

If you have any concerns or complaints about the study or the study staff, you have three options:

You may contact the principal investigator by phone at (502) $852-X X X$ or by e-mail at bjspec01@gwise.louisville.edu.

If you have any questions about your rights as a study subject, questions, concerns or complaints, you may call the Human Subjects Protection Program Office (HSPPO) (502) 852-5188. You may discuss any questions about your rights as a subject, in secret, with a member of the Institutional Review Board (IRB) or the HSPPO staff. The IRB is an independent committee composed of members of the University community, staff of the institutions, as well as lay members of the community not connected with these institutions. The IRB has reviewed this study.

If you want to speak to a person outside the University, you may call 1 877-852-1167. You will be given the chance to talk about any questions, concerns or complaints in secret. This is a 24 hour hot line answered by people who do not work at the University of Louisville.

This paper tells you what will happen during the study if you choose to take part. Your signature means that this study has been discussed with you, that your questions have been answered, and that you will take part in the study. This informed consent document is not a contract. You are not giving up any legal rights by signing this informed consent document. You will be given a signed copy of this paper to keep for your records.

Signature of Subject/Legal Representative

Date Signed

Signature of Person Explaining the Consent Form (Co-Investigator)

Date Signed

Signature of Primary Investigator

Date Signed

LIST OF INVESTIGATORS

PHONE NUMBERS

Barbara J. Speck, PhD, RN

(502) 852-XXX or bjspec01@gwise.louisville.edu

Valerie McCarthy, $\mathrm{PhDc}, \mathrm{RN}$

(502) 819-XXX or vemcca01@gwise.louisville.edu 
Appendix F

\section{RESEARCH AUTHORIZATION USE AND DISCLOSURE OF YOUR HEALTH INFORMATION FOR RESEARCH}

Research Authorization form for participants aged 89 years and above.

Principal Investigator: Speck, Barbara

IRB\# : 09.0209

Title of the Research Study: Exploring a New Theory of Successful Aging for Usefulness among Low-Income Older Adults in an Independent and Assisted Living Retirement Community

\section{What information about you may be used or given out in the research study?}

HIPPA Statement: Information that identifies you and might relate to your health or medical condition may be used or given out in this study. Information that identifies you can include your name, address, telephone number, birth date, Social Security number or other details about you. In the current study the only information that identifies you that will be collected is your age in years. Information that relates to your mental health or medical condition includes the results of the Mini-Cog test to screen for dementia, the number of chronic medical conditions you report to us, and your rating of your own health (very good, good, fair, poor). Additional information that may be obtained from subjects that is not covered by the activities and procedures listed in this authorization, such as information about financial and social circumstances, or educational level will also be obtained.

\section{From who or where can we get information about you?}

Some people may see your mental or physical health information and may share this information during this study. These include the primary investigator and co-investigator as well as authorized medical, nursing, or social service staff of the Christian Care Community.

\section{Who may see your health information?}

Other organizations may see your health information during the study in order to conduct the study and to make sure it is being done as it should. These include: Governmental entities that have the right to see or review your health information, such as the Office of Human Research Protections and the Food and Drug Administration. The Institutional Review Board (IRB), including Human Subjects Protections Program Office (HSPPO), and Health Care providers, as appropriate, for your medical treatment at Christian Care Community, and people who are responsible for research and HIPAA oversight at the University of Louisville, and any other outside entity with whom health information will be shared.

Page 1 of 5 
4. Why will this information be used and given out?

Your information will be used and given out to conduct the study and to evaluate the results of the study. Your information may also be used to meet the reporting requirements of governmental agencies. Your information will be used to seek immediate assistance for you from Christian Care Community nursing or social services staff should you become emotionally distressed, confused, or ill during administration of the survey questionnaire.

5. Is your health information protected after it has been given to others?

If your health information is given to someone not covered by these policies and laws, that information may no longer be protected, and may be used or given out without your permission. In many cases the study sponsor, the clinical research organizations and other entities with whom the sponsor contracts are not covered by these policies and laws.

6. What if you decide not to give your permission to use and give out your health information?

You do not have to give your permission to use or give out your health information. However, if you do not give permission, you may not participate in the study.

7. May you withdraw or cancel your permission?

You may cancel your agreement to allow your health information to be used or given out at any time by completing a written Revocation Form and forwarding it to the Human Subjects Protections Program Office, MedCenter One, Suite 200, 501 E. Broadway, Louisville, KY 40202.

A revocation form may be obtained from your investigator or the Human Subjects Protections Program Office website at http://research.louisville.edu/UHSC. If you have any questions, call Human Subjects Protections Program Office at 852-5188. If you revoke your authorization, you are leaving the study. If you leave the study, no new health information about you will be gathered after that date. However, information gathered before that date may be used or given out if it is needed for the study or any follow-up for the study.

A participant may change his/her mind at any time, up to the completion of the data collection process, and may drop out of the study without penalty. If a participant chooses to drop out of the study prior to completion of the data collection process, partially completed surveys will be destroyed in the presence of the participant.

Page 2 of 5 


\section{Does this authorization have an end date?}

The time period when information can be used or disclosed under this authorization ends when all activities related to this study are completed.

\section{Have you given up any legal rights by signing this form?}

By signing this authorization form, you have not waived any of the legal rights you would have were you not in a research study.

FOR ADULTS (OR MINORS) CAPABLE OF GIVING AUTHORIZATION:

Subject's Signature

Date Signed

Printed Name

FOR CHILDREN OR ADULTS NOT CAPABLE OF GIVING AUTHORIZATION:

Signature of Parent/Surrogate/Guardian/Health Care Agent for Subject Date Signed

Printed Name

Relationship of Surrogate to Subject:

NOTE: THE PRINCIPAL INVESTIGATOR MUST:

- PROVIDE A COPY OF THE SIGNED AUTHORIZATION TO THE SUBJECT

- RETAIN THE ORIGINAL SIGNED AUTHORIZATION IN THE RESEARCH RECORD

- PLACE A COPY OF THE SIGNED AUTHORIZATION IN THE SUBJECT'S MEDICAL RECORD 


\section{REVOCATION OF RESEARCH AUTHORIZATION}

Principal Investigator: Speck, Barbara

IRB\#: Exempt

Title of the Research Study: Exploring a New Theory of Successful

Aging for Usefulness among Low-Income Older Adults in an

Independent and Assisted Living Retirement Community

Return To:

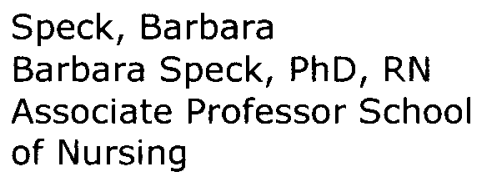

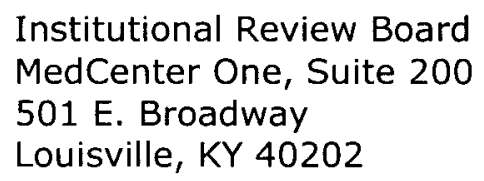

To Whom It May Concern:

I would like to discontinue my participation in the research study noted above. I understand that health information already collected will continue to be used as discussed in the Authorization I signed when joining the study.

At this point, in addition to ending participation, I would like to (choose one):

\section{$\square$ Withdraw from Study and Revoke Authorization:}

Revoke my authorization for the future use and disclosure of protected health information.

In some instances, the research team may need to use your information even after you revoke your authorization, for example, to notify you or government agencies of any health or safety concerns.

\section{$\square$ Withdraw from Study but Continue Authorization:}

Revoke my authorization for the future use and disclosure of protected health information.

In some instances, the research team may need to use your information even after you revoke your authorization, for example, to notify you or government agencies of any health or safety concerns. 
I understand that I will receive confirmation of this notice.

$\overline{\text { Signature of Subject }} \quad$ Date Signed $\quad$ Print Name

Birthdate Address Phone Number

\section{Optional:}

I am ending my participation in this study because:

Page 5 of 5 
Appendix G

Proactive Coping Inventory

\section{Reactions to Daily Events Questionnaire}

Instructions to Subjects: "The following statements deal with reactions you may have to various situations. Indicate how true each of these statements is depending on how you feel about the situation. Do this by checking the most appropriate box."

\begin{tabular}{|c|c|c|c|c|}
\hline $\begin{array}{l}\text { The Proactive } \\
\text { Coping Scale }\end{array}$ & $\begin{array}{l}\text { Not at } \\
\text { all true }\end{array}$ & $\begin{array}{c}\text { Barely } \\
\text { true }\end{array}$ & $\begin{array}{c}\text { Somewhat } \\
\text { true }\end{array}$ & $\begin{array}{c}\text { Completely } \\
\text { true }\end{array}$ \\
\hline $\begin{array}{l}\text { 1. I am a "take } \\
\text { charge" person. }\end{array}$ & 1 & 2 & 3 & 4 \\
\hline $\begin{array}{l}\text { 2. I try to let things } \\
\text { work out on } \\
\text { their own. }\end{array}$ & 1 & 2 & 3 & 4 \\
\hline $\begin{array}{l}\text { 3. After attaining a } \\
\text { goal, I look for } \\
\text { another, more } \\
\text { challenging one. }\end{array}$ & 1 & 2 & 3 & 4 \\
\hline $\begin{array}{l}\text { 4. I like challenges } \\
\text { and beating the } \\
\text { odds. }\end{array}$ & 1 & 2 & 3 & 4 \\
\hline $\begin{array}{l}\text { 5. I visualize my } \\
\text { dreams and try to } \\
\text { achieve them. }\end{array}$ & 1 & 2 & 3 & 4 \\
\hline $\begin{array}{l}\text { 6. Despite numerous } \\
\text { setbacks, I usually } \\
\text { succeed in getting } \\
\text { what I want. }\end{array}$ & 1 & 2 & 3 & 4 \\
\hline
\end{tabular}

Page 1 of 3 


\begin{tabular}{|c|c|c|c|c|}
\hline $\begin{array}{l}\text { The Proactive } \\
\text { Coping Scale }\end{array}$ & $\begin{array}{l}\text { Not at } \\
\text { all true }\end{array}$ & $\begin{array}{l}\text { Barely } \\
\text { true }\end{array}$ & $\begin{array}{c}\text { Some- } \\
\text { what } \\
\text { true }\end{array}$ & $\begin{array}{c}\text { Completely } \\
\text { true }\end{array}$ \\
\hline $\begin{array}{l}\text { 8. I always try to find } \\
\text { a way to work } \\
\text { around obstacles; } \\
\text { nothing really } \\
\text { stops me. }\end{array}$ & 1 & 2 & 3 & 4 \\
\hline $\begin{array}{l}\text { 9. I often see myself } \\
\text { failing so I don't } \\
\text { get my hopes up } \\
\text { too high. }\end{array}$ & 1 & 2 & 3 & 4 \\
\hline $\begin{array}{l}\text { 10. When I apply for a } \\
\text { position, I imagine } \\
\text { myself filling it. }\end{array}$ & 1 & 2 & 3 & 4 \\
\hline $\begin{array}{l}\text { 11. I turn obstacles } \\
\text { into positive } \\
\text { experiences. }\end{array}$ & 1 & 2 & 3 & 4 \\
\hline $\begin{array}{l}\text { 12. If someone tells } \\
\text { me I can't do } \\
\text { something, you } \\
\text { can be sure I will } \\
\text { do it. }\end{array}$ & 1 & 2 & 3 & 4 \\
\hline $\begin{array}{l}\text { 13. When I } \\
\text { experience a } \\
\text { problem, I take the } \\
\text { initiative in } \\
\text { resolving it. }\end{array}$ & 1 & 2 & 3 & 4 \\
\hline $\begin{array}{l}\text { 14. When I have a } \\
\text { problem, I usually } \\
\text { see myself in a no- } \\
\text { win situation. }\end{array}$ & 1 & 2 & 3 & 4 \\
\hline
\end{tabular}

Greenglass, E. (2002). Chapter 3. Proactive coping. In E. Frydenberg (Ed.), Beyond coping: Meeting goals, vision, and challenges. London: Oxford University Press, (pp. 37-62).

Page 2 of 3 


\section{Title of Proactive Coping Scale Given to Respondents: Reactions to Daily Events Questionnaire}

\section{Administration:}

"The following statements deal with reactions you may have to various situations. Indicate how true each of these statements is depending on how you feel about the situation. Do this by checking the most appropriate box."

Respondents are presented with four alternatives: "not at all true", "barely true", "somewhat true", "completely true."

\section{Scoring:}

In scoring responses, 1 is assigned to "not at all true", 2 to "barely true", 3 to "somewhat true" and 4 to "completely true". Please note that the following 3 items of the Proactive Coping subscale are reverse scored: "I try to let things work out on their own", "I often see myself failing so I don't get my hopes up too high", and "When I have a problem, I usually see myself in a no-win situation". That is, a score of 1 should be recoded to a score of 4 , a score of 2 should be recoded to a score of 3 , a score of 3 should be recoded to a score of 2 , and a score of 4 should be recoded to a score of 1 . Responses should be added to obtain a summed score for each of the 7 subscales. The range of scores for the proactive coping subscale is $14-56$. There are no cut-off scores, as we do not endorse the view that people should be categorized this way.

Greenglass, E. R., Schwarzer, R., \& Taubert, S. (1999). The Proactive Coping Inventory ( $P C l)$ : A multidimensional research instrument. [On-line publication] at http://www.psych.yorku.ca/greenglass/ In public domain.

REVERSE CODE ITEMS 2, 9, and 14 
Appendix $\mathrm{H}$

Self-transcendence Scale with Instructions for Administration, and Scoring

\section{Self-Transcendence Scale}

DIRECTIONS: Please indicate the extent to which each item below describes you. There are no right or wrong answers. I am interested in your frank opinion.

As you respond to each item, think of how you see yourself at this time in your life. Circle the number that is the best response for you.

$\begin{array}{llll}\text { Not } & \text { Very } & \text { Some- } & \text { Very } \\ \text { at all } & \text { little } & \text { what } & \text { much }\end{array}$

At this time of my life, I see myself as:

1. Having hobbies or interests I enjoy.

1

2

3

4

2. Accepting myself as I grow older...

1

2

3

4

3. Being involved with other people or

1

2

3

4

my community when possible.

4. Adjusting well to my present life situation........

1

2

3

4 
Not

at all

5. Adjusting to changes in my

physical abilities...

6. Sharing my wisdom or experience with others.

7. Finding meaning in my past experiences.

8. Helping others in some way

9. Having an ongoing interest in learning

10. Able to move beyond some things that once seemed so important.

11. Accepting death as part of life

12. Finding meaning in my spiritual beliefs.
Very

little

Some-

what

Very

much

4

4

4

4

4 


$\begin{array}{rlll}\text { Not } & \text { Very } & \text { Some- } & \text { Very } \\ \text { at all } & \text { little } & \text { what } & \text { much }\end{array}$

13. Letting others help me when I may ................. 1 need it.

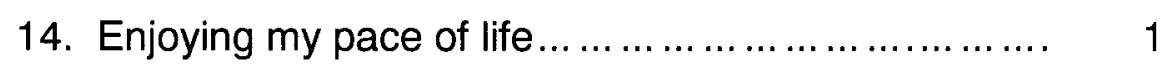

2

3

4

15. Dwelling on my past losses

1

2

2 much

Thank you very much for completing these questions. Please write down any additional comments that may help us understand your views. 


\section{STS Scale Instructions for Administration and Scoring:}

DIRECTIONS: Please indicate the extent to which each item below describes you. There are no right or wrong answers. I am interested in your frank opinion. As you respond to each item, think of how you see yourself at this time of your life. Circle the number that is the best response for you.

The STS is a one-dimensional scale, consisting of 15 items that together identify characteristics of a matured view of life that expand boundaries of the self. The STS is administered as an interview to elicit perceptions of the degree to which the respondents experience each item in their current life. It may also be administered as a questionnaire.

Responses are based upon a 4-point scale ranging from 1 for "not at all" to 4 for "very much." The final score reflects overall level of self-transcendence and takes into account intraindividual differences in self-transcendence experiences; a low score on one item may be offset by a high score on another item

(Reed, electronic correspondence on January 11, 2007.

\section{SCORING THE STS: REVERSE CODE ITEM 15}

The possible scores for each item range from 1 to 4 , with:

Not at all $=1$ to Very much $=4$, as indicated on the questionnaire.

NOTE that the scoring on item 15 is reversed such that:

Not at all $=4$ to Very much $=1$.

Note: Minor modifications in wording of some items were made in January 2007, to improve its clarity, based upon

feedback from researchers and respondents. The changes are minor and do not substantively change the instrument.

To calculate the STS Score:

- The respondent's scores for items 1 through 15 are added together.

- Then divide this score by the number of items answered (which should be 15, but may be less if there is missing data).

- The STS Score possible range is 1.0 to 4.0 .

- In previous research, the STS Score may have been calculated based upon the sum only, in which case the possible range reported was 15 to 60 . Dividing this sum by 15 results in a range of 1.0 to 4.0 .

(C)Pamela Reed, PhD, RN, FAAN 1987 Page 4 of $4 \quad$ Used with permission. 
Appendix I

Successful Aging Inventory

\section{Successful Aging Inventory}

Read each statement carefully. Circle the answer that matches how you feel right now.

Remember there are no right or wrong answers.

\begin{tabular}{|l|c|c|c|c|c|}
\hline & $\begin{array}{c}\text { Hardly } \\
\text { Ever }\end{array}$ & $\begin{array}{c}\text { Some of } \\
\text { the time }\end{array}$ & $\begin{array}{c}\text { About } \\
\text { half the } \\
\text { time }\end{array}$ & $\begin{array}{c}\text { Most of } \\
\text { the time }\end{array}$ & $\begin{array}{c}\text { Almost } \\
\text { always }\end{array}$ \\
\hline $\begin{array}{l}\text { 1. I manage to do the things that I need } \\
\text { to do to take care of my home and to } \\
\text { take care of myself (eating, bathing, } \\
\text { dressing). }\end{array}$ & 0 & 1 & 2 & 3 & 4 \\
\hline $\begin{array}{l}\text { 2. I have been able to cope with the } \\
\text { changes that have occurred to my } \\
\text { body as I have aged. }\end{array}$ & 0 & 1 & 2 & 3 & 4 \\
\hline $\begin{array}{l}\text { 3. I look forward to the future. } \\
\text { 4. I feel able to deal with my own } \\
\text { aging. }\end{array}$ & 0 & 1 & 2 & 3 & 4 \\
\hline 5. I feel able to cope with life events. & 0 & 1 & 2 & 3 & 4 \\
\hline
\end{tabular}

Page 1 of 4 
Successful Aging Inventory, continued

\begin{tabular}{|c|c|c|c|c|c|}
\hline & $\begin{array}{l}\text { Hardly } \\
\text { Ever }\end{array}$ & $\begin{array}{l}\text { Some of } \\
\text { the time }\end{array}$ & $\begin{array}{c}\text { About } \\
\text { half the } \\
\text { time }\end{array}$ & $\begin{array}{l}\text { Most of } \\
\text { the time }\end{array}$ & $\begin{array}{l}\text { Almost } \\
\text { always }\end{array}$ \\
\hline $\begin{array}{l}\text { 6. I can come up with solutions to } \\
\text { problems. }\end{array}$ & 0 & 1 & 2 & 3 & 4 \\
\hline $\begin{array}{l}\text { 7. I am good at thinking of new ways to } \\
\text { solve problems. }\end{array}$ & 0 & 1 & 2 & 3 & 4 \\
\hline $\begin{array}{l}\text { 8. I enjoy doing creative new things or } \\
\text { making things. }\end{array}$ & 0 & 1 & 2 & 3 & 4 \\
\hline 9. I am in a positive, pleasant mood. & 0 & 1 & 2 & 3 & 4 \\
\hline $\begin{array}{l}\text { 10. I think of my loved ones who have } \\
\text { passed away and feel close to them. }\end{array}$ & 0 & 1 & 2 & 3 & 4 \\
\hline $\begin{array}{l}\text { 11. I spend time in prayer or doing some } \\
\text { kind of religious activity. }\end{array}$ & 0 & 1 & 2 & 3 & 4 \\
\hline
\end{tabular}


Successful Aging Inventory, continued

\begin{tabular}{|c|c|c|c|c|c|}
\hline & $\begin{array}{l}\text { Hardly } \\
\text { Ever }\end{array}$ & $\begin{array}{l}\text { Some of } \\
\text { the time }\end{array}$ & $\begin{array}{l}\text { About } \\
\text { half the } \\
\text { time }\end{array}$ & $\begin{array}{l}\text { Most of } \\
\text { the time }\end{array}$ & $\begin{array}{l}\text { Almost } \\
\text { always }\end{array}$ \\
\hline $\begin{array}{l}\text { 12. As I have aged, the way I think of the } \\
\text { world has changed. }\end{array}$ & 0 & 1 & 2 & 3 & 4 \\
\hline $\begin{array}{l}\text { 13. I would rather have a few close friends } \\
\text { than many casual ones. }\end{array}$ & 0 & 1 & 2 & 3 & 4 \\
\hline $\begin{array}{l}\text { 14. Sometimes there can be two right } \\
\text { answers to a problem or situation. }\end{array}$ & 0 & 1 & 2 & 3 & 4 \\
\hline $\begin{array}{l}\text { 15. A relationship with God or some higher } \\
\text { power is important to me. }\end{array}$ & 0 & 1 & 2 & 3 & 4 \\
\hline $\begin{array}{l}\text { 16. I feel interest in/concern for the next } \\
\text { generation }\end{array}$ & 0 & 1 & 2 & 3 & 4 \\
\hline 17. My life is meaningful. & 0 & 1 & 2 & 3 & 4 \\
\hline
\end{tabular}


Successful Aging Inventory, continued

\begin{tabular}{|l|c|c|c|c|c|}
\hline & $\begin{array}{c}\text { Hardly } \\
\text { Ever }\end{array}$ & $\begin{array}{c}\text { Some of } \\
\text { the time }\end{array}$ & $\begin{array}{c}\text { About } \\
\text { half the } \\
\text { time }\end{array}$ & $\begin{array}{c}\text { Most of } \\
\text { the time }\end{array}$ & $\begin{array}{c}\text { Almost } \\
\text { always }\end{array}$ \\
\hline $\begin{array}{l}\text { 18. I am overall satisfied with my life right } \\
\text { now. }\end{array}$ & 0 & 1 & 2 & 3 & 4 \\
\hline $\begin{array}{l}\text { 19. I feel that I serve a purpose in this } \\
\text { world. }\end{array}$ & 0 & 1 & 2 & 3 & 4 \\
\hline $\begin{array}{l}\text { 20. Being the age that I am now is as good } \\
\text { or better that I thought it would be. }\end{array}$ & 0 & 1 & 2 & 3 & 4 \\
\hline
\end{tabular}

(Flood, 2008)

Page 4 of 4 
Appendix J

Investigator-developed Questionnaire

CIRCLE THE NUMBER next to the answer below that most nearly tells us about you. Circle only one answer to each question. If you are not sure, circle the number next to the answer that is closest to correct for you.

Gender: 1. Female 2. Male Age in years:

Race: 1. African American 2. White 3. Other

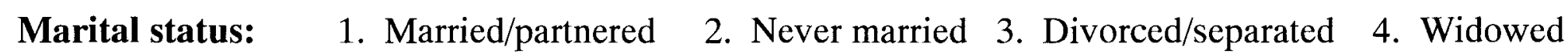

Education level: 1 . Less than high school diploma 2. High school graduate

3. Some college

4. College graduate or higher

Income: $\quad 1$. Barely enough to get by 2 . Enough to get by 3 . Enough to live in some luxury

How would you describe your health? $\quad \begin{array}{llll}\text { 1. Very good } & \text { 2. Good } & 3 \text {. Fair } & 4 \text {. Poor }\end{array}$

\section{Page 1 of 2}


Chronic health conditions: Please circle all of the chronic health conditions listed below that you have been told by a doctor that you have:

1. Hypertension/high blood pressure

2. Arthritis

3. Heart disease

4. Diabetes

5. Lasting effects of a stroke

6. Respiratory disease such as chronic bronchitis/asthma/emphysema

7. Cancer (not currently receiving treatment)

8. Other chronic disease

\section{Functional ability:}

1. Able to live independently, without help from another person more than occasionally

2. Need assisted living services such as help with bathing, medications, or getting around
Do you feel you are aging successfully?
Circle only one:
1. Yes
2. No 
Appendix K

Standardized Protocol for Administration of Survey Instruments

1. Physical accommodations: Data collection will be conducted in small groups of 5 to 7 participants in a private meeting space with a door that will be closed during the data collection session. Sufficient space will be provided, with tables and chairs, so that participants are not seated in near each other, to minimize the potential for interaction. Only participants randomly selected and scheduled will be allowed in the data collection session. CCRC staff will be immediately available to relocate any resident with dementia who proves distracting and is unable to complete the Mini-Cog test, while the investigator remains focused on the group.

2. Introduction to be read at beginning of each session: "We are very interested in learning how you decide which things in life are important to you, how you go about accomplishing what you want in life, and how you feel about things that matter to you. What we learn may help us understand growing old from the perspective of the "experts" - you! We hope that what we learn will help us to provide ways to help elders stay as active, healthy, and independent as possible for as long as possible.

"That's why I am asking you to take your time and be very thoughtful in how you respond to the questions, even though answering many questions can get boring sometimes. But we need to know the answer to the very last question just as much as the first question. Please do not talk among yourselves until all questions are completed. We want to know what each of you thinks, without anyone else influencing you. There are no "right" or "wrong" answers. We will have a chance to talk as a group afterward if you would like. Don't "help" anyone!

"Please open the manila envelope in front of you and take out the packet of questionnaires and the laminated card inside - but don't write on anything yet. Please look at the sample question on the first page and notice you have several choices for your response. You may also look at the same choices in large print on the laminated card in your envelope. If your response falls somewhere between the choices on your questionnaire, please mark the choice that is nearest to your response, but try not to leave any questions blank, if possible - unless you feel uncomfortable with the question for any reason; then feel free to leave it blank and go on to the next question.

"I will read each of the questions aloud, repeating it twice, but I can read it as many times as you'd like, just let me know. You can follow along on your questionnaire packet but I also want anyone who doesn't see well to get to LISTEN to the questions too. Let me know if I speak too softly. I'd like you to mark only one answer for each question in your questionnaire packet. If there isn't any answer that is exactly right, please pick the answer closest to what you think. Mark the answer you choose on your copy of the survey for each question. There are four sets of questions and it should take about 45 to 60 minutes to finish.

Page 1 of 4 
"If you need me to repeat a question, I will be glad to, but what I can't do is to explain the question, change the wording, or talk with you about more than how to mark your answer. That's so anything I say doesn't change what someone might feel or think was the "right" answer.

"But when we get done with the questionnaire, I look forward to getting to talk as much as you'd like about how you feel or things the questions made you think about. I also want to ask you to wait until the study is over before you talk with other residents about the questions asked. This is so each person in the study starts off from the same place and doesn't accidentally give responses based on what they have heard instead of how they really feel."

3. Consent \& HIPAA: Copies of both forms were previously delivered to participants' apartments, along with appointment reminders, to allow time for consideration before attending data collection session or being asked to sign Consent and HIPAA form. These copies will be retained by participants for their records. In the data collection sessions, participants will be told to turn to the first page in the packet, the Consent. The Consent will be read aloud and explained, and the participants will have an opportunity to ask questions or decline to participate before signing. The same process will be repeated for the HIPPA form.

4. Disruption or Distraction: dementia-type behaviors demonstrated by either scheduled participants or other residents of the community will be managed by retirement community staff and/or a research assistant who will remain present until it is apparent that all participants are able to cooperate with survey procedures. Staff or assistant will relocate any unscheduled resident or participant who is disruptive or distracting, according to CCRC policies and procedures.

5. Mini-Cog Test: will be administered according to instructions on the form. Residents will be told, "Now, we will begin with a little memory test. Don't worry about 'passing' or 'failing.' It's just to have a record, showing you were able to understand the process and answer questions." Participants will be allowed to complete the questionnaires regardless of the Mini-Cog score, however, responses for participants with abnormal Mini-Cog scores will not be entered into the computer database, nor will they count toward the total number of participants.

6. Sensory Limitations: Communication problems or sensory limitations such as vision or hearing loss will be addressed by speaking slowly, distinctly, and in a low pitch, and loudly enough to be heard by respondents, even those with some hearing loss. Participants' attention will be directed to the enlarged versions of each scale, which be explained prior to beginning each survey instrument. Each item will be read aloud at least twice. If a participant does not understand the question, the item will be re-read, making an effort to communicate clearly. Items will not be explained or elaborated upon. The only admissible clarification is an exact repetition of the item as it appears on the scale or to direct a respondent to the sample answer scale.

Page 2 of 4 
7. Survey Questionnaire Packets will be provided in manila envelopes. The questionnaire packets will be stapled and will include:
a. Consent \& HIPAA form*
b. Mini-Cog test
c. Self-transcendence scale (STS)
d. Proactive coping inventory (PCI)
e. Successful aging inventory (SAI)
f. Investigator-developed questionnaire
g. Laminated card with examples of Likert scales in large print
h. Blank receipt for gift card

* Copies were previously delivered to participants' apartments, along with appointment reminders, to allow time for consideration before attending data collection session or being asked to sign Consent and HIPAA form. These copies will be retained by participants for their records. These copies will be retained by participants for their records. Additional copies will also be offered at data collection.

8. Gift cards will be distributed at the end of data collection, and the participants will be asked to sign and date receipts included as last page in survey questionnaire packets. Participants will be reminded to NOT discuss the study or specific questions with other residents. Participants will be asked to place the entire questionnaire packet back in the blank manila envelope and hand it to the investigator. Participants will be thanked as they leave.

9. Confidentiality: When each data collection session has been completed, survey questionnaire packets, in blank envelopes to ensure privacy, are received by the investigator.

After participants have cleared the room, the investigator will process the packets to maintain confidentiality, using the following protocol:

a. Mini-Cog Results: Mini-Cog tests will be scored and the results entered in the space provided on the investigator- administered questionnaires.

b. Unique identifiers: Unique identifiers will be assigned for each participant and entered in the space provided on the investigator-administered questionnaire. Identifiers will distinguish between normal Mini-Cog scores (01-1 to 01-135) and abnormal Mini-Cog scores (02-1, 02-2, 02-3, ...).

c. Study Rosters: The unique identifier and participant's name, obtained from the Consent, will also be entered on study rosters (one for included data, i.e., normal Mini-Cog scores and one for excluded data, i.e., abnormal Mini-Cog scores). Study rosters will be kept in the investigator's immediate possession or in a locked storage cabinet in a secure area of the school of nursing, separate from survey questionnaires.

Page 3 of 4 
d. Consent and HIPAA forms: Consent and HIPAA forms will be separated from survey packets, after names are recorded on the study rosters, and will be kept in the investigator's immediate possession or in a locked storage cabinet in a secure area of the school of nursing, separate from survey questionnaires.

e. Survey Questionnaires: The survey questionnaires will be filed in alphabetical order in one of two file boxes or folders (one for included data, i.e., normal Mini-Cog scores and one for excluded data, i.e., abnormal Mini-Cog scores). The boxes or folders will be kept in the investigator's immediate possession or in a locked storage cabinet in a secure area of the school of nursing, separate from identifying information.

f. The Director of Social Services will be notified of abnormal results of Mini-Cog tests, as well as any dementia-type behavior or emotional distress observed by the investigator, so that appropriate actions may be taken, according to CCRC policies, to ensure the participant's safety and welfare.

Page 4 of 4 
Appendix L

Laminated examples of Likert scales printed in 24 point font and laminated

Self-Transcendence Scale (STS)

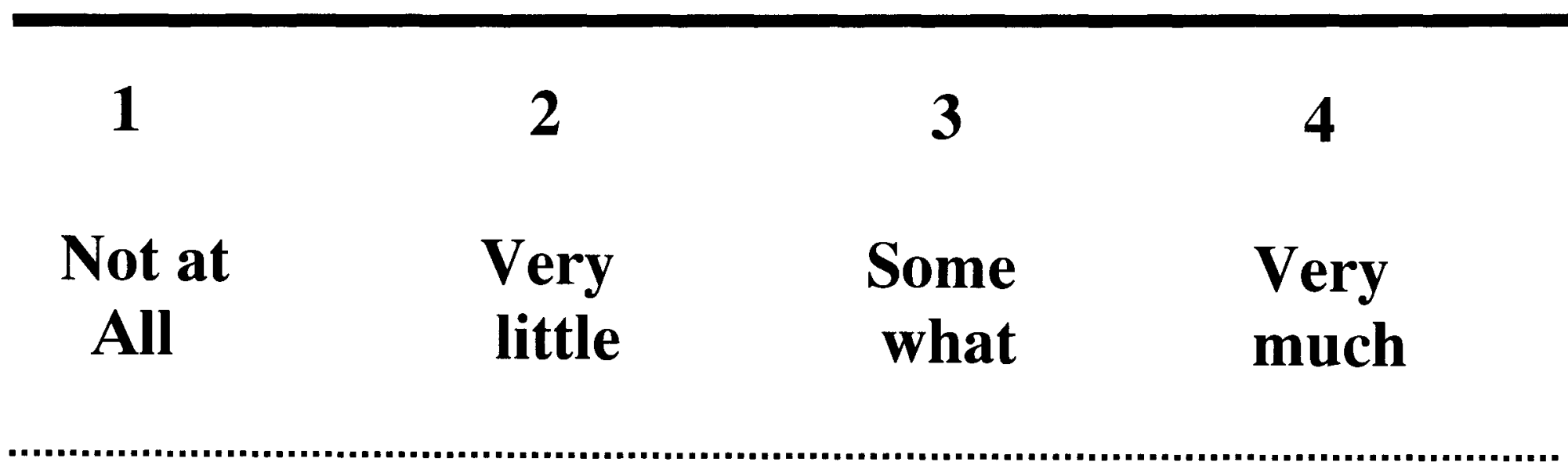




\section{Proactive Coping Inventory (PCI)}

1

Not at all true

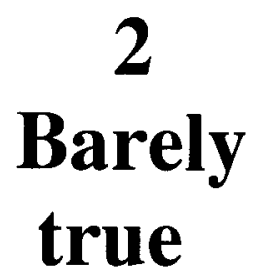

true

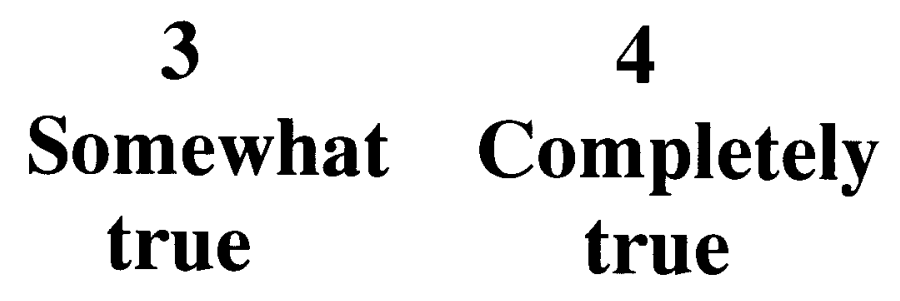

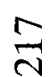


Appendix L

Laminated examples of Likert scales, continued

\section{Successful Aging Inventory (SAI)}

\begin{tabular}{ccccc}
\hline 0 & 1 & 2 & 3 & 4 \\
$\begin{array}{c}\text { Hardly } \\
\text { ever }\end{array}$ & Sometimes & $\begin{array}{c}\text { About half } \\
\text { the time }\end{array}$ & $\begin{array}{c}\text { Most of } \\
\text { the time }\end{array}$ & $\begin{array}{c}\text { Almost } \\
\text { always }\end{array}$
\end{tabular}


Appendix M

Individual Record of Gift Card Receipt

I verify that I have received a $\$ 5$ Gift Card to the Market on the ground floor of Chapel House as compensation for participating in the research study.

Signature

Date

Thank you very much for participating!

Page 1 of 1 
Appendix N

Study Rosters Included ___ Excluded

\begin{tabular}{|l|l|l|}
\hline $\begin{array}{c}\text { Unique } \\
\text { Identifier }\end{array}$ & Participant Name & $\begin{array}{c}\text { Data Collection } \\
\text { Session Date \& Time }\end{array}$ \\
\hline & & \\
\hline & & \\
\hline & & \\
\hline & & \\
\hline & & \\
\hline & & \\
\hline & & \\
\hline & & \\
\hline & & \\
\hline & & \\
\hline & & \\
\hline & & \\
\hline & & \\
\hline & & \\
\hline & & \\
\hline & & \\
\hline & & \\
\hline & & \\
\hline & & \\
\hline & & \\
\hline & & \\
\hline & & \\
\hline & & \\
\hline & & \\
\hline & & \\
\hline & & \\
\hline & & \\
\hline & & \\
\hline & & \\
\hline & & \\
\hline & & \\
\hline & & \\
\hline & & \\
\hline & & \\
\hline & & \\
\hline & & \\
\hline & & \\
\hline & & \\
\hline & & \\
\hline & & \\
\hline & & \\
\hline & & \\
\hline & & \\
\hline & & \\
\hline & & \\
\hline & & \\
\hline
\end{tabular}

Page 1 of 1 
Appendix $\mathrm{O}$

Letter of Agreement with Study Site

Letter of Support

April 15, 2009

To Whom It May Concern:

This letter is written in support of the research study, Exploring a New Theory of Successful Aging for Usefulness among Low-Income Older Adults in an Independent and Assisted Living Retirement Community, to be conducted at the Christian Care Community (CCC) in Louisville, Kentucky. The CCC is a non-profit, church-funded continuing care retirement community serving low-income older adults. To our knowledge, there are few, if any, other retirement facilities in Kentucky offering assisted living services for older adults without the financial resources necessary to cover the costs of assisted living care.

The CCC will support the research study by providing a list of resident names, telephone numbers, apartment numbers, and rent categories, unless a resident requests to be excluded. We understand about 150 residents in independent or assisted living apartments will be randomly selected, contacted by telephone, and asked to voluntarily participate in the study. The CCC will provide a private meeting space with chairs and tables to seat 5 to 7 people, allowing space between individuals so that answers on written questionnaires may remain confidential.

We will also assure that a staff member is available at the beginning of each meeting to assist, according the CCC policies and procedures, any resident who may experience dementia which causes disruptive or distracting behavior. It is understood that the investigator will notify social services staff if this occurs or if the results of dementia screening indicate abnormal cognitive function, to provide for resident safety and wellbeing.

We understand that all resident's responses will be confidential and only aggregated data will be shared with the CCC. We feel the information obtained in this study may increase understanding of how our residents adjust to changes experienced as they age, and how they feel about growing older. This knowledge may be useful to guide the care and services we offer our residents, and potentially may influence public policy and program development in future. Thus CCC is pleased to offer support for the University of Louisville School of Nursing investigator to conduct this study.

Yours,

Jane Mobley

Director of Social Services 
Appendix $\mathbf{P}$

Institutional Review Board Approvals

LETTER OF APPROVAL OF EXEMPT STATUS

and

LETTER OF APPROVAL OF ADDENDUM TO PROTOCOL 


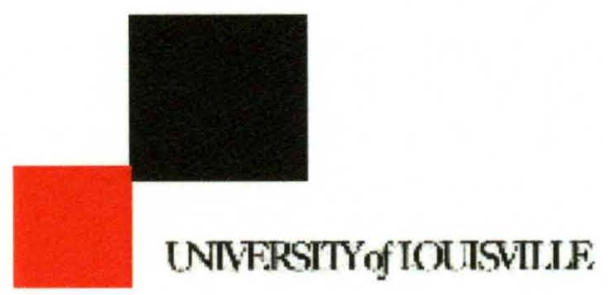

INSTITUTIONAL REVIEW BOARDS

University of Loulsville

MedCenter One, sulte 200

501 E. Broadway

Loulsville, Kentuciky 40202-1798

Once: $\quad 502-852-5188$

Fax: $\quad 502-852-2164$

\author{
To: $\quad$ Speck, Barbara \\ From: $\quad$ The University of Louisville Institutional Review Board (IRB) \\ Date: $\quad$ Thursday, April 23, 2009 \\ Subject: IRB Correspondence \\ Tracking \#: 09.0209 \\ Title: $\quad$ Exploring a New Theory of Successful Aging among Low-Income \\ Older Adults in an Independent and Assisted Living Retirement \\ Community
}

This study was reviewed on $4 / 22 / 2009$ and determined by the chair of the Institutional Review Board that the study is exempt according to 45 CFR 46.101(b) 2 since (2) Research involving the use of educational tests (cognitive, diagnostic, aptitude, achievement), survey procedures, interview procedures or observation of public behavior, unless: (i) information obtained is recorded in such a manner that human subjects can be identified, directly or through identifiers linked to the subjects; and (ii) any disclosure of the human subjects' responses outside the research could reasonably place the subjects at risk of criminal or civil liability or be damaging to the subjects' financial standing, employability, or reputation.. The study is exempt only if information that could identify subjects is not recorded.

Since this study has been found to be exempt, no additional reporting, such as submission of Progress Reports for continuation reviews, is needed. If your research focus or activities change, please submit a Study Amendment Request Form to the IRB for review to ensure that the study still meets exempt status. Best wishes for a successful study. Please send all inquires and electronic revised/requested items to our office email address at hsppofc-louisville.edu. 


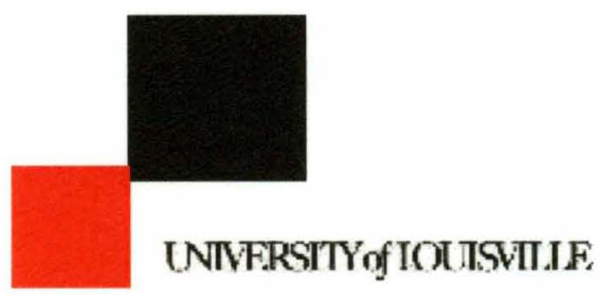

INSTITUTIONAL REVIEW BOARDS

University af Loulsville

MedCenter One, sute 200

$501 \mathrm{E}$. Broadway

Loulsville, Kentucky 40202-1796

Once: $\quad 502-852-5188$

Fax: $\quad 502-852-2164$

To: $\quad$ Speck, Barbara

From: The University of Louisville Institutional Review Board (IRB)

Date: $\quad$ Friday, May 15, 2009

Subject: Approval Letter

Tracking \#: AMEND-1235 (09.0209)

Title: $\quad$ Exploring a New Theory of Successful Aging among Low-Income Older Adults in an Independent and Assisted Living Retirement Community

Determination Date: 05/14/2009

The following items have been received by the Human Subjects Protection Program Office and were reviewed by the chair/vice-chair of the Institutional Review Board (IRB):

- Amend-1235, dated 05/13/2009

The modifications include:

- The time when WHEN the Informed Consent and HIPAA documents are provided to participants has been modified in hopes to have more successful recruitment of subjects.

This information has been reviewed and determined by the chair of the Institutional Review Board (IRB) not to change the exempt status of this study.

Thank you for keeping us informed of your study.

Thank you.

Pite $m$ dusach 


\title{
CURRICULUM VITA
}

\author{
Valerie L. McCarthy, BSN, RN
}

3504 Middle Road Jeffersonville, In. 47130

Phone: 812-282-0670 Cell: 502-819-5037 vemcca01@louisville.edu

Education

2005 - present Candidate, Doctor of Philosophy in Nursing BSN/PhD Program School of Nursing, University of Louisville, Louisville, Kentucky G.P.A. 3.92

Anticipated completion date: Summer, 2009

$2005-2006$

Masters of Science in Nursing, Core Curriculum (30 hours)

University of Louisville School of Nursing, Louisville, Kentucky

1999

Bachelors of Science in Nursing

School of Nursing, Indiana University, New Albany, Indiana

1972

Associate of Science in Nursing

School of Nursing, Indiana University, New Albany, Indiana

\section{Certifications \& Licensure}

Kentucky Registered Nurse, License 1028917

Indiana Registered Nurse, License 28055560A

Indiana State Department of Health Qualified Medication Aide Instructor, 2003 - 2006 Indiana State Department of Health Certified Nurse Aide Instructor, 1993 - 2006

American Lung Association Certified Instructor for TB-PPD Administration, 2001-2005 


\section{Professional Employment}

2007-2008 Graduate Research Assistant

University of Louisville School of Nursing

2006-2007 Graduate Teaching Assistant

University of Louisville School of Nursing

2005-2006 Graduate Research Assistant

University of Louisville School of Nursing

1999- 2006 Owner and Chief Executive Officer

Healthcare Education and Training Consultants, Inc., Clarksville, In.

1997-1999 Adjunct Faculty, LPN Program

Ivy Tech State College, Sellersburg, In.

1998 - 1999 Director of Staff Development

Transitional Health Services Of Clark County, Jeffersonville, In.

1995 - 1998 Director of Staff Development

Willowbrook Healthcare, Clarksville, In.

1993 - 1995 Staff Development Coordinator

GreenValley Health and Rehabilitation Center, New Albany, In.

1991 - 1993 Direct, Extended Care Division

Visiting Nurse Association of Southeastern Indiana, Inc.,

Jeffersonville, In.

1987 - 1990 Director of Nursing, Companion Care Division

Visiting Nurse Association of Louisville, Inc., Louisville, Ky.

1985 - 1987 Clinical Nursing Supervisor

Upjohn Home Healthcare, Louisville, Ky.

1976 - 1985 Critical Care Staff Nurse

Methodist Hospital, Louisville, Ky.

1972 - 1975 Staff Nurse, Neonatal ICU, Pediatric Open Heart unit Kosair Children's Hospital, Louisville, Ky. 


\section{$\underline{\text { Scholarship }}$}

Grants: Training

Ruth Craddock Graduate Student Research Award, 2008. Funded by the School of Nursing, University of Louisville. (\$600)

University of Louisville Graduate Fellowship to support dissertation research on the role of adaptation and transcendence in successful aging among older adults in an assisted living and independent community, 2008-9. Funded by the Graduate School, University of Louisville. ( $\$ 20,000$ stipend, tuition and insurance)

Ruth Craddock Graduate Student Research Award, 2007. Funded by the School of Nursing, University of Louisville. (\$500)

Graduate Assistantship, 2005 - 2008. Funded by University of Louisville, Louisville, Kentucky. ( $\$ 20,000$ stipend, tuition and insurance)

\section{Grants: Research}

Sigma Theta Tau Dissertation Support, 2009. Funded by the Iota Zeta Chapter. (\$500)

Research Assistant. 2005-2006, 2007-2008. University of Louisville School of Nursing

Research Assistant and Co-investigator. 2007 - present. A Survey of Physical Activity Counseling among Kentucky Advance Practice Nurses. Dr. Barbara Speck, PI. Intramural funding, $\$ 5000$.

Research Assistant, 2005 - 2006. Study of Primary Health Care among Clients of the Harambee Nurse-managed Health Center Based at the Presbyterian Community Center, Louisville, KY. Dr. Kay Roberts, PI. Unfunded.

Member, Indiana Governor's Task Force. Participated in survey design and data collection, 1998. Survey of Long-Term Care and Area Agency on Aging Service Providers Regarding Attitudes toward Abuse \& Neglect. Funded by Indiana Family and Social Services Administration.

Consultant with Visiting Nurse Association of Louisville. Designed and coordinated data collection, budget, and participated in writing of study report, 1988. Survey of Perceived Needs of Elderly Residents of Jefferson County, Kentucky and the Role of Family, Church, and Neighbors in Meeting Needs. Funded by Kentuckiana Regional Planning and Development Administration (KIPDA). (\$12,000). 


\section{$\underline{\text { Publications }}$}

McCarthy, V. L. \& Freeman, L. H. (2008). A multidisciplinary concept analysis of empowerment: Implications for nursing. Journal of Theory Construction and Testing, $12(2), 68-74$.

McCarthy, V. L. (2003). Mound Builders: Mythic Lost Race or Native American Indians? Currents of Change: Journal of the Falls of the Ohio Archaeology Society, 1(1), 63-71.

\section{$\underline{\text { Presentations }}$}

\section{Podium Presentations}

Speck, B. J., McCarthy, V. L., \& Myers, J. (February 2009). Survey of Physical Activity Assessment and Counseling Practices of Kentucky Advanced Registered Nurse Practitioners. Southern Nursing Research Society Annual Conference.

Indiana University Annual Undergraduate Research Symposium: Mound Builders: Mythic Lost Race or Native American Indians? Indianapolis, In. 1999.

Presentation on Pilot Program for Training Modules I and II on Prevention of Abuse \& Neglect, at Indiana University for Indiana Governor's Task Force on Prevention of Abuse and Neglect. New Albany, Indiana. 1999.

Community Health Care and Home Care Services Lecture Series for Southern Indiana Council on Aging and Aged, Area XV. New Albany, Indiana. 1993-94.

Proposed Changes to Indiana's CHOICE Homecare Funding Program, to the Liaison Committee, Department of Aging and Rehabilitation Services, Indianapolis, In. 1993.

Program for 105-Hour Certified Nursing Assistant Training, for a consortium of eleven home health service providers in Louisville, Ky. 1990.

\section{Poster Presentation}

Speck, B. J., McCarthy, V. L., \& Myers, J. (October, 2008). Survey of Physical Activity Assessment and Counseling Practices of Kentucky Advanced Registered Nurse Practitioners. $136^{\text {th }}$ American Public Health Association Annual Meeting \& Exposition. San Diego, CA.

\section{Doctoral Practicum Experience}

Invited to assist Dr. Karen Robinson, Inaugural American Academy of Nursing - AARP Joint Fellow at the AARP Public Policy Institute in Washington, D.C. during two weeks in March, 2008. Completed an accepted draft of a strategic plan for the Robert Wood Johnson - AARP Center to Champion Nursing; attended the American College of Nurse 
Practitioners' Advanced Public Policy Institute for Health Professionals; attended executive board meeting, American Academy of Nursing; co-authored an OpEd piece subsequently published in USA Today.

\section{Professional Awards, Honors, and Scholarships}

Nominated University of Louisville Faculty Favorite, January, 2009

University of Louisville Graduate Fellowship, August, 2008

Ruth Craddock Scholarship Recipient, 2007 and 2008

Sigma Theta Tau Honor Society of Nursing Dissertation Support, 2007

Graduate Assistantships, 2005 - 2008. University of Louisville, Louisville, Kentucky.

Outstanding Student, Indiana University Southeast School of Nursing, 1999.

New Albany, Indiana.

Professional Organizations

\section{Current}

American Geriatric Society

American Nurses Association

American Public Health Association

Gerontological Nursing Association

Indiana Nurses Association

Kentucky Nurses Association

Southern Nursing Research Society

The Gerontological Society of America

Past

Indiana Association of Homes \& Services of the Aging

Indiana Home Healthcare Association

Kentucky Home Healthcare Association

$\underline{\text { Service }}$

\section{Professional Service}

Diabetes education program for residents, staff, and administration at Christian Care Communities, Louisville, KY. Summer, 2007.

Served on the Indiana Governor's Task Force on Prevention of Abuse and Neglect. New Albany, Indiana. Indianapolis, In. 1998-1999.

Served on the Indiana State Department of Health - Family and Social Services Administration Liaison Committee. Indianapolis, In. 1996 - 1998

Provided a series of lectures on Community Health Care and Home Care Services for the Southern Indiana Council on Aging and Aged, Area XV. New Albany, Indiana. 1993/94. 
Served on the Liaison Committee, Department of Aging and Rehabilitation Services, for the Indiana CHOICE Homecare Funding Program. Indianapolis, In. 1993.

Served on the Kentucky Home Health Association Homecare Committee. Frankfort, Kentucky. 1988-1990.

\section{Community Service}

Clark County Indiana Democratic Party, 2000 - present.

Election volunteer for Democratic Primary candidate for Jeffersonville City Court Judge, 2007 and for Democratic Primary Presidential candidate, 2007-2008.

Harambee Nursing Center Health Fair at Presbyterian Community Center.

\section{School of Nursing Service}

Student Representative, Graduate Academic Affairs Committee, University of Louisville, Louisville, KY. 2008-2009

Wrote and assisted with implementation of diversity program, HIPAA compliance, and agency policies and procedures to achieve compliance with Medicaid/Pass Port funding standards for the Harambee Nurse-Managed Health Center at the Presbyterian Community Center in Louisville, Kentucky. 2005-2006.

\section{Teaching Experience}

\section{Currently Enrolled}

Health professions education certification program. University of Louisville. 2008/2009.

\section{Undergraduate}

Graduate Teaching Assistant. Community Health Nursing Clinical Rotation (C341), University of Louisville School of Nursing. 2006/7.

\section{Licensed Practical Nursing Program}

Clinical Instructor. Adult Medical Surgical Nursing Clinical Rotation, IVY Tech Vocational and Technical College, Licensed Practical Nursing Program. 1996 - 1997.

\section{State and Federal Certification Programs}

Instructor. Indiana State Department of Health 100-Hour Qualified Medication Administration Course, Healthcare Education \& Training Consultants, Inc. Clarksville, IN. $2003-2006$.

Instructor. Indiana State Department of Health 130-Hour Certified Nurse Aide Training Program for Healthcare Education \& Training Consultants, Inc. Clarksville, IN. 1993 2006. 


\section{Consultant Activities}

Compliance with Indiana Family and Social Service Administration regulations on Medication Administration and Safety for Service Providers for the Developmentally Delayed. Rauch Inc. New Albany, Indiana. 2003 and 2005.

Compliance with Indiana State Department of Health, Division of Long Term Care and Department of Health and Human Services, Division of Medicare standards for staff development and training. Transitional Health Care Center of Clark County, Jeffersonville, Indiana. 1998.

Team Building and Group Dynamics Workshop for Facility Managers. GranCare Healthcare, Inc. Clarksville, Indiana. 1996. 